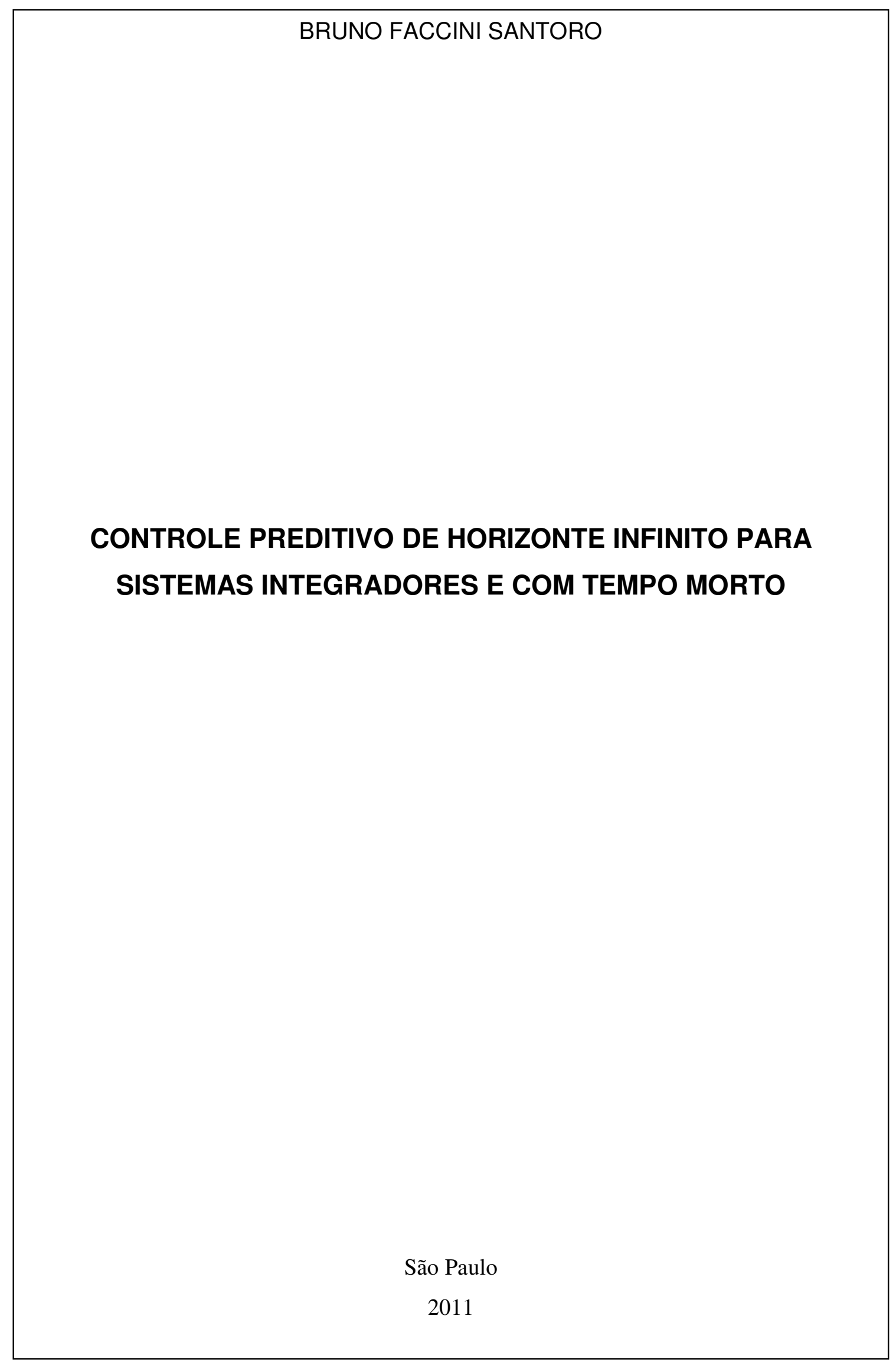




\title{
CONTROLE PREDITIVO DE HORIZONTE INFINITO PARA SISTEMAS INTEGRADORES E COM TEMPO MORTO
}

\author{
Dissertação apresentada à Escola \\ Politécnica da Universidade de São \\ Paulo para obtenção do título de Mestre \\ em Engenharia
}

São Paulo 


\title{
BRUNO FACCINI SANTORO
}

\section{CONTROLE PREDITIVO DE HORIZONTE INFINITO PARA SISTEMAS INTEGRADORES E COM TEMPO MORTO}

\author{
Dissertação apresentada à Escola \\ Politécnica da Universidade de São \\ Paulo para obtenção do título de Mestre \\ em Engenharia \\ Área de concentração: Engenharia \\ Química
}

Orientador: Prof. Dr. Darci Odloak

São Paulo 
Este exemplar foi revisado e alterado em relação à versão original sob responsabilidade única do autor e com anuência de seu orientador

São Paulo, 14 de março de 2011

Bruno Faccini Santoro (autor)

Prof. Dr. Darci Odloak (orientador)

FICHA CATALOGRÁFICA - EDIÇÃO REVISADA

\section{Santoro, Bruno Faccini}

Controle preditivo de horizonte infinito para sistemas inte gradores e com tempo morto / B.F. Santoro. -- São Paulo, 2011. $112 \mathrm{p}$.

Dissertação (Mestrado) - Escola Politécnica da Universidade de São Paulo. Departamento de Engenharia Química.

1. Controle preditivo 2. Controle de processos 3. Observadores de estado I. Universidade de São Paulo. Escola Politécnica. Departamento de Engenharia Química II. t. 


\section{AGRADECIMENTOS}

Ao Prof. Dr. Darci Odloak, pelo apoio, compreensão e paciência durante a elaboração desta dissertação.

Aos membros da banca de qualificação, pelas sugestões de melhoria e correções.

Aos meus pais, por terem me preparado para os desafios da vida.

À minha avó, pelos ensinamentos.

À minha irmã, que não entendeu o que é tempo morto mas me apoiou mesmo assim.

À minha namorada, pelo incentivo e companhia.

À Op2B, em especial aos engenheiros Maurício, Zeca e Mário, pelas diversas lições de trabalho em grupo, liderança e até mesmo otimização.

Ao Tiago, pelas respostas pertinentes às dúvidas mais inesperadas.

Ao Klauss e à Aline, pelas boas risadas.

A todos os amigos do laboratório, pelo ambiente agradável. 
“(...) e pensa em como seria bom se a gente pudesse voltar atrás e corrigir todas as escolhas erradas que fez na vida, mas como saber se a escolha era errada ou não, já que a vida não tem gabarito?"

(Luís Fernando Verissimo) 


\section{RESUMO}

Controle preditivo baseado em modelo (MPC) recebeu ampla aceitação na indústria química nos últimos 30 anos. O funcionamento básico dessa técnica é a utilização de um modelo para calcular o comportamento de uma planta em função das entradas que ela receberia nos próximos instantes. Define-se um objetivo, cuja principal contribuição é dada por uma medida da distância entre a condição predita da planta e um valor desejado previamente estipulado. Esse objetivo pode incluir ainda, por exemplo, penalizações sobre o esforço de controle necessário para levar a planta a uma condição mais próxima do desejável. São incorporadas restrições como limites físicos da planta e dos atuadores e formula-se um problema de otimização, buscando o ponto ótimo dessa função objetivo e respeitando as restrições.

Neste trabalho é abordado o problema de controle preditivo baseado em modelo para sistemas que apresentem integradores e/ou tempos mortos. Estes elementos tornam mais difícil o controle de processos baseado apenas em técnicas clássicas. Apresenta-se aqui um modelo em espaço de estados que permite a representação dessas dinâmicas de modo suficientemente preciso. A formulação de modelo apresentada permite ainda a incorporação de informações sobre distúrbios medidos. É feita uma demonstração da estabilidade desse controlador quando o modelo por ele utilizado é idêntico ao comportamento real da planta.

Numa aplicação real do controlador proposto, seria necessário estimar os estados da planta a partir das medidas das saídas. Em geral, utiliza-se um Filtro de Kalman para realizar esta tarefa. São estudados aqui os efeitos que a presença desse filtro teria sobre o desempenho do sistema em malha fechada. É proposto um observador baseado numa mudança heurística feita sobre o Filtro de Kalman e que permite, em certos casos, uma melhoria de desempenho. São apresentados os resultados de simulações de uma planta de óxido de etileno com o intuito de ilustrar a atuação do controlador estável desenvolvido e do observador proposto.

Palavras-chave: Controle Preditivo com Modelo, Processos com tempo morto, Sistemas integradores 


\begin{abstract}
Model Predictive Control (MPC) has gained wide acceptance in chemical industry in the last 30 years. The basic principle of this technique is to use a model to calculate plant's future behavior based on the inputs it would receive in the next sampling periods. It must be set an objective, mainly composed of some measure of the distance between plant's predicted state and a previously specified condition. Objective value may also include, for example, penalty on control effort necessary to drive the plant closer to the desired state. It is possible to include constraints, such as physical limits of the plant or of the actuators and therefore to pose an optimization problem, searching the best value of the objective function that satisfies all constraints.

This work addresses the problem of MPC applied to integrating systems and/or processes with dead-time. These kinds of plants are often difficult to control using only classical techniques. It is presented here a state space model to represent both cases accurately. Measured disturbances may also be incorporated to the model. Finally, it is shown that the proposed controller is stable when its internal model represents exactly plant's dynamics.

In any real application of this controller, it would be necessary to estimate plant's states from outputs' measures. In general, Kalman Filter solves this problem. It is studied in this work the effects caused by filter's inclusion on closed loop performance. A new observer is proposed, based on a heuristic improvement over Kalman Filter which induces, for some systems, improved performance. Numerical simulation has been performed over a model of an ethylene oxide plant, illustrating the use of this stable controller and the proposed observer.
\end{abstract}

Keywords: Model Predictive Control, Processes with dead-time, Integrating systems 


\section{LISTA DE ILUSTRAÇÕES}

Figura 2-1: Estrutura de controle em camadas, Zanin (2001) .......................................... 33

Figura 6-1: Visão geral de uma planta de óxido de etileno - Carrapiço (2004) ..................... 83

Figura 6-2: Evolução das saídas do sistema - sem target nas entradas .................................87 87

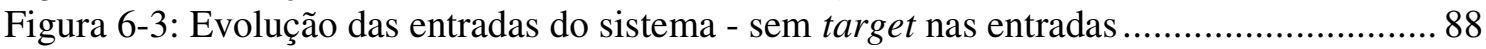

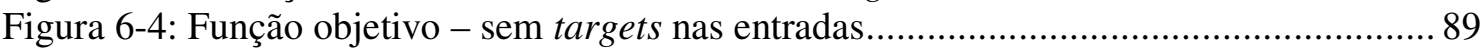

Figura 6-5: Evolução das saídas do sistema - com target nas entradas ................................ 91

Figura 6-6: Evolução das entradas do sistema - com target nas entradas............................. 92

Figura 6-7: Função objetivo - com targets nas entradas .............................................. 92

Figura 6-8: Evolução das entradas do sistema com medição do estado ou Filtro de Kalman 94

Figura 6-9: Evolução das saídas do sistema com medição do estado ou Filtro de Kalman..... 95

Figura 6-10: Evolução da função objetivo com medição do estado ou Filtro de Kalman....... 96

Figura 6-11: Comparação entre os autovalores das matrizes de transição dos erros de

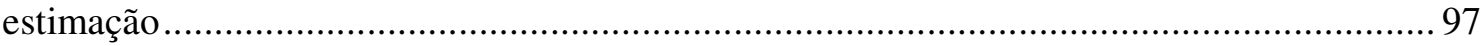

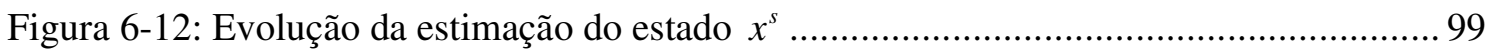

Figura 6-13 : Evolução das saídas quando o estado inicial é desconhecido ......................... 100

Figura 6-14 : Evolução das entradas quando o estado inicial é desconhecido...................... 101

Figura 6-15 : Evolução da função objetivo quando modelo o estado inicial é desconhecido 102

Figura 6-16 : Evolução das saídas quando modelo tem incerteza nas constantes de tempo.. 104

Figura 6-17 : Evolução das entradas quando modelo tem incerteza nas constantes de tempo

Figura 6-18 : Evolução da função objetivo quando modelo tem incerteza nas constantes de tempo. 


\section{LISTA DE TABELAS}

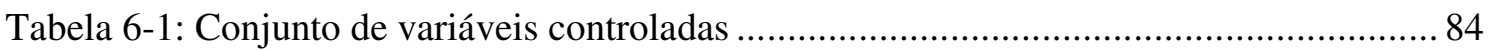

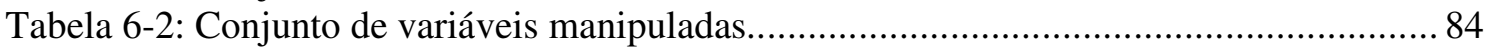

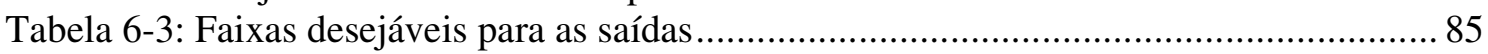

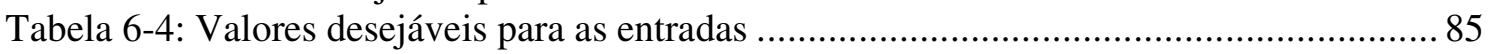

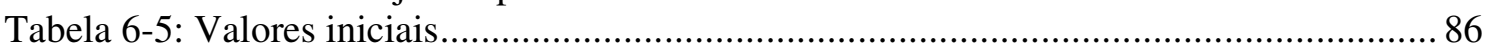

Tabela 6-6: Sintonia utilizada sem considerar targets nas entradas .................................. 86

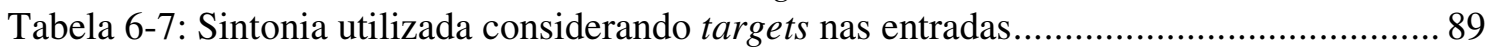

Tabela 6-8: Sintonia utilizada considerando medição completa do estado........................... 93 


\section{LISTA DE ABREVIATURAS E SIGLAS}

$\begin{array}{ll}\text { BMI } & \text { Bilinear Matrix Inequality } \\ \text { DMC } & \text { Dynamic Matrix Control } \\ \text { FCC } & \text { Fluid Catalytic Cracking } \\ \text { GAMS } & \text { General Algebraic Modelling System } \\ \text { IHMPC } & \text { Infinite Horizon Model Predictive Control } \\ \text { LMI } & \text { Linear Matrix Inequality } \\ \text { MATLAB } & \text { Matrix Laboratory } \\ \text { MIMO } & \text { Multiple Input, Multiple Output } \\ \text { MPC } & \text { Model Predictive Control } \\ \text { PNL } & \text { Programação não-linear } \\ \text { QP } & \text { Quadratic Programming } \\ \text { SDCD } & \text { Sistema digital de controle distribuído } \\ \text { SISO } & \text { Single Input, Single Output }\end{array}$




\section{LISTA DE SÍMBOLOS}

\begin{tabular}{|c|c|}
\hline 0 & Matriz nula de dimensão qualquer \\
\hline$a$ & Constante utilizada na demonstração de estabilidade \\
\hline$A$ & Matriz de transição dos estados \\
\hline$A_{X X}, A_{X Z}, A_{Z Z}$ & Matrizes da dinâmica do sistema quando particionado em $X$ e $Z$ \\
\hline $\bar{A}$ & Matriz utilizada no cálculo da predição das saídas \\
\hline$B$ & Matriz que relaciona as entradas aos estados do sistema \\
\hline $\bar{B}$ & Matriz utilizada no cálculo da predição das saídas \\
\hline$b$ & (seção 5) Constante utilizada na demonstração de estabilidade \\
\hline$b$ & $\begin{array}{l}\text { (demais seções) Parte real dos pólos complexos conjugados de uma } \\
\text { função de transferência }\end{array}$ \\
\hline$B_{l}^{d}$ & Matriz que relaciona as ações de controle à componente $x^{d}$ \\
\hline$B_{l}^{i}\left(B w_{l}^{i}\right)$ & $\begin{array}{l}\text { Matriz que relaciona as ações de controle (resp. distúrbios medidos) } \\
\text { à componente } x^{i}\end{array}$ \\
\hline$B_{l}^{S}\left(B w_{l}^{s}\right)$ & $\begin{array}{l}\text { Matriz que relaciona as ações de controle (resp. distúrbios medidos) } \\
\text { à componente } x^{s}\end{array}$ \\
\hline$B w_{l}^{p}$ & Matriz que relaciona os distúrbios medidos à componente $x^{w}$ \\
\hline$C$ & Matriz que relaciona os estados às saídas do sistema \\
\hline $\bar{C}$ & $\begin{array}{l}\text { Bloco da matriz } C \text { que corresponde apenas às saídas do sistema, não } \\
\text { aos estados } z\end{array}$ \\
\hline$c_{2 b}$ & Termo constante da função objetivo do problema $\mathrm{P} 2 \mathrm{~b}$ \\
\hline Co & $\begin{array}{l}\text { Matriz usada na predição dos estados futuros, semelhante à matriz de } \\
\text { controlabilidade }\end{array}$ \\
\hline$C_{y X}$ & Matriz que relaciona o bloco $X$ às saídas $y$ \\
\hline$d_{i, j}^{0}\left(d w_{i, j}^{0}\right)$ & Ganho da função de transferência $G_{i, j}\left(\right.$ resp. $\left.G_{i, j}^{w}\right)$ \\
\hline$d_{i, j, k}^{d}\left(d w_{i, j, k}^{p}\right)$ & k-ésimo resíduo da função de transferência $G_{i, j}\left(\operatorname{resp} . G_{i, j}^{w}\right)$ \\
\hline$d_{i, j}^{i}\left(d w_{i, j}^{i}\right)$ & Incremento na função de transferência integradora $G_{i, j}\left(\right.$ resp. $\left.G_{i, j}^{w}\right)$ \\
\hline$D^{d}$ & Matriz que concentra todos os $d_{i, j, k}^{d}$ \\
\hline
\end{tabular}




\begin{tabular}{|c|c|}
\hline$D w^{p}$ & Matriz que concentra todos os $d w_{i, j, k}^{p}$ \\
\hline$e$ & Erro entre o estado real e o estimado \\
\hline$E[\cdot]$ & Operador esperança \\
\hline$f_{2 b}$ & Vetor do termo linear da função objetivo do problema P2b \\
\hline$F$ & Matriz com a dinâmica dos modos estáveis \\
\hline$F_{i}$ & Matrizes simétricas utilizadas na definição de LMIs \\
\hline$F^{w}$ & $\begin{array}{l}\text { Matriz com a dinâmica dos modos estáveis das relações distúrbio- } \\
\text { saída }\end{array}$ \\
\hline$G(s)$ & Função de transferência que representa o sistema a ser controlado \\
\hline$G_{j}$ & Matrizes simétricas utilizadas na definição de BMIs \\
\hline$H_{2 b}$ & Matriz do termo quadrático da função objetivo do problema P2b \\
\hline$H_{i j}$ & Matrizes simétricas utilizadas na definição de BMIs \\
\hline$H c$ & Horizonte de controle \\
\hline$H p$ & Horizonte de predição \\
\hline$I_{n}$ & Matriz identidade de dimensão $n$ \\
\hline$I_{n y}^{*}$ & $\begin{array}{l}\text { Matriz identidade modificada, em que as componentes das saídas não } \\
\text { integradoras são iguais a } 0\end{array}$ \\
\hline $\bar{I}_{n u}$ & Matriz auxiliar utilizada nas restrições de limite sobre as entradas \\
\hline $\bar{I}_{n y}$ & $\begin{array}{l}\text { Matriz auxiliar utilizada no cálculo da contribuição à função objetivo } \\
\text { dada pelo desvio entre as saídas e as zonas desejadas }\end{array}$ \\
\hline$J$ & $\begin{array}{l}\text { Matriz auxiliar para realizar a correspondência entre as ações de } \\
\text { controle e as componentes de } B_{l}^{d}\end{array}$ \\
\hline$J^{w}$ & $\begin{array}{l}\text { Matriz auxiliar para realizar a correspondência entre os distúrbios } \\
\text { medidos e as componentes de } B w_{l}^{p}\end{array}$ \\
\hline$k$ & Instante atual \\
\hline$K_{i, j}$ & Ganho da função de transferência $G_{i, j}(s)$ \\
\hline$K_{K}$ & Ganho do Filtro de Kalman \\
\hline$K_{F}$ & Ganho do observador heurístico proposto \\
\hline$L$ & Ganho do observador \\
\hline
\end{tabular}




\begin{tabular}{|c|c|}
\hline$m$ & Horizonte de controle \\
\hline $\bar{M}$ & Matriz auxiliar utilizada nas restrições de limites sobre as entradas \\
\hline$N$ & $\begin{array}{l}\text { (seção 2) Contribuição do ruído de processo ao estado } x \\
\text { (demais seções) Matriz auxiliar para realizar a correspondência entre }\end{array}$ \\
\hline & as ações de controle e as componentes de $B_{l}^{d}$ \\
\hline$N^{w}$ & $\begin{array}{l}\text { Matriz auxiliar para realizar a correspondência entre os distúrbios } \\
\text { medidos e as componentes de } B w_{l}^{p}\end{array}$ \\
\hline$n a$ & Ordem das funções de transferência $G_{i, j}(s)$ \\
\hline$n d$ & Dimensão da componente $x^{d}(n d=n u \cdot n y \cdot n a)$ \\
\hline$N_{d}\left(N_{i}, N_{y}\right)$ & Matriz utilizada para extrair a componente $x^{d}\left(\right.$ resp. $\left.x^{i}, x^{s}\right)$ \\
\hline$n i$ & Número de saídas integradoras \\
\hline$n p$ & Dimensão da componente $x^{w}(n p=n w \cdot n y \cdot n a)$ \\
\hline nu & Número de entradas \\
\hline$n w$ & Número de distúrbios medidos \\
\hline ny & Número de saídas \\
\hline $\mathfrak{O}$ & Matriz de observabilidade \\
\hline$P$ & Matriz de covariância do erro de estimação \\
\hline$P_{F}$ & $\begin{array}{l}\text { Matriz simétrica positiva definida calculada no algoritmo do filtro } \\
\text { heurístico }\end{array}$ \\
\hline$Q$ & Peso da função objetivo (formulação DMC) \\
\hline $\bar{Q}$ & Matriz utilizada no cálculo do custo terminal no problema P2b \\
\hline$Q_{i}$ & $\begin{array}{l}\text { Peso na função objetivo do problema } \mathrm{P} 2 \mathrm{a} \text { sobre os modos } \\
\text { integradores }\end{array}$ \\
\hline$Q_{u}$ & $\begin{array}{l}\text { Peso na função objetivo sobre a distância entre as entradas e o target } \\
\text { desejado }\end{array}$ \\
\hline $\bar{Q}_{u}$ & Extensão da matriz $Q_{u}$ para considerar todo o horizonte de controle \\
\hline$Q_{y}$ & $\begin{array}{l}\text { Peso na função objetivo sobre a distância entre as saídas e a zona } \\
\text { desejada }\end{array}$ \\
\hline $\bar{Q}_{y}$ & Extensão da matriz $Q_{y}$ para considerar todo o horizonte de controle \\
\hline$r_{i, j, k}$ & k-ésimo pólo da função de transferência $G_{i, j}(s)$ \\
\hline
\end{tabular}




\begin{tabular}{|c|c|}
\hline$R$ & Peso na função objetivo sobre as ações de controle \\
\hline $\bar{R}$ & Extensão da matriz $R$ para considerar todo o horizonte de controle \\
\hline $\bar{R}^{\prime}$ & Peso na função objetivo do problema P2a sobre as ações de controle \\
\hline$R_{1}, R_{2}, R_{12}$ & Matrizes de covariância dos ruídos \\
\hline$S$ & Peso da função objetivo (formulação DMC) \\
\hline$S_{i, j}(s)$ & Resposta ao degrau da função de transferência $G_{i, j}(s)$ \\
\hline$S_{i}, S_{u}, S_{y}$ & Pesos na função objetivo sobre as variáveis de folga $\delta_{i, k}, \delta_{u, k}, \delta_{y, k}$ \\
\hline$t$ & Tempo \\
\hline$T_{1}, \cdots, T_{5}$ & Termos da função objetivo do problema $\mathrm{P} 2 \mathrm{~b}$ \\
\hline$u$ & Vetor de entradas do sistema \\
\hline$U(t)$ & Função degrau \\
\hline$u^{a}$ & Vetor de entradas do sistema no problema $\mathrm{P} 2 \mathrm{a}$ \\
\hline$u^{b}$ & Vetor de entradas do sistema no problema $\mathrm{P} 2 \mathrm{~b}$ \\
\hline$u_{\text {des }}$ & Valor desejado (target) para as entradas do sistema \\
\hline$u_{\max }$ & Limite superior do valor das entradas do sistema \\
\hline$u_{\min }$ & Limite inferior do valor das entradas do sistema \\
\hline$v_{1}$ & Ruído de processo \\
\hline$v_{2}$ & Ruído de medida \\
\hline$V_{k}$ & Custo total (função objetivo) no instante $k$ \\
\hline$V_{2 a, k}$ & Custo total (função objetivo) do problema P2a no instante $k$ \\
\hline$V_{2 b, k}$ & Custo total (função objetivo) do problema $\mathrm{P} 2 \mathrm{~b}$ no instante $k$ \\
\hline$V(x, u)$ & Função de Lyapunov \\
\hline$x$ & Vetor de estados do sistema \\
\hline$X$ & Bloco com as componentes $x^{s}, x^{d}, x^{w}, x^{i}$ \\
\hline$x^{d}$ & Estado que calcula a evolução dos modos estáveis do sistema \\
\hline$x_{F}$ & Estado fictício utilizado no cálculo do filtro heurístico \\
\hline$x^{i}$ & Estado que calcula a evolução dos modos integradores do sistema \\
\hline$x_{a}^{i}\left(x_{b}^{i}\right)$ & Estado $x^{i}$ calculado no problema P2a (resp. P2b) \\
\hline
\end{tabular}




$\begin{array}{cl}x^{s} & \text { Predição da saída do sistema em estado estacionário } \\ y & \text { Vetor de saídas do sistema } \\ \bar{y} & \text { Vetor de predição das saídas do sistema } \\ y_{e} & \text { Vetor de saídas do sistema estendido, incorporando os estados } z \\ y(k+i \mid k) & \text { Previsão no instante } k \text { da saída no instante } k+i \\ y_{\min } & \text { Limite inferior da zona desejada para as saídas } \\ y_{\max } & \text { Limite superior da zona desejada para as saídas } \\ y^{s p} & \text { Set-point para a saída } \\ y_{s p, k} & \text { Set-point calculado pelo controlador no instante } k \\ Z & \text { Bloco com as componentes } z_{1}, \cdots, z_{\theta_{\max }} \text { e } z_{1}^{w}, \cdots, z_{\tau_{\max }}^{w} \\ z_{l} & \text { Estado que armazena a ação de controle ocorrida há } l \text { períodos de } \\ & \text { amostragem } \\ z_{l}^{w} & \text { Estado que armazena a variação nos distúrbios medidos ocorrida há } l \\ & \text { períodos de amostragem }\end{array}$

Símbolos utilizando alfabeto grego

$\begin{array}{cl}\alpha & \text { Parte real do resíduo de uma função de transferência com pólos } \\ \beta & \text { complexos conjugados } \\ & \text { Parte imaginária do resíduo de uma função de transferência com } \\ \delta & \text { pólos complexos conjugados } \\ \delta_{i, k} & \text { Variável de folga (slack) } \\ \delta_{u, k} & \text { Variável de folga para os modos integradores } \\ & \text { desejado } \\ \delta_{y, k} & \text { Variável de folga para o desvio entre o valor da saída e o set-point } \\ \Delta K_{i, j} & \text { calculado } \\ \Delta t & \text { Incerteza relativa ao valor de } K_{i, j} \\ \Delta u & \text { Tempo de amostragem } \\ \Delta u_{k} & \text { Variação na entrada (ação de controle) }\end{array}$




$\begin{array}{cl}\Delta u_{k}^{a} & \text { Vetor de ações de controle do problema P2a em todo o horizonte } m \\ \Delta u_{k}^{b} & \text { Vetor de ações de controle do problema P2b em todo o horizonte } m \\ \Delta u_{\max } & \text { Maior variação admissível nas ações de controle } \\ \Delta U_{\max } & \text { Vetor contendo a maior variação admissível nas ações de controle } \\ & \text { em todo o horizonte de controle } \\ \Delta w & \text { Variação nos distúrbios medidos } \\ \Delta \tau_{i, j, k} & \text { Incerteza relativa ao valor de } \tau_{i, j, k} \\ \theta_{i, j} & \text { Tempo morto da função de transferência } G_{i, j}(s) \\ \theta_{\max } & \text { Maior tempo morto entre as entradas manipuladas e saídas } \\ \lambda & \text { Autovalor a ser minimizado no cálculo do filtro heurístico } \\ \tau_{i, j} & \text { Tempo morto da função de transferência } G_{i, j}^{w}(s) \\ \tau_{i, j, k} & \text { k-ésima constante de tempo da expansão em frações parciais da } \\ \tau_{\max } & \text { função de transferência } G_{i, j}(s) \\ \Phi^{w} & \text { Maior tempo morto entre os distúrbios medidos e saídas } \\ \Phi^{w} & \text { Matriz auxiliar para construção de } \Psi \\ \Psi^{w} & \text { Matriz auxiliar para construção de } \Psi^{w} \\ & \text { Matriz que relaciona as saídas com a componente } x^{d} \\ & \text { transferência }\end{array}$

Sobrescritos

Transposta de uma matriz

Valor estimado

Complexo conjugado

Valores correspondentes a distúrbios medidos

Solução ótima

Solução viável 


\section{SUMÁRIO}

1. INTRODUÇÃO

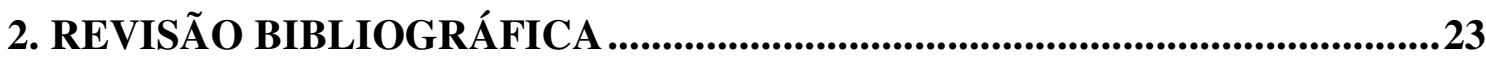

2.1. HISTÓRICO DE CONTROLADORES PREDITIVOS.......................................... 23

2.2. PROCESSOS INTEGRADORES..................................................................... 27

2.3. OBSERVADORES DE ESTADO.................................................................. 29

2.4. CONTROLE POR FAIXAS ........................................................................... 32

2.5. DESIGUALDADES MATRICIAIS.................................................................... 33

3. IHMPC PARA SISTEMAS INTEGRADORES E COM TEMPO MORTO ...36

3.1. MODELO EM ESPAÇO DE ESTADOS .................................................................... 36

3.1.1. Modelo Contendo Apenas Estados Reais ..................................................................................4

3.2. MODELO CONSIDERANDO DISTÚRBIOS MEDIDOS....................................... 45

3.3. DETECTABILIDADE DOS MODELOS PROPOSTOS ........................................50

3.4. FORMULAÇÃO DO PROBLEMA EM 1 PASSO ...................................................53

3.5. FORMULAÇÃO DO PROBLEMA EM 2 PASSOS.................................................5 55

3.6. REPRESENTAÇÃO DOS PROBLEMAS DE OTIMIZAÇÃO COMO

PROGRAMAÇAO QUADRÁTICA ………………………………………………..... 57

3.6.1. Transformando P2a em um problema de programação quadrática ............................................ 57

3.6.2. Transformando P2b num problema de programação quadrática .................................................60

3.6.3. Transformando P1 em um problema de programação quadrática ................................................. 68

4. DESENVOLVIMENTO DE UM OBSERVADOR HEURÍSTICO ...................70

4.1. FILTRO DE KALMAN ……………………………........................................ 70

4.2. OBSERVADOR HEURÍSTICO .................................................................... 71

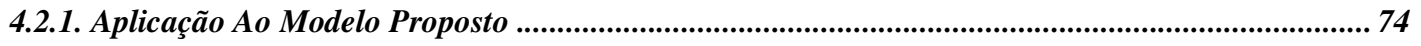

5. ANÁLISE DE ESTABILIDADE DO CONTROLADOR PROPOSTO.............77 
6. SIMULAÇÕES E ANÁLISES NUMÉRICAS......................................................83

6.1. ESTUDO DE CASO BASEADO NUM SISTEMA REAL ...................................... 83

6.2. COMPARAÇÃO ENTRE AS FORMULAÇÕES COM 1 E 2 PASSOS ..................... 85

6.3. COMPARAÇÃO ENTRE OBSERVADOR HEURÍSTICO E FILTRO DE KALMAN

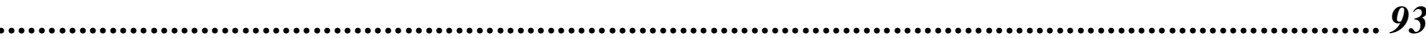

6.3.1. Caso 1: comparação entre o Filtro de Kalman e estado completamente medido..............................93

6.3.2. Caso 2: comparação entre o Filtro de Kalman e o observador heurístico com modelo nominal... 97

6.3.3. Caso 3: comparação entre o Filtro de Kalman e o observador heurístico com modelo incorreto 102

7. CONSIDERAÇÕES FINAIS ...........................................................................107

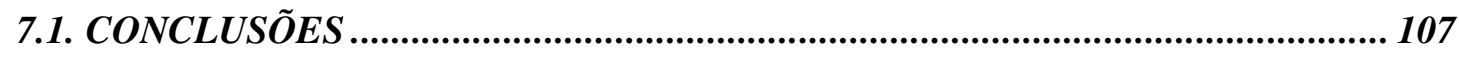

7.2. SUGESTÕES DE CONTINUIDADE ………………………………………..... 108

REFERÊNCIAS ..................................................................................................................110 


\section{INTRODUÇÃO}

O controle de processos na indústria química é uma ferramenta fundamental para questões de segurança, respeito à legislação ambiental e operação lucrativa de uma planta. No caso de plantas complexas, com muitas variáveis que interagem entre si, as estratégias de controle regulatório clássico não são suficientes para obter a máxima lucratividade possível. A partir do final dos anos 1970, com as crises do petróleo, buscaram-se soluções para o controle de refinarias de modo a aumentar a eficiência dos processos através da operação em condições mais próximas dos limites dos equipamentos. Nesse contexto foi desenvolvido o Controle por Matriz Dinâmica (Dynamic Matrix Control ou DMC na sigla em inglês), dando origem ao controle preditivo baseado em modelos (Model Predictive Control ou MPC).

Habitualmente utiliza-se uma estrutura de controle dividida em camadas. Em um primeiro nível de planejamento são calculadas as condições operacionais que conduzirão à produção mais lucrativa possível. Essas condições são transmitidas à camada de controle preditivo (supervisório), a qual calcula as melhores ações de controle a tomar em cada instante de amostragem. Por fim, essas ações levam à mudança de valores objetivo (set-points) da camada de controle regulatório, que mantém, de fato, a operação da planta o mais próximo possível das condições ótimas.

O fundamento do controle preditivo é a utilização de um modelo da planta para estimar o estado futuro para uma determinada alteração nas variáveis manipuladas do processo. Definese uma função objetivo para mensurar o resultado da aplicação de uma mudança nas entradas do processo. Em termos simples, essa função objetivo é composta por duas parcelas: uma medida da distância entre o estado previsto e o estado desejado da planta e outra considerando o esforço de controle necessário.

Formulando-se a situação descrita anteriormente em termos rigorosos, obtém-se um problema de otimização em que são calculadas as entradas futuras da planta de modo a minimizar essa

função custo, respeitando-se as restrições do processo como saturação dos atuadores e limites operacionais dos equipamentos, entre outros.

Apesar da solução do problema ser um conjunto de entradas para os instantes futuros, apenas a entrada relativa ao instante atual é de fato aplicada na planta. No próximo instante de amostragem repete-se todo o procedimento de cálculo e de implementação apenas da entrada relativa àquele instante. Essas predições são realizadas em malha aberta, no sentido que os erros que ocorrerão entre as saídas futuras e seus valores previstos não são utilizados no 
cálculo. Assim, as predições poderiam se distanciar da realidade para horizontes de predição muito grandes, daí a vantagem de implementar apenas a entrada sobre a qual há menos incerteza.

Essa maneira de recalcular as ações de controle caracteriza um dos princípios mais importantes dos controladores preditivos, que é o horizonte rolante (rolling horizon ou moving horizon). Com essa retroalimentação, é possível lidar com incertezas no modelo, inerentes a qualquer representação da planta.

Algumas das principais vantagens da utilização de um controle preditivo baseado em processo são: consideração explícita das restrições do processo, evitando que a planta seja levada a operar em condições fora das faixas de segurança ou de limites ambientais; facilidade em lidar com o caso de sistema com várias entradas e saídas (Multiple Input, Multple Output $M I M O)$, que é tratado por procedimento virtualmente idêntico ao de sistemas com uma entrada e uma saída (Single Input, Single Output - SISO); tratamento de tempos mortos de forma natural, já que eles podem ser incorporados ao modelo do sistema.

Por outro lado, ainda há algumas questões que limitam o uso da técnica. Em primeiro lugar, o sucesso de uma estratégia de controle preditivo depende da existência de um modelo preciso e atualizado, cuja obtenção e manutenção podem ser demasiadamente custosas. Além disso, como é necessário resolver em tempo real um problema de otimização com algumas centenas ou milhares de variáveis, restringe-se a aplicação a processos de dinâmica lenta, o que não é grande inconveniente para a indústria química.

O presente trabalho apresenta um controlador preditivo para sistemas integradores e com tempo morto. Uma das principais propriedades deste controlador é a garantia de estabilidade quando o processo representado pelo modelo for nominal, ou seja, o modelo reproduzir exatamente o comportamento da planta. O controlador exige o uso de um estimador de estados como um Filtro de Kalman e, a partir de medidas dos valores de saída, é então possível estimar os estados internos da planta, possibilitando a implantação deste controlador em sistemas industriais em que não se tem acesso direto aos estados. Este documento dividese em 7 seções: na seção 2 é feita uma revisão da literatura de controle preditivo, apresentando as principais formulações e resultados teóricos obtidos nas últimas décadas. É abordado também o tópico de observadores de estado e são fornecidos fundamentos teóricos da teoria de desigualdades matriciais.

Em seguida, a seção 3 apresenta com detalhes um modelo usado para representar sistemas com tempo morto e/ou integradores. São descritas duas variações desse modelo: a primeira 
delas permite a incorporação de distúrbios medidos e a segunda é um rearranjo de matrizes para evitar a presença de números imaginários no modelo. Ainda na seção 3 é formulado um controlador preditivo nominalmente estável, baseado nos modelos desenvolvidos anteriormente. A seção 4 apresenta duas estratégias distintas que podem ser empregadas no problema de síntese de um observador de estados para o sistema. A primeira estratégia é baseada no Filtro de Kalman e a segunda é uma proposição de um observador heurístico. Na seção 5 é apresentada a dedução formal da estabilidade do controlador quando o modelo empregado é exato. Já na seção 6 são mostrados resultados de simulações feitas com este controlador. Comparam-se em primeiro lugar as duas formulações do controlador apresentadas na seção 3 e também os dois observadores definidos na seção 4. Por fim, a seção 7 contém as conclusões deste trabalho e uma indicação de possíveis continuações da linha de pesquisa. 


\section{REVISÃO BIBLIOGRÁFICA}

\subsection{HISTÓRICO DE CONTROLADORES PREDITIVOS}

Historicamente, os primeiros desenvolvimentos na área de controle preditivo baseado em modelos ocorreram a partir do final dos anos 1970, com a aparição do Model Predictive Heuristic Control (Controle heurístico com predição baseada em modelo) em Richalet et al. (1978). Alguns dos principais pontos das estratégias de controle preditivo aparecem claramente já neste trabalho pioneiro: facilidade em abordar problemas com diversas entradas e saídas, o uso de um modelo interno para prever as relações entre entradas e saídas e a especificação de uma trajetória de referência que se tenta alcançar.

O modelo interno utilizado é baseado na resposta impulsiva do sistema, demandando o armazenamento de uma grande quantidade de valores de modo a reproduzir a dinâmica do sistema em todo o intervalo entre uma perturbação e a estabilização posterior. Como o próprio nome indica, esta estratégia emprega métodos heurísticos para calcular as melhores entradas, sem formular formalmente um problema de otimização. A estratégia de horizonte móvel, em que a cada tempo de amostragem são recalculadas as entradas ótimas com base na atualização das medidas da planta, também está presente.

Posteriormente, com o desenvolvimento do DMC em Cutler e Ramaker (1980), passa-se a calcular as melhores entradas a partir de um problema de otimização com restrições com função objetivo equivalente a:

$$
\min _{\Delta u} V_{k}=\sum_{i=0}^{H p}\left\|y^{s p}-y(k+i \mid k)\right\|_{Q}^{2}+\sum_{j=0}^{H c}\|\Delta u(k+j \mid k)\|_{S}^{2}
$$

Sendo:

\begin{tabular}{|l|l|}
\hline$k$ & Instante atual \\
\hline$H p$ & Horizonte de predição \\
\hline$H c$ & Horizonte de controle \\
\hline$y(k+i \mid k)$ & Previsão no instante $k$ da saída no instante $k+i$ \\
\hline$y^{s p}$ & Set-point para a saída \\
\hline
\end{tabular}




\begin{tabular}{|l|l|}
\hline$\Delta u$ & Variação na entrada (ação de controle) \\
\hline$Q, S$ & Pesos da função objetivo \\
\hline$V_{k}$ & Custo total no instante $k$ \\
\hline
\end{tabular}

Colocando-se restrições lineares sobre os valores das entradas, das saídas e dos esforços de controle, além das relações lineares entre entradas e saídas, tem-se um problema de programação quadrática (Quadratic programming, QP na sigla em inglês).

Uma alternativa em relação ao modelo baseado na resposta impulsiva é o uso de uma realização em espaço de estados. De acordo com Morari e Lee (1999), essa abordagem em estados tornou-se dominante na literatura de controle preditivo. Representa-se um sistema linear variante no tempo discreto na seguinte forma:

$$
\begin{aligned}
& x(k+1)=A(k) x(k)+B(k) u(k) \\
& y(k)=C(k) x(k)
\end{aligned}
$$

Sendo:

\begin{tabular}{|l|l|}
\hline$k$ & Instante atual \\
\hline$x$ & Vetor de estados do sistema $\left(x \in \mathbb{R}^{n}\right)$ \\
\hline$u$ & Vetor de entradas do sistema $\left(u \in \mathbb{R}^{n u}\right)$ \\
\hline$y$ & Vetor de saídas do sistema $\left(y \in \mathbb{R}^{n y}\right)$ \\
\hline$A, B, C$ & Matrizes de dimensões apropriadas que caracterizam a dinâmica do sistema \\
\hline
\end{tabular}

No caso particular de sistemas invariantes no tempo, as matrizes $A, B, C$ não dependem do instante de amostragem $k$. Neste trabalho serão abordados somente sistemas invariantes no tempo.

Uma das principais vantagens da substituição de modelos baseados na resposta impulsiva ou resposta ao degrau por aqueles em variáveis de estado é a economia de informação que deve ser mantida em memória para o cálculo das predições. Outro benefício é a possibilidade de utilizar conhecimentos clássicos da teoria de sistemas lineares de modo direto para analisar um sistema.

Devido à presença de restrições que podem estar ou não ativas em um dado instante, não é possível determinar uma expressão analítica para a lei de controle. Com isso, o estudo da 
estabilidade de controladores preditivos baseados em modelo só foi possível com a utilização de métodos indiretos como os baseados em funções de Lyapunov. Conforme definido em Maciejowski (2002), uma função $V(x, u)$ positiva definida é dita função de Lyapunov quando as seguintes condições são satisfeitas:

a) Se $\left\|x_{1}^{T}, u_{1}^{T}\right\|>\left\|x_{2}^{T}, u_{2}^{T}\right\|$ então $V\left(x_{1}, u_{1}\right) \geq V\left(x_{2}, u_{2}\right)$;

b) Ao longo de qualquer trajetória do sistema $x(k+1)=f(x(k), u(k))$ numa vizinhança da origem, $V(x(t+1), u(t+1)) \leq V(x(t), u(t))$.

Keerthi e Gilbert (1988) demonstraram a estabilidade de um controlador preditivo desde que se inclua uma restrição sobre o valor terminal do estado. Impondo-se que o estado está na origem ao final do horizonte preditivo, a prova de estabilidade decorre naturalmente. No entanto, essa abordagem possui uma limitação importante em termos de factibilidade do problema de otimização. Como normalmente as entradas estão em faixas limitadas de atuação, é possível mostrar que existe um estado a partir do qual a origem não pode ser alcançada dentro do horizonte de predição, o qual é finito. Esse algoritmo não é diretamente aplicado a um controlador industrial pois um distúrbio poderia levar a planta a regiões onde o problema de otimização é infactível.

O argumento principal para a demonstração de estabilidade feita por Keerthi e Gilbert (1988) é a identificação da própria função objetivo como uma função de Lyapunov, o que garante a estabilidade do controlador.

Uma extensão desta técnica foi apresentada em Michalska e Mayne (1993) modificando a restrição sobre a condição do estado ao final do horizonte preditivo ao invés de determinar que o estado deverá se encontrar exatamente sobre a origem, impõe-se que ele deve estar no interior de um conjunto $\mathrm{S}$ que engloba a origem. Este conjunto deve ser invariante, no sentido de que uma vez que o estado esteja em seu interior sua evolução não forçada para qualquer instante posterior permanecerá nessa região.

Uma vez que o estado encontre-se no interior desse conjunto, é aplicada uma realimentação que o conduzirá à origem. Esse tipo de controlador é denominado MPC dual por ter duas regras de controle diferentes, dentro e fora do conjunto $S$.

No entanto, mesmo com o aumento significativo da região que o estado deve alcançar ao término do horizonte preditivo em relação à proposição de Keerthi e Gilbert (1988), distúrbios 
suficientemente grandes ainda podem deixar o estado em um ponto a partir do qual a região terminal não é alcançável dentro do horizonte necessário, por causa das limitações na entrada da planta. Portanto, a estratégia para garantir estabilidade diante de qualquer magnitude de distúrbio precisa ser modificada.

Rawlings e Muske (1993) mostraram que uma demonstração de estabilidade pode ser obtida ao se considerar um controlador preditivo baseado em modelo com horizonte de predição infinito (IHMPC, na sigla em inglês). Primeiramente, mostra-se que caso o sistema esteja em uma condição tal que o problema de otimização é viável no instante $k$, então o problema no instante $k+1$ também será viável, o que é chamado de factibilidade recursiva. Além disso, pode-se ver que nessas condições o valor da função objetivo será estritamente decrescente caso o sistema não tenha atingido o estado estacionário. A estabilidade assintótica do controlador decorre da observação de que a função objetivo é uma função de Lyapunov do sistema.

Quando se usa um horizonte infinito de predição, a função objetivo passa a conter uma série infinita de termos relacionados ao erro entre saídas previstas e o sinal de referência. Para tornar o problema numericamente tratável, essa série é dividida em duas contribuições: a primeira é o somatório dos erros durante o horizonte de controle e a segunda é um custo terminal. Este custo terminal pode ser calculado a partir de uma matriz de pesos apropriadamente definida e do estado previsto ao final do horizonte de controle. Com essa manipulação, o problema torna-se simplesmente uma questão de programação quadrática cuja abordagem computacional está bem estabelecida.

Esta abordagem estava inicialmente limitada ao problema de rejeição de perturbações, não de acompanhamento da trajetória de referência (set-point tracking). Além disso, como o horizonte de controle utilizado permanecia finito, a técnica proposta só era válida para sistemas estáveis em malha aberta. O modelo utilizado em Rawlings e Muske (1993) para representar em tempo discreto a planta, suposta linear e invariante no tempo, era da forma tradicional em espaço de estados (posicional). Este tipo de modelo é bastante adequado para o caso regulador (rejeição de perturbações), em que o estado estacionário é conhecido previamente e por isso pode ser feita uma mudança de coordenadas de modo a deixá-lo na origem do novo sistema de coordenadas.

$\mathrm{Na}$ indústria de processos químicos é comum a mudança dos set-points das saídas, sendo esses valores normalmente calculados numa camada de otimização econômica da planta. Como os modelos identificados sempre apresentam algum grau de incerteza, não é possível 
determinar exatamente, a partir da equação do modelo, o valor necessário de entrada para gerar uma determinada saída.

Numa tentativa de contornar essa dificuldade, Lee, Morari e Garcia (1994) propuseram uma nova formulação para o sistema denominada incremental, usando variações nas entradas e não as entradas propriamente ditas:

$$
\begin{aligned}
& x(k+1)=A x(k)+B \Delta u(k) \\
& y(k)=C x(k)
\end{aligned}
$$

Qualquer uma das formas pode ser facilmente convertida na outra. Portanto a escolha entre (2-2) ou (2-3) é feita apenas baseando-se apenas no objetivo que se deseja alcançar, não havendo nenhuma dificuldade técnica adicional quando utilizada a forma (2-3).

Com essa formulação incremental qualquer estado estacionário corresponde a $\Delta u=0$ e a algum valor de $u$ que não precisa ser explicitamente calculado pelo controlador. Isso permitiria, em princípio, criar um controlador preditivo capaz de acompanhar mudanças no valor de referência das saídas. No entanto, ao utilizar esse modelo incremental num controlador preditivo, são acrescentados modos integradores mesmo quando a planta é originalmente estável em malha aberta. Em decorrência disso, a garantia de estabilidade do controlador não é uma questão trivial a princípio, apesar do uso de um horizonte infinito. Torna-se necessário utilizar alguma estratégia similar àquela de Muske e Rawlings (1993), zerando os modos integradores ao final do horizonte de controle.

O intuito desta seção é dar uma visão geral do uso e evolução dos controladores preditivos nas últimas décadas. Uma discussão mais ampla do assunto pode ser encontrada em Garcia, Prett e Morari (1989), Morari e Lee (1999) ou Camacho e Bordons (2004).

\subsection{PROCESSOS INTEGRADORES}

$\mathrm{Na}$ indústria química são freqüentes os processos integradores, seja devido à presença de tanques e vasos ou a correntes de reciclo. Como as primeiras demonstrações de estabilidade de controladores preditivos repousavam sobre a hipótese de estabilidade dos sistemas em malha aberta, foi preciso criar uma estratégia diferente para esses casos. Rodrigues e Odloak (2003) propuseram a divisão da otimização em dois subproblemas: o primeiro tenta zerar os modos integradores e o segundo é um MPC tradicional para levar as saídas aos valores 
desejados. A idéia de dividir o problema do MPC em dois passos com objetivos distintos havia primeiramente sido introduzida, em outro contexto, por Lee e Xiao (2000).

No contexto das primeiras formulações de controle preditivo, como no caso do DMC, sistemas integradores eram tratados apenas de forma aproximada. O modelo usado nessas formulações era a convolução das entradas previstas com coeficientes obtidos a partir da resposta a uma perturbação do tipo degrau. Entretanto, como a saída de um sistema integrador diverge quando excitado por esse tipo de perturbação, os coeficientes da resposta ao degrau eram truncados após um número suficientemente grande de períodos de amostragem. Obviamente, não há garantia de estabilidade para controladores formulados dessa maneira. Formulações mais rigorosas começaram a ser desenvolvidas em Muske e Rawlings (1993). Neste trabalho aponta-se que os modos instáveis devem ser zerados após o horizonte de controle, pois, caso contrário, eles continuarão evoluindo em função do tempo e não convergirão para 0 . No entanto, essa restrição provoca uma grande redução da região viável do problema de otimização, o que pode inviabilizar o uso do controlador. Por outro lado, é apresentada uma demonstração da factibilidade recursiva do controlador proposto quando o modelo do controlador é exato (caso nominal).

Rodrigues e Odloak (2003) desenvolveram uma nova realização em espaço de estados para sistemas integradores que permite provar a estabilidade nominal para um controlador preditivo baseado em um modelo com essa realização. Essa realização é lida com o tempo de forma contínua, o que não é o mais freqüente na literatura de controle preditivo. São impostas restrições terminais sobre os modos integradores, tanto os naturais do sistema quanto aqueles introduzidos pela representação em velocidade.

Para aumentar a região viável, é colocada uma variável de folga ou slack ( $\delta$ ) na restrição que impõe o valor terminal para as saídas. Esse tipo de procedimento é bastante comum, garantindo que a restrição seja sempre respeitada. Por outro lado, penaliza-se o valor da variável de folga na função objetivo, de modo que a solução tenha valores diferentes de zero para essa variável apenas se não houver uma solução viável com $\delta=0$.

Carrapiço e Odloak (2005) estenderam o procedimento de Rodrigues e Odloak (2003) para desenvolver um controlador preditivo nominalmente estável para sistemas integradores. Mostrou-se que é possível incluir variáveis de folga para relaxar a restrição terminal sobre os modos integradores e ainda assim manter a estabilidade nominal do controlador. Isso não é uma extensão trivial do trabalho de Rawlings e Muske (1993) porque quando as variáveis de decisão incluem slacks dos modos integradores, a solução do instante $k$ não permite a 
determinação de um majorante para a função objetivo no instante $k+1$. Portanto, não se consegue encontrar facilmente uma função de Lyapunov para o sistema e a questão de estabilidade permanece não resolvida.

Em Carrapiço e Odloak (2005) foram utilizados dois procedimentos para contornar essa dificuldade. No primeiro deles, foi adicionada uma restrição de contração do valor da norma das variáveis de folga relacionadas aos integradores. Assim, impõe-se de forma indireta que essas variáveis devem tender a 0 e, conseqüentemente, os modos integradores também tendem a 0 .

$\mathrm{Na}$ outra abordagem, o problema de otimização foi dividido em dois subproblemas: no primeiro, foram calculadas ações de controle de modo a minimizar as variáveis de folga dos modos integradores. O segundo problema é similar a um MPC padrão, diferindo apenas pela inclusão de uma restrição que força as contribuições sobre os modos integradores das ações de controle a serem iguais às contribuições obtidas no primeiro problema.

Os dois problemas são sempre viáveis e o resultado é que, caso não haja distúrbios, após um tempo finito é atingido um estado a partir do qual os modos integradores podem ser zerados dentro de um horizonte de controle. Assim, concilia-se a formulação tradicional de MPC para sistemas instáveis com a garantia de viabilidade do problema resolvido pelo otimizador.

González, Marchetti e Odloak (2007) estenderam de certa maneira o trabalho de Carrapiço e Odloak (2005) de modo a garantir a robustez do controlador. O modelo utilizado para o sistema parte do pressuposto que o modelo da planta real é desconhecido mas está localizado no interior de um politopo cujos vértices são modelos conhecidos.

González, Marchetti e Odloak (2007) também usaram um controlador com dois passos, o primeiro responsável por zerar os modos integradores e o segundo busca conduzir o sistema ao ponto operacional desejado. A estabilidade robusta é garantida ao impor a contração da função objetivo em todo o conjunto de modelos conhecidos. Dessa forma, a função objetivo que seria calculada se o modelo da planta real fosse conhecido também é decrescente. No entanto, a formulação do controlador é feita apenas para sistemas sem tempos mortos .

\subsection{OBSERVADORES DE ESTADO}

Quando se utiliza um modelo em espaço de estados para representar um sistema real, este modelo é uma aproximação da realidade. Mesmo assumindo que o sistema se comporte de modo linear em toda a região de interesse, o que é uma hipótese bastante restritiva, seria 
necessário conhecer com exatidão todos os parâmetros das matrizes $A, B, C$ para obter um modelo capaz de reproduzir perfeitamente a dinâmica do sistema. Finalmente, mesmo se esses parâmetros fossem determinados exatamente, o comportamento mensurável da planta poderia ser diferente do calculado pelo modelo devido a erros aleatórios inerentes ao processo de medição.

Em resumo, por causa de simplificações ou erros de modelagem e de imprecisões nas medidas, o modelo não prevê completamente a evolução do sistema real. Fica claro, portanto, que é necessário corrigir de alguma forma os estados calculados pelo modelo de modo a alcançar resultados mais próximos da planta real. Este problema levou ao desenvolvimento da teoria de estimação de estados, inicialmente pesquisada com o intuito de auxiliar os projetos aeroespaciais nas décadas de 1950 e 1960. Um dos marcos fundadores da teoria recente foi o desenvolvimento do filtro de Kalman, atualmente empregado em todos os campos de engenharia, economia, biologia e outras ciências. (Simon, 2006)

Para exemplificar a ação de um observador de estados, considere uma planta linear invariante no tempo cuja evolução é dada por:

$$
\begin{aligned}
& x(k+1)=A x(k)+B u(k) \\
& y(k)=C x(k)
\end{aligned}
$$

Admita que as matrizes $A, B, C$ são conhecidas e que se dispõe de medidas de $y$ e $u$ até o instante $k$. Caso se queira simular esse comportamento, é necessário também conhecer o estado $x$. Utiliza-se o símbolo ${ }^{\wedge}$ para indicar valores estimados, diferenciando-os dos valores reais. Suponha que apenas uma estimativa $\hat{x}$ esteja disponível, então a simulação daria resultados conforme as equações

$$
\begin{aligned}
& \hat{x}(k+1)=A \hat{x}(k)+B u(k) \\
& \hat{y}(k)=C \hat{x}(k)
\end{aligned}
$$

Uma medida do erro da estimativa é a comparação entre a saída real e a saída simulada, $y(k)-C \hat{x}(k)$. Esse erro pode ser utilizado como retroalimentação para corrigir (2-5):

$$
\hat{x}(k+1)=A \hat{x}(k)+B u(k)+L(y(k)-C \hat{x}(k))
$$


Como definido em Glad e Ljung (1999), a matriz $L \in \mathbb{R}^{n \times n y}$ é o ganho do observador. Seja $e(k)=x(k)-\hat{x}(k)$ o erro entre a estimativa e o estado real. Utilizando essa definição em (2-6) e simplificando a expressão obtida, tem-se

$$
\begin{aligned}
& x(k+1)-e(k+1)=A(x(k)-e(k))+B u(k)+L(y(k)-C(x(k)-e(k))) \\
& e(k+1)=(A-L C) e(k)
\end{aligned}
$$

Logo, quando os autovalores de $(A-L C)$ estão localizados estritamente no interior do círculo unitário o erro tende a zero. Em outros termos, a estimativa do estado tende assintoticamente ao valor real. Pode-se demonstrar que existe ao menos uma matriz $L$ satisfazendo à condição dos autovalores se e somente se o sistema for detectável (Hespanha, 2009). A escolha da matriz $L$ deve levar em conta dois fatores conflitantes: por um lado, quanto mais próximos os autovalores estiverem da origem, mais rápida a convergência do erro para zero. Contudo, esses autovalores muito pequenos levam a estimativa a oscilar bastante na presença de ruídos. Uma solução ótima, num certo sentido, para esse conflito é o Filtro de Kalman. Ele pressupõe que se tenha um modelo dos ruídos, permitindo quantificar a sensibilidade do observador a essas variações.

A descrição do Filtro de Kalman apresentada a seguir foi adaptada de Glad e Ljung (1999). Considera-se inicialmente um sistema linear, invariante no tempo, em tempo discreto da forma

$$
\begin{aligned}
& x(k+1)=A x(k)+B u(k)+N v_{1}(k) \\
& y(k)=C x(k)+v_{2}(k)
\end{aligned}
$$

em que $v_{1}(k)$ e $v_{2}(k)$ são processos estocásticos, denominados, respectivamente, ruído do processo e ruído de medida. Esses processos são considerados ruídos brancos, gaussianos, de média nula e não auto-correlacionados. Sejam $R_{1}, R_{2}$ suas matrizes de covariância e $R_{12}$ a matriz de correlação cruzada.

O ganho do filtro é calculado de modo que a esperança do erro de estimativa seja mínimo, ou seja, 


$$
\min E\left[(x(k)-\hat{x}(k)) \cdot(x(k)-\hat{x}(k))^{T}\right]
$$

Pode-se demonstrar que para $k$ suficientemente grande este ganho é dado por:

$$
K_{K}=\left(A P C^{T}+N R_{12}\right)\left(C P C^{T}+R_{2}\right)^{-1}
$$

em que $P$, a matriz de covariância do erro de estimação, é a solução da equação

$$
P=A P A^{T}+N R_{1} N^{T}-\left(A P C^{T}+N R_{12}\right)\left(C P C^{T}+R_{2}\right)^{-1}\left(A P C^{T}+N R_{12}\right)^{T}
$$

\subsection{CONTROLE POR FAIXAS}

Apesar das primeiras formulações de controladores preditivos baseados em modelos admitirem que o objetivo do controlador é manter as variáveis controladas iguais a valores de referência pré-determinados (Maciejowski, 2002), há exemplos de sistemas reais em que essa premissa não é válida. Por exemplo, no caso do controle de nível de um tanque, é fundamental que a variável controlada, o nível permaneça dentro de limites mínimo e máximo estabelecidos anteriormente. Contudo, pode não haver necessidade de estabelecer um valor ótimo para o nível do tanque, já que qualquer nível dentro dos limites já é adequado para o bom funcionamento da planta.

Outros casos de variáveis em que o controle por faixas é uma estratégia adequada foram abordados em Zanin, Tvrzska de Gouvêa e Odloak (2002), como por exemplo a temperatura no reator ou nos diferentes estágios do regenerador de uma unidade de craqueamento catalítico em leito fluidizado (FCC).

Numa estratégia de controle em camadas, há uma camada de otimização que, a partir do planejamento de longo prazo da planta, calcula o ponto de operação mais adequado do ponto de vista econômico. Essa camada de otimização fornece, portanto, valores ótimos para as entradas e saídas das diversas unidades da planta, que podem ser utilizados na camada de controle avançado. Esse esquema está sintetizado na Figura 2-1: 


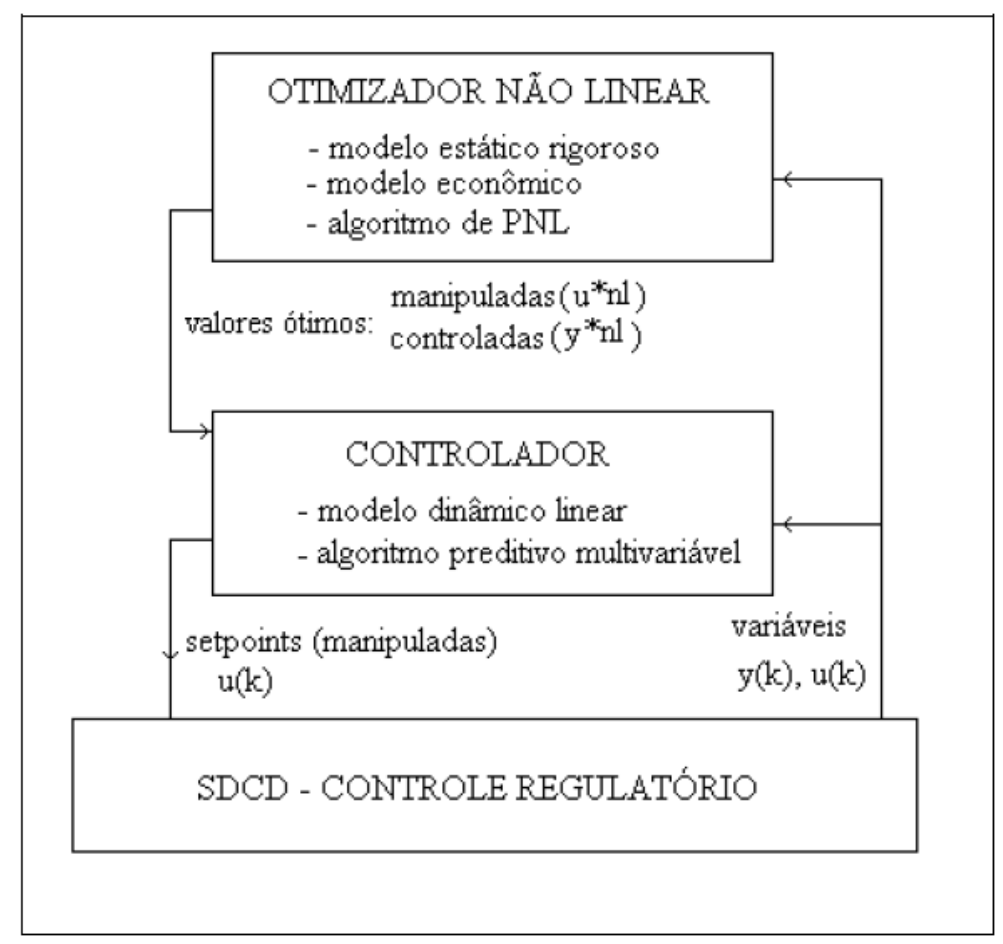

Figura 2-1: Estrutura de controle em camadas, Zanin (2001)

Na formulação proposta em González e Odloak (2009) a função objetivo do controlador penaliza desvios das entradas do sistema em relação aos valores desejáveis calculados na camada de otimização (targets). Por outro lado, as saídas do sistema são penalizadas na função objetivo apenas se estiverem fora de uma faixa de operação que engloba o valor ótimo para cada saída. Essa abordagem fornece mais graus de liberdade ao controlador do que a maneira tradicional de impor uma trajetória de referência.

\subsection{DESIGUALDADES MATRICIAIS}

De acordo com Boyd et al. (1994), uma desigualdade matricial linear (Linear Matrix Inequality, LMI na sigla em inglês) pode ser definida como:

$$
F(x)=F_{0}+\sum_{i=1}^{m} x_{i} F_{i}>0
$$


em que $x \in \mathbb{R}^{m}$ é uma variável e as matrizes simétricas $F_{i} \in \mathbb{R}^{n \times n}, i=0, \cdots, m$ são constantes. O símbolo ">" é utilizado, por abuso de linguagem, para representar que a matriz $F(x)$ é positiva definida.

Apesar da forma aparentemente limitada dessa definição de LMI, uma restrição na forma (2-12) pode representar diversas situações comuns em problemas de controle. Conforme exemplificado em Boyd et al. (1994), essa restrição pode ser modificada de maneira que a variável do problema seja uma matriz, e não um simples vetor. Pode-se mostrar que o conjunto de pontos $x \in \mathbb{R}^{m}$ que satisfaz (2-3) é convexo.

Um dos resultados fundamentais da teoria de LMI é o Complemento de Schur, que estabelece a equivalência entre uma desigualdade não linear e uma LMI. O enunciado preciso desse lema é dado a seguir:

Complemento de Schur: sejam $Q(x)=Q(x)^{T}, R(x)=R(x)^{T}, S(x)$ matrizes que dependem de $\mathrm{x}$ de maneira afim. Então as desigualdades

$$
R(x)>0, Q(x)-S(x) R(x)^{-1} S(x)^{T}>0
$$

são equivalentes à LMI

$$
\left[\begin{array}{ll}
Q(x) & S(x) \\
S(x) & R(x)
\end{array}\right]>0
$$

Uma demonstração pode ser vista em VanAntwerp e Braatz (2000).

Esse resultado é particularmente útil para a análise da estabilidade de um sistema. Um sistema linear na forma (2-3) é estável em malha aberta se e somente se existe uma matriz $P$ simétrica positiva definida tal que a seguinte Equação de Lyapunov tem solução (Hespanha, 2009):

$$
A^{T} P A-P<0
$$

Através do Complemento de Schur, a equação na forma (2-15) pode ser transformada numa LMI. A principal vantagem dessa transformação é a existência de algoritmos eficientes para a 
solução numérica de problemas de otimização com função objetivo linear e restrições que são LMIs. Uma discussão mais completa desses algoritmos foi feita em Boyd et al. (1994).

Outra classe de desigualdades matriciais é a desigualdade matricial bilinear (Bilinear Matrix Inequality, BMI na sigla em inglês). Conforme VanAntwerp e Braatz (2000), uma BMI pode ser escrita na forma:

$$
F(x, y)=F_{0}+\sum_{i=1}^{m} x_{i} F_{i}+\sum_{j=1}^{n} y_{j} G_{j}+\sum_{i=1}^{m} \sum_{j=1}^{n} x_{i} y_{j} H_{i j}>0
$$

As variáveis de uma BMI são os vetores $x \in \mathbb{R}^{m}$ e $y \in \mathbb{R}^{m}$, enquanto as matrizes simétricas $F_{i} \in \mathbb{R}^{n \times n}, G_{j} \in \mathbb{R}^{n \times n}, H_{i j} \in \mathbb{R}^{n \times n}(i=0, \cdots, m ; j=1, \cdots, n)$ são constantes.

Ao contrário das LMIs, o conjunto de pontos $x \in \mathbb{R}^{m}, y \in \mathbb{R}^{m}$ que satisfaz (2-16) não é convexo. Em decorrência desse fato, os algoritmos existentes para resolução de BMIs são menos eficientes que aqueles voltados para LMIs. 


\section{IHMPC PARA SISTEMAS INTEGRADORES E COM TEMPO MORTO}

\subsection{MODELO EM ESPAÇO DE ESTADOS}

Considere um sistema com $n u$ entradas e $n y$ saídas, sendo $n i$ integradoras. Esse sistema pode ser representado por um modelo de funções de transferência $G(s)$ :

$$
G(s)=\left[\begin{array}{ccc}
G_{1,1}(s) & \cdots & G_{1, n u}(s) \\
\vdots & \ddots & \vdots \\
G_{n y, 1}(s) & \cdots & G_{n y, n u}(s)
\end{array}\right]
$$

Seja $n a$ a ordem das funções de transferência $G_{i, j}(s)$. A expressão dessas funções pode ser dada por:

$$
G_{i, j}(s)=\frac{b_{i, j, 0}+b_{i, j, 1} s+\cdots+b_{i, j, n b} s^{n b}}{s\left(s-r_{i, j, 1}\right)\left(s-r_{i, j, 2}\right) \cdots\left(s-r_{i, j, n a}\right)} e^{-\theta_{i, j} s}
$$

As respostas ao degrau unitário dessas funções de transferência podem ser obtidas a partir das expansões em frações parciais:

$$
S_{i, j}(s)=\frac{G_{i, j}(s)}{s}=\frac{d_{i, j}^{0}}{s} e^{-\theta_{i, j} s}+\frac{d_{i, j, 1}^{d}}{s-r_{i, j, 1}} e^{-\theta_{i, j} s}+\cdots+\frac{d_{i, j, n a}^{d}}{s-r_{i, j, n a}} e^{-\theta_{i, j} s}+\frac{d_{i, j}^{i}}{s^{2}} e^{-\theta_{i, j} s}
$$

Seja $\Delta t$ o período de amostragem. Assim, (3-3) pode ser reescrita em tempo discreto na seguinte forma:

$S_{i, j}(k \Delta t)=\left(d_{i, j}^{0}+d_{i, j, 1}^{d} e^{r_{i, j, 1} k \Delta t-\theta_{i, j}}+\cdots+d_{i, j, n a}^{d} e^{r_{i, j, n a} k \Delta t-\theta_{i, j}}+d_{i, j}^{i}\left(k \Delta t-\theta_{i, j}\right)\right) U\left(\Delta t-\theta_{i, j}\right)$,

sendo $U(t)$ a função-degrau, ou seja, 


$$
U(t)=\left\{\begin{array}{l}
0, \text { se } t<0 \\
1, \text { se } t \geq 0
\end{array},\right.
$$

Este sistema pode ser representado em espaço de estados com entradas incrementais:

$$
\begin{aligned}
& x(k+1)=A \quad x(k)+B \quad \Delta u(k) \\
& y_{e}(k)=C \quad x(k)
\end{aligned}
$$

Em que o estado $x$ é definido como

$$
\begin{gathered}
x(k)=\left[\begin{array}{c}
x^{s}(k) \\
x^{d}(k) \\
x^{i}(k) \\
z_{1}(k) \\
z_{2}(k) \\
\vdots \\
z_{\theta_{\max }}(k)
\end{array}\right], \\
x^{s} \in \mathbb{R}^{n y}, \quad x^{d} \in \mathbb{C}^{n y . n u . n a}, \quad x^{i} \in \mathbb{R}^{n y}, \quad z_{1}, \ldots, z_{\theta_{\max }} \in \mathbb{R}^{n u} \\
x \in \mathbb{C}^{n x}, n x=2 n y+n d+\theta_{\max } \cdot n u, \quad n d=n y . n u . n a,
\end{gathered}
$$

O estado $x^{s}(k)$ corresponde à predição da saída do sistema em estado estacionário, estando relacionado com os modos integradores introduzidos pela descrição incremental que foi proposta. Já os estados $x^{d}(k)$ e $x^{i}(k)$ dizem respeito aos modos estáveis e integradores, respectivamente, do sistema original. A saída expandida $y_{e}$ agrega as saídas físicas do sistema $y$ e os estados $z_{l}(k)$, que armazenam as últimas $\theta_{\max }$ variações na entrada, $z_{l}(k)=\Delta u(k-l)$. Por sua vez, $\theta_{\max }=\max _{i, j} \theta_{i, j}$, ou seja, armazenam-se tantas entradas já implementadas quanto necessário para calcular o valor da saída com maior tempo morto.

Explicitando as matrizes $A, B$ e $C$, tem-se: 


$$
\begin{aligned}
& {\left[\begin{array}{c}
x^{s}(k+1) \\
x^{d}(k+1) \\
x^{i}(k+1) \\
z_{1}(k+1) \\
z_{2}(k+1) \\
\vdots \\
z_{\theta_{\max }}(k+1)
\end{array}\right]=\left[\begin{array}{cccccccc}
I_{n y} & 0 & \Delta t I_{n i}^{*} & B_{1}^{s} & B_{2}^{s} & \ldots & B_{\theta_{\max }-1}^{s} & B_{\theta_{\max }}^{s} \\
0 & F & 0 & B_{1}^{d} & B_{2}^{d} & \ldots & B_{\theta_{\max }}^{d}-1 & B_{\theta_{\max }}^{d} \\
0 & 0 & I_{n i}^{*} & B_{1}^{i} & B_{2}^{i} & \ldots & B_{\theta_{\max }}^{i}-1 & B_{\theta_{\max }^{i}}^{i} \\
0 & 0 & 0 & 0 & 0 & \ldots & 0 & 0 \\
0 & 0 & 0 & I_{n u} & 0 & \ldots & 0 & 0 \\
\vdots & \vdots & \vdots & \vdots & \ddots & \ldots & \vdots & \vdots \\
0 & 0 & 0 & 0 & 0 & \ldots & I_{n u} & 0
\end{array}\right]\left[\begin{array}{c}
x^{s}(k) \\
x^{d}(k) \\
x^{i}(k) \\
z_{1}(k) \\
z_{2}(k) \\
\vdots \\
z_{\theta_{\max }}(k)
\end{array}\right]+\left[\begin{array}{c}
B_{0}^{s} \\
B_{0}^{d} \\
B_{0}^{i} \\
I_{n u} \\
0 \\
\vdots \\
z_{1}(k) \\
\vdots \\
z_{\theta_{\max }}(k)
\end{array}\right]=\left[\begin{array}{ccccccc}
I_{n y} & \Psi & 0 & 0 & 0 & \ldots & 0 \\
0 & 0 & 0 & I_{n u} & 0 & \ldots & 0 \\
0 & 0 & 0 & 0 & I_{n u} & \ldots & 0 \\
\vdots & \vdots & \vdots & \vdots & \vdots & \ddots & \vdots \\
0 & 0 & 0 & 0 & 0 & \ldots & I_{n u}
\end{array}\right]\left[\begin{array}{c}
\Delta \\
x^{s}(k) \\
x^{d}(k) \\
x^{i}(k) \\
z_{1}(k) \\
z_{2}(k) \\
\vdots \\
z_{\theta_{\max }}(k)
\end{array}\right]}
\end{aligned}
$$

As matrizes $A, B$ e $C$ são compostas por diversas submatrizes. Por abuso de notação, representam-se por 0 todas as matrizes nulas, sendo suas dimensões determinadas pelo contexto. $I_{n}$ representa uma matriz identidade de dimensão $n$. Por outro lado, $I_{n y}^{*}$ é uma matriz quadrada de dimensão ny, diagonal, em que as posições correspondentes às saídas integradoras valem 1, enquanto as correspondentes às demais saídas valem 0 .

Se os pólos estáveis do sistema não se repetirem, então a matriz $F$ define a dinâmica dos modos estáveis e é da seguinte forma:

$F=\operatorname{diag}\left(e^{\Delta t \cdot r_{1,1,1}} \cdots e^{\Delta t \cdot r_{1,1, n a}} \cdots e^{\Delta t \cdot r_{1, n u, 1}} \cdots e^{\Delta t \cdot r_{1, n u, n a}} \cdots e^{\Delta t \cdot r_{n y}, 1,1} \cdots e^{\Delta t \cdot r_{n y}, 1, n a} \cdots e^{\Delta t \cdot r_{n y}, n u, 1} \cdots e^{\Delta t \cdot r_{n y}, n u, n a}\right)$ $F \in \mathbb{C}^{n d \times n d}$

As matrizes que de fato relacionam as entradas do sistema aos estados são $B_{l}^{s}, B_{l}^{d}, B_{l}^{i}$, com $l=0, \ldots, \theta_{\max }$. Todas as matrizes $B_{l}^{S}$ e $B_{l}^{i}$ têm $n y$ linhas e $n u$ colunas. Usando a notação $[X]_{i, j}$ para representar o elemento da i-ésima linha e j-ésima coluna de uma matriz $X$, as regras para construção de $B_{l}^{S}$ e $B_{l}^{i}$ são: 
- Se $l \neq \theta_{i, j}$, então $\left[B_{l}^{s}\right]_{i, j}=0 ;\left[B_{l}^{i}\right]_{i, j}=0$

- Caso contrário, $\left[B_{l}^{s}\right]_{i, j}=d_{i, j}^{0}+\Delta t d_{i, j}^{i} ;\left[B_{l}^{i}\right]_{i, j}=d_{i, j}^{i}$

Por fim, a construção de $B_{l}^{d}$ é um pouco mais sutil porque ela tem $n d$ linhas e $n u$ colunas, portanto essa matriz não tem as mesmas dimensões da função de transferência $G(s)$. É necessário estabelecer a correta identificação entre cada elemento de $B_{l}^{d}$ e a entrada e saída correspondentes.

Se não houvesse tempos mortos, então ocorreria apenas o caso $l=0$ e se teria $B_{0}^{d}=D^{d} F N$, sendo:

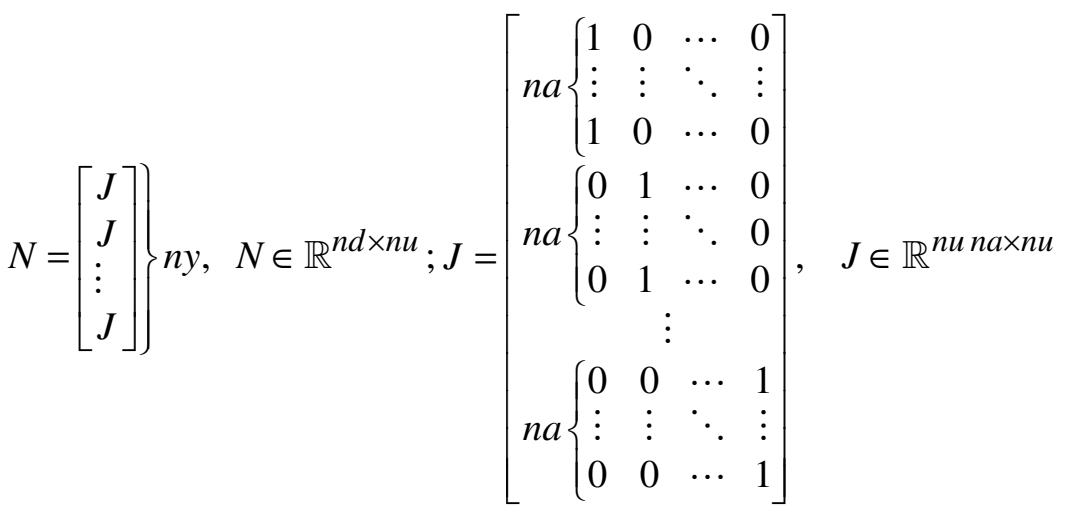

$$
\begin{aligned}
& D^{d}=\operatorname{diag}\left(d_{1,1,1}^{d} \cdots d_{1,1, n a}^{d} \cdots d_{1, n u, 1}^{d} \cdots d_{1, n u, n a}^{d} \cdots d_{n y, 1,1}^{d} \cdots d_{n y, 1, n a}^{d} \cdots d_{n y, n u, 1}^{d} \cdots d_{n y, n u, n a}^{d}\right) \quad D^{d} \in \mathbb{C}^{n d \times n d}
\end{aligned}
$$

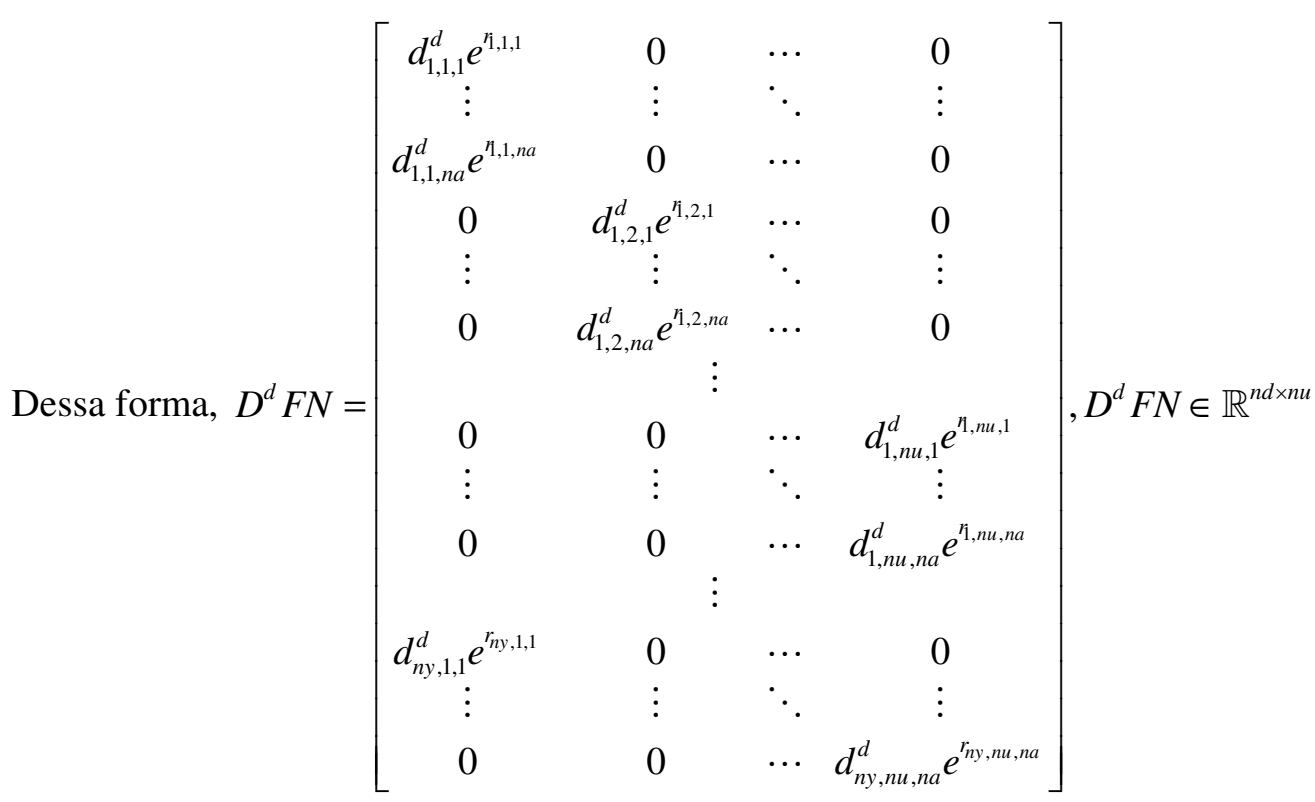


$\mathrm{Na}$ presença de tempos mortos, os elementos de $D^{d} F N$ devem ser distribuídos entre as matrizes $B_{l}^{d}, l=0, \ldots, \theta_{\max }$. Para tanto, cada matriz $B_{l}^{d}$ é uma cópia de $D^{d} F N$ mas os elementos correspondentes às funções de transferência com tempo morto diferente de $l$ são substituídos por zeros. Por exemplo, se o tempo morto da função de transferência relacionando a primeira entrada e a primeira saída é igual a 0 , então $\left[B_{0}^{d}\right]_{1,1}=d_{1,1,1}^{d} e^{\eta_{1,1,1}}$ ao passo que $\left[B_{l}^{d}\right]_{1,1}=0, l \neq 0$.

A relação entre os estados e as saídas é dada por $y(k)=x^{s}(k)+\Psi x^{d}(k)$, sendo

$\Psi=\left[\begin{array}{lll}\Phi & & 0 \\ & \ddots & \\ 0 & & \Phi\end{array}\right], \quad \Psi \in \mathbb{R}^{n y \times n d}, \quad \Phi=\left[\begin{array}{lll}1 & \cdots & 1\end{array}\right], \quad \Phi \in \mathbb{R}^{n u n a}$

\subsubsection{Modelo Contendo Apenas Estados Reais}

A formulação desenvolvida na seção 3.1 compreende algumas matrizes que não são necessariamente reais, como $F$ e $D^{d}$. Conseqüentemente, o estado $x$ pode não ser estritamente real caso o sistema tenha pólos complexos conjugados. A presença de números complexos não-reais causa problemas quando essas matrizes são utilizadas em problemas de LMI, uma vez que os algoritmos atuais lidam apenas com valores estritamente reais.

Considere-se inicialmente um sistema com uma entrada, uma saída sem tempo morto. Admite-se que a função de transferência é de segunda ordem, de maneira que os pólos sejam complexos conjugados:

$$
G(s)=\frac{\alpha+\beta \cdot i}{s+b+\omega \cdot i}+\frac{\alpha-\beta \cdot i}{s+b-\omega \cdot i}
$$

De acordo com (3-4), a resposta desse sistema a um degrau unitário é 


$$
\begin{aligned}
& S(k \Delta t)=d^{0}+d^{d} e^{r k \Delta t}+\bar{d}^{d} e^{r k \Delta t} \\
& d^{0}=\frac{2(\alpha b+\omega \beta)}{b^{2}+\omega^{2}} \\
& d^{d}=\alpha+\beta \cdot i, \quad \bar{d}^{d}=\alpha-\beta \cdot i \\
& r=-b-\omega \cdot i, \quad \bar{r}=-b+\omega \cdot i
\end{aligned}
$$

Expandindo as exponenciais complexas, tem-se:

$$
S(k \Delta t)=d^{0}+2 \alpha e^{-b k \Delta t} \cos (\omega k \Delta t)+2 \beta e^{-b k \Delta t} \sin (\omega k \Delta t)
$$

A representação em espaço de estados desse sistema tem 3 componentes: $x^{s}(k), x_{1}^{d}(k)$ e $x_{2}^{d}(k)$. Na formulação com variáveis complexas, tem-se que a saída é a soma dessas 3 componentes, ou seja, $y(k)=x^{s}(k)+x_{1}^{d}(k)+x_{2}^{d}(k)$. Ao restringir a formulação apenas a variáveis reais, é conveniente definir $y(k)=x^{s}(k)+x_{1}^{d}(k)$, de maneira que a contribuição dos modos estáveis para a saída esteja concentrada apenas em uma das componentes. No entanto, a componente $x_{2}^{d}(k)$ afeta $x_{1}^{d}(k) \mathrm{e}$, indiretamente, também interfere na saída. A matriz de transição A pode ser redefinida através de uma relação de similaridade:

$$
\begin{aligned}
& \text { Caso complexo: } A=\left[\begin{array}{ccc}
1 & 0 & 0 \\
0 & e^{-b \Delta t}(\cos (\omega \Delta t)+i \sin (\omega \Delta t) & 0 \\
0 & 0 & e^{-b \Delta t}(\cos (\omega \Delta t)-i \sin (\omega \Delta t)
\end{array}\right] \\
& \text { Caso real: } A=\left[\begin{array}{ccc}
1 & 0 & 0 \\
0 & e^{-b \Delta t} \cos (\omega \Delta t) & -e^{-b \Delta t} \sin (\omega \Delta t) \\
0 & e^{-b \Delta t} \sin (\omega \Delta t) & e^{-b \Delta t} \cos (\omega \Delta t)
\end{array}\right]
\end{aligned}
$$

Até este ponto, admitiram-se estruturas particulares para as matrizes A e C. Resta calcular as componentes $B_{1}$ e $B_{2}$ da matriz $B$, definidas de acordo com: 


$$
\begin{aligned}
& {\left[\begin{array}{c}
x^{s}(k+1) \\
x_{1}^{d}(k+1) \\
x_{2}^{d}(k+1)
\end{array}\right]=\left[\begin{array}{ccc}
1 & 0 & 0 \\
0 & e^{-b \Delta t} \cos (\omega \Delta t) & -e^{-b \Delta t} \sin (\omega \Delta t) \\
0 & e^{-b \Delta t} \sin (\omega \Delta t) & e^{-b \Delta t} \cos (\omega \Delta t)
\end{array}\right] \cdot\left[\begin{array}{c}
x^{s}(k) \\
x_{1}^{d}(k) \\
x_{2}^{d}(k)
\end{array}\right]+\left[\begin{array}{c}
d^{0} \\
B_{1} \\
B_{2}
\end{array}\right] \cdot \Delta u(k)} \\
& y(k)=\left[\begin{array}{lll}
1 & 1 & 0
\end{array}\right] \cdot\left[\begin{array}{c}
x^{s}(k) \\
x_{1}^{d}(k) \\
x_{2}^{d}(k)
\end{array}\right]
\end{aligned}
$$

Este cálculo pode ser feito considerando que a predição da resposta ao degrau dada em (3-11) deve ser igual à predição que seria obtida usando (3-13). Considera-se, para isso, que um estado inicialmente na origem $(x(0)=0)$ seja submetido a um degrau unitário no instante inicial $(\Delta u(0)=1, \Delta u(k)=0$ se $k>0)$.

De acordo com (3-11), a saída no instante $k=1$ é:

$$
y(1)=S(\Delta t)=d^{0}+2 \alpha e^{-b \Delta t} \cos (\omega \Delta t)+2 \beta e^{-b \Delta t} \sin (\omega \Delta t)
$$

Por outro lado, segundo (3-13), tem-se:

$$
\begin{aligned}
y(1) & =x^{s}(1)+x_{1}^{d}(1) \\
& =d^{0} \Delta u(0)+\cos (\omega \Delta t) e^{-b \Delta t} x_{1}^{d}(0)+\sin (\omega \Delta t) e^{-b \Delta t} x_{2}^{d}(0)+B_{1} \Delta u(0) \\
& =d^{0}+B_{1}
\end{aligned}
$$

Como ambas as predições devem ser iguais, tem-se:

$$
\begin{aligned}
& d^{0}+2 \alpha e^{-b \Delta t} \cos (\omega \Delta t)+2 \beta e^{-b \Delta t} \sin (\omega \Delta t)=d^{0}+B_{1} \\
& B_{1}=2 \alpha e^{-b \Delta t} \cos (\omega \Delta t)+2 \beta e^{-b \Delta t} \sin (\omega \Delta t)
\end{aligned}
$$

Procede-se de maneira análoga para determinar $B_{2}$, primeiramente calculando a predição com a resposta ao degrau e depois com a formulação em espaço de estados:

$$
y(2)=S(2 \Delta t)=d^{0}+2 \alpha e^{-2 b \Delta t} \cos (2 \omega \Delta t)+2 \beta e^{-2 b \Delta t} \sin (2 \omega \Delta t)
$$




$$
\begin{aligned}
y(2) & =x^{s}(2)+x_{1}^{d}(2) \\
& =d^{0}+\cos (\omega \Delta t) e^{-b \Delta t} x_{1}^{d}(1)-\sin (\omega \Delta t) e^{-b \Delta t} x_{2}^{d}(1)
\end{aligned}
$$

Como os estados partem da origem e o degrau é unitário, então $x_{1}^{d}(1)=B_{1}, x_{2}^{d}(1)=B_{2}$ :

$$
y(2)=d^{0}+\cos (\omega \Delta t) e^{-b \Delta t} B_{1}-\sin (\omega \Delta t) e^{-b \Delta t} B_{2}
$$

Igualando-se as predições (3-17) e (3-19):

$$
\begin{aligned}
& d^{0}+2 \alpha e^{-2 b \Delta t} \cos (2 \omega \Delta t)+2 \beta e^{-2 b \Delta t} \sin (2 \omega \Delta t)= \\
& d^{0}+\cos (\omega \Delta t) e^{-b \Delta t} B_{1}-\sin (\omega \Delta t) e^{-b \Delta t} B_{2}
\end{aligned}
$$

Utilizando no primeiro membro as relações trigonométricas de arco duplo e no segundo o valor previamente calculado de $B_{1}$, tem-se:

$$
\begin{aligned}
& d^{0}+2 \alpha e^{-2 b \Delta t}\left(\cos ^{2}(\omega \Delta t)-\sin ^{2}(\omega \Delta t)\right)+2 \beta e^{-2 b \Delta t}(2 \cos (\omega \Delta t) \sin (\omega \Delta t))= \\
& d^{0}+\cos (\omega \Delta t) e^{-b \Delta t}\left(2 \alpha e^{-b \Delta t} \cos (\omega \Delta t)+2 \beta e^{-b \Delta t} \sin (\omega \Delta t)\right)-\sin (\omega \Delta t) e^{-b \Delta t} B_{2}
\end{aligned}
$$

Realizando as simplificações possíveis, conclui-se que:

$$
B_{2}=2 \alpha e^{-b \Delta t} \sin (\omega \Delta t)-2 \beta e^{-b \Delta t} \cos (\omega \Delta t)
$$

Portanto, um modelo possível para o caso de um sistema com uma entrada, uma saída e pólos conjugados complexos é:

$$
\begin{aligned}
& {\left[\begin{array}{l}
x^{s}(k+1) \\
x_{1}^{d}(k+1) \\
x_{2}^{d}(k+1)
\end{array}\right]=\left[\begin{array}{ccc}
1 & 0 & 0 \\
0 & e^{-b \Delta t} \cos (\omega \Delta t) & -e^{-b \Delta t} \sin (\omega \Delta t) \\
0 & e^{-b \Delta t} \sin (\omega \Delta t) & e^{-b \Delta t} \cos (\omega \Delta t)
\end{array}\right]\left[\begin{array}{c}
x^{s}(k) \\
x_{1}^{d}(k) \\
x_{2}^{d}(k)
\end{array}\right]+\left[\begin{array}{c}
d^{0} \\
2 e^{-b \Delta t}(\alpha \cos (\omega \Delta t)+\beta \sin (\omega \Delta t)) \\
2 e^{-b \Delta t}(-\beta \cos (\omega \Delta t)+\alpha \sin (\omega \Delta t))
\end{array}\right] \cdot \Delta u(k)} \\
& y(k)=\left[\begin{array}{lll}
1 & 1 & 0
\end{array}\right] \cdot\left[\begin{array}{c}
x^{s}(k) \\
x_{1}^{d}(k) \\
x_{2}^{d}(k)
\end{array}\right]
\end{aligned}
$$


Generalizado esse resultado para o caso de um sistema com ny saídas e $n u$ entradas, é útil notar que o bloco composto pelas matrizes $B_{1}, B_{2}$ correspondia, na formulação (3-8) a $D^{d} F N$. A partir da definição modificada de F para não utilizar variáveis complexas, feita em (3-12), pode-se calcular qual o valor apropriado para $D^{d}$ :

$$
\begin{aligned}
& D^{d} F N=\left[\begin{array}{l}
B_{1} \\
B_{2}
\end{array}\right] \\
& D^{d}\left[\begin{array}{cc}
e^{-b \Delta t} \cos (\omega \Delta t) & -e^{-b \Delta t} \sin (\omega \Delta t) \\
e^{-b \Delta t} \sin (\omega \Delta t) & e^{-b \Delta t} \cos (\omega \Delta t)
\end{array}\right]\left[\begin{array}{l}
1 \\
1
\end{array}\right]=\left[\begin{array}{c}
2 e^{-b \Delta t}(\alpha \cos (\omega \Delta t)+\beta \sin (\omega \Delta t)) \\
2 e^{-b \Delta t}(-\beta \cos (\omega \Delta t)+\alpha \sin (\omega \Delta t))
\end{array}\right]
\end{aligned}
$$

Admitindo-se que $D^{d}$ é uma matriz 2 por 2 qualquer, podem-se calcular cada uma de suas componentes:

$$
\begin{aligned}
& {\left[\begin{array}{ll}
a_{11} & a_{12} \\
a_{21} & a_{22}
\end{array}\right]\left[\begin{array}{ll}
e^{-b \Delta t} \cos (\omega \Delta t) & -e^{-b \Delta t} \sin (\omega \Delta t) \\
e^{-b \Delta t} \sin (\omega \Delta t) & e^{-b \Delta t} \cos (\omega \Delta t)
\end{array}\right]\left[\begin{array}{l}
1 \\
1
\end{array}\right]=\left[\begin{array}{c}
2 e^{-b \Delta t}(\alpha \cos (\omega \Delta t)+\beta \sin (\omega \Delta t)) \\
2 e^{-b \Delta t}(-\beta \cos (\omega \Delta t)+\alpha \sin (\omega \Delta t))
\end{array}\right]} \\
& a_{11}=\alpha-\beta \\
& a_{12}=\alpha+\beta \\
& a_{21}=-\alpha-\beta \\
& a_{22}=\alpha-\beta
\end{aligned}
$$

É importante ressaltar que a matriz $D^{d}$ também perde sua estrutura diagonal mas, ao contrário de $F$, ela não é obtida por um argumento de similaridade de matrizes.

Em resumo, para transformar a representação em estados estritamente reais, três modificações devem ser feitas com relação aos elementos correspondentes aos estados complexos conjugados:

- Substituir os elementos da diagonal de F por blocos 2 x 2:

$$
\operatorname{De}\left[\begin{array}{cc}
e^{\Delta t \cdot r_{i, j, 1}} & 0 \\
0 & e^{\Delta t \cdot \bar{r}_{i, j, 1}}
\end{array}\right] \text { para }\left[\begin{array}{cc}
e^{-b \cdot \Delta t} \cos (\omega \Delta t) & -e^{-b \cdot \Delta t} \sin (\omega \Delta t) \\
e^{-b \cdot \Delta t} \sin (\omega \Delta t) & e^{-b \cdot \Delta t} \cos (\omega \Delta t)
\end{array}\right]
$$


- Substituir os elementos da diagonal de $D^{d}$ por blocos 2 × 2 :

$\operatorname{De}\left[\begin{array}{cc}d^{d} & 0 \\ 0 & \bar{d}^{d}\end{array}\right]$ para $\left[\begin{array}{cc}\alpha-\beta & \alpha+\beta \\ -\alpha-\beta & \alpha-\beta\end{array}\right]$

- Substituir os elementos de $\Phi$ relativos à leitura dos estados associados a pólos complexos:

De $\left[\begin{array}{ll}1 & 1\end{array}\right]$ para $\left[\begin{array}{ll}1 & 0\end{array}\right]$

\subsection{MODELO CONSIDERANDO DISTÚRBIOS MEDIDOS}

A formulação desenvolvida na seção 3.1 pressupõe que o sistema a ser controlado possui $n y$ saídas e $n u$ entradas. Por hipótese, considera-se que todas as entradas do sistema são graus de liberdade do controlador, ou seja, o controlador calcula quais as melhores ações a implementar em cada instante.

Contudo, na maior parte dos processos industriais reais há entradas que não podem ser manipuladas. Caso essas demais entradas sejam mensuráveis, é possível generalizar o modelo da seção 3.1 para incluir esses distúrbios medidos. Mantendo a hipótese de comportamento linear do sistema, pode-se propor um modelo com a seguinte estrutura:

$$
\begin{aligned}
& x(k+1)=A \cdot x(k)+B \cdot \Delta u(k)+B w \cdot \Delta w(k) \\
& y_{e}(k)=C \cdot x(k)
\end{aligned}
$$

Neste caso, $\Delta w(k) \in \mathbb{R}^{n w}$, sendo $n w$ o número de distúrbios medidos. Para manter a coerência com a representação das demais entradas, o vetor de distúrbios medidos também está escrito em termos incrementais.

Tanto o estado $x$ quanto as matrizes $A, B$ e $C$ devem ser redefinidos em relação à formulação (3-31). Além disso, é necessário explicitar qual a forma esperada da matriz $B w$, que relaciona os distúrbios medidos e o estado. 
Da mesma maneira que se supôs conhecida a matriz $G(s)$, contendo as funções de transferência entre entradas e saídas, admite-se a existência de $G^{w}(s)$, relacionando saídas e distúrbios medidos:

$$
G^{w}(s)=\left[\begin{array}{ccc}
G_{1,1}^{w}(s) & \cdots & G_{1, n w}^{w}(s) \\
\vdots & \ddots & \vdots \\
G_{n y, 1}^{w}(s) & \cdots & G_{n y, n w}^{w}(s)
\end{array}\right]
$$

As funções de transferência $G_{i, j}^{w}(s)$ também são supostas de ordem na e podem apresentar tempos mortos:

$$
G_{i, j}^{w}(s)=\frac{b_{i, j, 0}^{w}+b_{i, j, 1}^{w} s+\cdots+b_{i, j, n b}^{w} s^{n b}}{s\left(s-r_{i, j, 1}^{w}\right)\left(s-r_{i, j, 2}^{w}\right) \cdots\left(s-r_{i, j, n a}^{w}\right)} e^{-\tau_{i, j} s}
$$

Analogamente ao realizado com as relações entrada manipulada-saída, a expansão em frações parciais e a resposta ao degrau das funções de transferência dos distúrbios são:

$$
\begin{gathered}
S_{i, j}^{w}(s)=\frac{G_{i, j}^{w}(s)}{s}=\frac{d w_{i, j}^{0}}{s} e^{-\tau_{i, j} s}+\frac{d w_{i, j, 1}^{p}}{s-r_{i, j, 1}^{w}} e^{-\tau_{i, j} s}+\cdots+\frac{d w_{i, j, n a}^{p}}{s-r_{i, j, n a}^{w}} e^{-\tau_{i, j} s}+\frac{d w_{i, j}^{i}}{s^{2}} e^{-\tau_{i, j} s} \\
S_{i, j}^{w}(k \Delta t)=\left(d w_{i, j}^{0}+d w_{i, j, 1}^{p} e^{r_{i, j, k}^{w} k \Delta t-\tau_{i, j}}+\cdots+d w_{i, j, n a}^{p} e^{r_{i, j, n a}^{w} k \Delta t-\tau_{i, j}}+d w_{i, j}^{i}\left(k \Delta t-\tau_{i, j}\right)\right) \\
\cdot U\left(\Delta t-\tau_{i, j}\right)
\end{gathered}
$$

O estado nessa nova formulação contém fundamentalmente duas diferenças. Em primeiro lugar, definem-se componentes $x^{w}$ para calcular a evolução dos modos estáveis das funções de transferência dos distúrbios medidos. Além disso, devido à presença de tempos mortos, é necessário armazenar as últimas $\tau_{\max }$ variações dos distúrbios, com $\tau_{\max }=\max _{i, j} \tau_{i, j}$. Nota-se que, em geral, $\tau_{\max } \neq \theta_{\max }$. Por analogia ao caso sem distúrbios, define-se $z_{l}^{w}(k)=\Delta w(k-l)$. O estado da formulação com distúrbios é portanto dado por: 


$$
x(k)=\left[\begin{array}{c}
x^{s}(k) \\
x^{d}(k) \\
x^{w}(k) \\
x^{i}(k) \\
z_{1}(k) \\
\vdots \\
z_{\theta_{\max }}(k) \\
z_{1}^{w}(k) \\
\vdots \\
z_{\tau_{\max }}^{w}(k)
\end{array}\right],
$$

Para redefinir a matriz A, nota-se que a componente $x^{s}$ pode ser afetada pelos distúrbios, vez que estes alteram o estado estacionário para o qual o sistema convergiria se não fosse mais excitado. Da mesma maneira, $x^{i}$ também pode variar com os distúrbios: considerando por exemplo um tanque em que um dos distúrbios é uma vazão de entrada, é fácil perceber que uma alteração nesta vazão alterará a velocidade com o que o tanque é preenchido.

Por outro lado, a componente $x^{d}$ relaciona apenas as entradas com as saídas, não sendo alterada com a inclusão de distúrbios. De modo contrário, a componente $x^{w}$ varia apenas com os distúrbios mas não com as entradas.

Por fim, as componentes $z_{l}$ e $z_{l}^{w}$ devem variar no tempo de modo a guardar as últimas variações das entradas e distúrbios.

Com relação à expressão das saídas, o fato do sistema ser linear implica que as saídas no caso com distúrbio são iguais às saídas sem distúrbios acrescidas das contribuições desses distúrbios. Logo, as matrizes de evolução do estado em função do tempo e do cálculo das saídas são dadas por: 
(3-31)

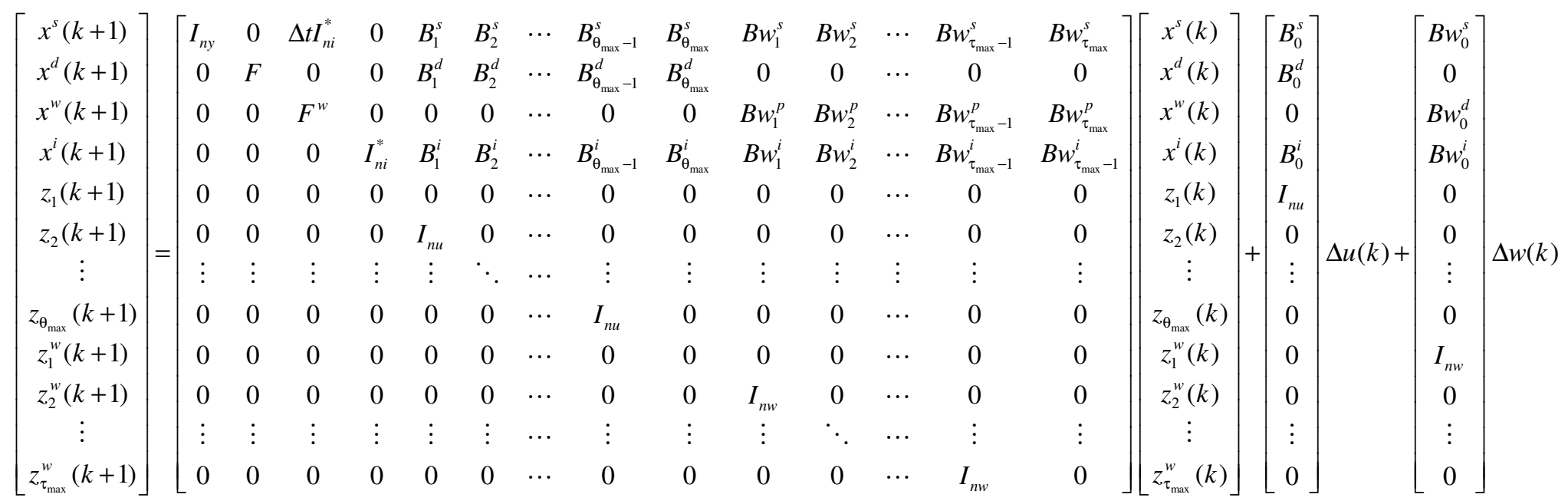

$$
\begin{aligned}
& {\left[\begin{array}{c}
y(k) \\
z_{1}(k) \\
z_{2}(k) \\
\vdots \\
z_{\theta_{\max }}(k) \\
z_{1}^{w}(k) \\
z_{2}^{w}(k) \\
\vdots \\
z_{\tau_{\max }^{w}}^{w}(k)
\end{array}\right]=\left[\begin{array}{cccccccccccc}
I_{n y} & \Psi & \Psi^{w} & 0 & 0 & 0 & \cdots & 0 & 0 & 0 & \cdots & 0 \\
0 & 0 & 0 & 0 & I_{n u} & 0 & \cdots & 0 & 0 & 0 & \cdots & 0 \\
0 & 0 & 0 & 0 & 0 & I_{n u} & \cdots & 0 & 0 & 0 & \cdots & 0 \\
\vdots & \vdots & \vdots & \vdots & \vdots & \vdots & \ddots & \vdots & \vdots & \vdots & \vdots & \vdots \\
0 & 0 & 0 & 0 & 0 & 0 & \cdots & I_{n u} & 0 & 0 & \cdots & 0 \\
0 & 0 & 0 & 0 & 0 & 0 & \cdots & 0 & I_{n w} & 0 & \cdots & 0 \\
0 & 0 & 0 & 0 & 0 & 0 & \cdots & 0 & 0 & I_{n w} & \cdots & 0 \\
\vdots & \vdots & \vdots & \vdots & \vdots & \vdots & \cdots & \vdots & \vdots & \vdots & \ddots & \vdots \\
0 & 0 & 0 & 0 & 0 & 0 & \cdots & 0 & 0 & 0 & \cdots & I_{n w}
\end{array}\right]\left[\begin{array}{c}
x^{s}(k) \\
x^{d}(k) \\
x^{w}(k) \\
x^{i}(k) \\
z_{1}(k) \\
z_{2}(k) \\
\vdots \\
z_{\theta_{\max }}(k) \\
z_{1}^{w}(k) \\
z_{2}^{w}(k) \\
\vdots \\
z_{\tau_{\max }}^{w}(k)
\end{array}\right]}
\end{aligned}
$$


A forma de cálculo das matrizes auxiliares que aparecem em (3-31) é em grande parte idêntica ao que se fez na seção 3.1. Primeiramente, assim como $F$ é responsável pela evolução dos modos estáveis das relações entrada-saída, tem-se que $F^{w}$ define a dinâmica dos modos estáveis das relações distúrbio-saída:

$$
\begin{aligned}
& F^{w}=\operatorname{diag}\left(e^{\Delta t \cdot r_{1,1,1}^{w}} \cdots e^{\Delta t \cdot r_{1,1, n a}^{w}} \cdots e^{\Delta t \cdot 1, n w, 1} \cdots e^{\Delta t \cdot r_{1, n w, n a}^{w}} \cdots e^{\Delta t \cdot r_{n y, 1,1}^{w}} \cdots e^{\Delta t \cdot r_{n y, 1, n a}^{w}} \cdots e^{\Delta t \cdot r_{n y, n w, 1}^{w}} \cdots e^{\Delta t \cdot r_{n y, n w, n a}^{w}}\right) \\
& F^{w} \in \mathbb{C}^{n p \times n n} .
\end{aligned}
$$

As matrizes $B w_{l}^{s}$ e $B w_{l}^{i}\left(l=0, \cdots, \tau_{\max }\right)$ são análogas a $B_{l}^{s}$ e $B_{l}^{i}$, ou seja,

$$
\begin{aligned}
& \text { - Se } l \neq \tau_{i, j} \text {, então }\left[B w_{l}^{s}\right]_{i, j}=0 ;\left[B w_{l}^{i}\right]_{i, j}=0 \\
& \text { - Caso contrário, }\left[B w_{l}^{s}\right]_{i, j}=d w_{i, j}^{0}+\Delta t d w_{i, j}^{i} ;\left[B w_{l}^{i}\right]_{i, j}=d w_{i, j}^{i}
\end{aligned}
$$

Da mesma maneira que o cálculo de $B_{l}^{d}$ é iniciado com o produto $D^{d} F N$, para determinar $B w_{l}^{p}$ é necessário multiplicar $D w^{p} F^{w} N^{w}$, sendo:

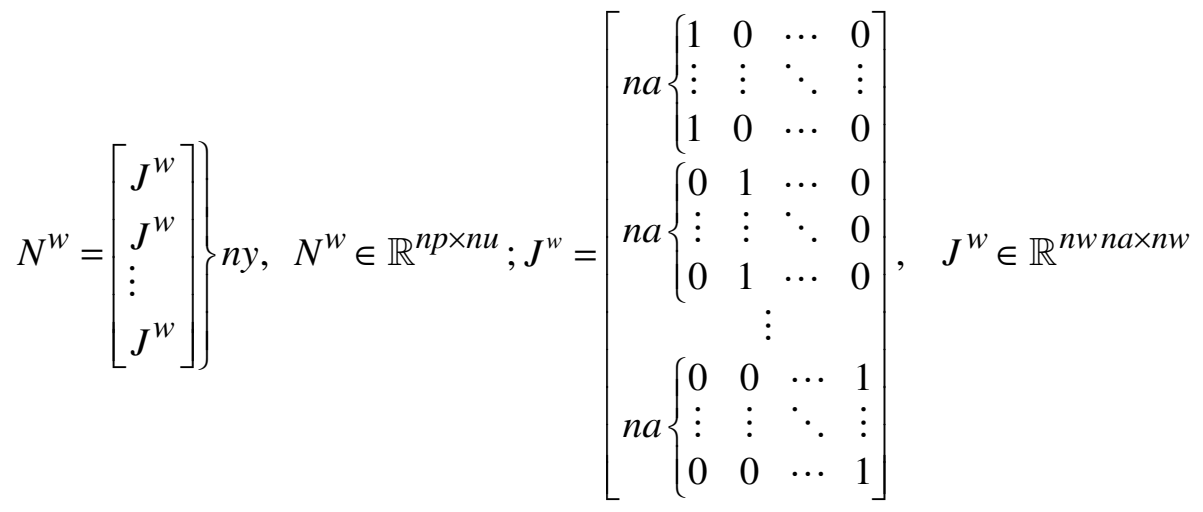

$$
\begin{aligned}
& D w^{p}=\operatorname{diag}\left(d w_{1,1,1}^{p} \cdots d w_{1,1, n a}^{p} \cdots d w_{1, n w, 1}^{p} \cdots d w_{1, n w, n a}^{p} \cdots d w_{n y, 1,1}^{p} \cdots d w_{n y, 1, n a}^{p} \cdots d w_{n y, n w, 1}^{p} \cdots d w_{n y, n w, n a}^{p}\right) \\
& D w^{p} \in \mathbb{C}^{n p \times n p}
\end{aligned}
$$

Considerando os diferentes tempos mortos, as matrizes $B w_{l}^{p}$ são obtidas a partir de $D w^{p} F^{w} N^{w}$ como explicado na seção 3.1 .

Finalmente, as saídas são calculadas a partir de $y(k)=x^{s}(k)+\Psi x^{d}(k)+\Psi^{w} x^{w}(k)$, sendo 
$\Psi^{w}=\left[\begin{array}{ccc}\Phi^{w} & & 0 \\ & \ddots & \\ 0 & & \Phi^{w}\end{array}\right], \quad \Psi^{w} \in \mathbb{R}^{n y \times n p}, \quad \Phi^{w}=\left[\begin{array}{lll}1 & \cdots & 1\end{array}\right], \quad \Phi \in \mathbb{R}^{n w n a}$

Caso seja necessário trabalhar com valores estritamente reais, então as matrizes $D w^{d} \mathrm{e}$ $F^{w}$ passam a ser bloco-diagonais como deduzido na seção 3.1.1. Conseqüentemente, para cada par de pólos complexos conjugados, $\Psi^{w}$ terá uma linha composta normalmente por $\Phi^{w}$ e outra apenas com zeros.

\subsection{DETECTABILIDADE DOS MODELOS PROPOSTOS}

Define-se na literatura de sistemas lineares (Hespanha, 2009) que um sistema é dito observável se para qualquer estado inicial $x(0)$ existir um tempo finito $t_{1}$ tal que a partir das entradas e saídas no intervalo $\left[0, t_{1}\right]$ seja possível determinar $x(0)$. Um sistema é detectável se for observável ou se os estados não observáveis convergirem para 0 , ou seja, um sistema detectável pode ser decomposto da seguinte forma:

$$
\left[\begin{array}{c}
x_{o}(k+1) \\
x_{n o}(k+1)
\end{array}\right]=\left[\begin{array}{cc}
A_{o} & 0 \\
A_{21} & A_{n o}
\end{array}\right]\left[\begin{array}{c}
x_{o}(k) \\
x_{n o}(k)
\end{array}\right]
$$

em que $x_{o}$ corresponde aos estados observáveis, $x_{n o}$ corresponde aos estados não-observáveis e a matriz $A_{n o}$ é estável (o módulo de todos os autovalores é estritamente menor do que 1).

A matriz de observabilidade $\mathfrak{O}$ é definida por:

$$
\mathfrak{O}=\left[\begin{array}{c}
C \\
C A \\
C A^{2} \\
\vdots \\
C A^{n-1}
\end{array}\right]
$$


em que $n$ é a dimensão do estado. Um dos métodos possíveis para checar a observabilidade de um sistema consiste no cálculo do posto da matriz $\mathfrak{O}$. Se ele for igual ao número de colunas (posto completo de colunas), então o sistema é observável.

O modelo apresentado em (3-8) apresenta uma definição pouco comum para a matriz $C$, visando à incorporação dos estados $z_{l}$, correspondentes às entradas $\Delta u(k-l)$, às saídas do sistema. O modelo assim definido torna-se detectável. Em uma formulação anterior de MPC com tempo morto (Carrapiço, 2004) pode-se mostrar que a detectabilidade não é alcançada quando há tempos mortos diferentes. A detectabilidade da presente formulação pode ser vista numericamente a partir do seguinte exemplo extraído de Carrapiço (2004):

$$
G(s)=\left[\begin{array}{ll}
G_{1,1}(s) & G_{1,2}(s) \\
G_{2,1}(s) & G_{2,2}(s)
\end{array}\right]=\left[\begin{array}{cc}
\frac{-0.19 e^{-s}}{s} & \frac{-1.7}{s} \\
\frac{-0.763}{31.8 s+1} & \frac{0.235}{s}
\end{array}\right]
$$

Com o modelo (3-8) obtêm-se:

$$
A=\left[\begin{array}{cccccccccc}
1 & 0 & 0 & 0 & 0 & 0 & 1 & 0 & -0.19 & 0 \\
0 & 1 & 0 & 0 & 0 & 0 & 0 & 1 & 0 & 0 \\
0 & 0 & 0 & 0 & 0 & 0 & 0 & 0 & 0 & 0 \\
0 & 0 & 0 & 0 & 0 & 0 & 0 & 0 & 0 & 0 \\
0 & 0 & 0 & 0 & 0.969 & 0 & 0 & 0 & 0 & 0 \\
0 & 0 & 0 & 0 & 0 & 0 & 0 & 0 & 0 & 0 \\
0 & 0 & 0 & 0 & 0 & 0 & 1 & 0 & -0.19 & 0 \\
0 & 0 & 0 & 0 & 0 & 0 & 0 & 1 & 0 & 0 \\
0 & 0 & 0 & 0 & 0 & 0 & 0 & 0 & 0 & 0 \\
0 & 0 & 0 & 0 & 0 & 0 & 0 & 0 & 0 & 0
\end{array}\right] ; C=\left[\begin{array}{cccccccccc}
1 & 0 & 1 & 1 & 0 & 0 & 0 & 0 & 0 & 0 \\
0 & 1 & 0 & 0 & 1 & 1 & 0 & 0 & 0 & 0 \\
& & & & & & & & &
\end{array}\right]
$$

Portanto, $\mathfrak{O}$ é dada por: 


$$
\mathfrak{O}=\left[\begin{array}{cccccccccc}
1 & 0 & 1 & 1 & 0 & 0 & 0 & 0 & 0 & 0 \\
0 & 1 & 0 & 0 & 1 & 1 & 0 & 0 & 0 & 0 \\
1 & 0 & 0 & 0 & 0 & 0 & 1 & 0 & -0.19 & 0 \\
0 & 1 & 0 & 0 & 0.969 & 0 & 0 & 1 & 0 & 0 \\
1 & 0 & 0 & 0 & 0 & 0 & 2 & 0 & -0.38 & 0 \\
0 & 1 & 0 & 0 & 0.939 & 0 & 0 & 2 & 0 & 0 \\
1 & 0 & 0 & 0 & 0 & 0 & 3 & 0 & -0.57 & 0 \\
0 & 1 & 0 & 0 & 0.91 & 0 & 0 & 3 & 0 & 0 \\
1 & 0 & 0 & 0 & 0 & 0 & 4 & 0 & -0.76 & 0 \\
0 & 1 & 0 & 0 & 0.8818 & 0 & 0 & 4 & 0 & 0 \\
1 & 0 & 0 & 0 & 0 & 0 & 5 & 0 & -0.95 & 0 \\
0 & 1 & 0 & 0 & 0.8545 & 0 & 0 & 5 & 0 & 0 \\
1 & 0 & 0 & 0 & 0 & 0 & 6 & 0 & -1.14 & 0 \\
0 & 1 & 0 & 0 & 0.8281 & 0 & 0 & 6 & 0 & 0 \\
1 & 0 & 0 & 0 & 0 & 0 & 7 & 0 & -1.33 & 0 \\
0 & 1 & 0 & 0 & 0.8024 & 0 & 0 & 7 & 0 & 0 \\
1 & 0 & 0 & 0 & 0 & 0 & 8 & 0 & -1.52 & 0 \\
0 & 1 & 0 & 0 & 0.7776 & 0 & 0 & 8 & 0 & 0 \\
1 & 0 & 0 & 0 & 0 & 0 & 9 & 0 & -1.71 & 0 \\
0 & 1 & 0 & 0 & 0.7535 & 0 & 0 & 9 & 0 & 0 \\
& & & & & & & & &
\end{array}\right]
$$

O posto dessa matriz é 7, enquanto o número total de estados é 10. Logo, o sistema não é observável. No entanto, ele é detectável como pode ser visto aplicando-se o critério de Popov-Belevitch-Hautus, o qual pode ser enunciado da seguinte maneira (Hespanha, 2009): um sistema é detectável se e somente se a matriz $\left[\begin{array}{c}A-\lambda I \\ C\end{array}\right]$ tem posto pleno de colunas para qualquer $\lambda \in \mathbb{C},|\lambda| \geq 1$. Aplicando-se esse teste para $\lambda=1$ obtém-se uma matriz com 14 linhas, 10 colunas e com posto igual a 10, confirmando a detectabilidade.

A interpretação do conceito de detectabilidade é que, apesar de alguns estados não serem observáveis, eles convergem para 0 após um tempo suficientemente longo. No caso do sistema deste exemplo, os três estados não-observáveis correspondem a três linhas da matriz A cujos elementos são todos iguais a 0. Conseqüentemente, estes estados permanecem na origem durante toda a evolução do sistema. Logo, a representação proposta é de fato 
detectável, garantindo a existência de um observador de estados em que as estimativas convirjam para os valores reais.

\subsection{FORMULAÇÃO DO PROBLEMA EM 1 PASSO}

Um controlador do tipo MPC permite a inclusão natural de restrições relativas às condições operacionais de qualquer sistema. Devido a limitações dos atuadores e mesmo a limitações físicas das instalações, é costumeiro haver limites sobre as ações de controle e sobre as entradas que são admissíveis em uma planta real:

$$
\begin{gathered}
-\Delta u_{\max } \leq \Delta u(k+j \mid k) \leq \Delta u_{\max }, j=0,1, \ldots, m-1 \\
u_{\min } \leq u(k+j \mid k) \leq u_{\max }, j \in \mathbb{N}
\end{gathered}
$$

O controlador proposto considera a existência valores meta (targets) para as entradas, designados por $u_{d e s}$. Para assegurar a viabilidade do problema de otimização resolvido pelo controlador em cada instante de amostragem, não se impõe exatamente o valor das entradas ao final do horizonte de controle, mas sim através de uma restrição relaxada:

$$
u(k+m-1 \mid k)-u_{d e s}-\delta_{u, k}=0
$$

A variável de folga (slack) $\delta_{u, k}$ é, por definição, irrestrita e assim garante-se a viabilidade de (3-37) em todas as circunstâncias. Como é feito normalmente, penaliza-se fortemente o uso dessa variável de folga na função objetivo de modo a desfavorecer soluções em que $\delta_{u, k} \neq 0$. Além disso, ao contrário da maior parte de formulações de MPC, não será fixado um valor de set-point para as saídas. Esse conceito será substituído por uma zona dentro da qual as saídas devem preferencialmente estar contidas. O valor dos set-points, $y_{s p, k}$, deixa, portanto, de ser um parâmetro fornecido pela camada de otimização e passa a ser uma variável manipulada do controlador. A restrição que deve ser imposta a esse set-point é a própria definição da zona, expressa na equação (3-38).

$$
y_{\min } \leq y_{s p, k} \leq y_{\max }
$$


Deseja-se que o valor previsto para as saídas, após o efeito de todas as ações de controle, seja igual ao set-point. Como isso nem sempre é possível após um número finito de passos, adotase um procedimento análogo ao que é feito com os targets das entradas, ou seja, são incluídas variáveis de folga $\delta_{y, k}$ de modo a garantir a viabilidade dos problemas. Por fim, como o sistema possui tempos mortos, é preciso esperar $m+\theta_{\max }$ intervalos para que a última ação de controle afete a saída com maior tempo morto. Assim, a restrição deve ser formulada conforme (3-39):

$$
x^{s}\left(k+m+\theta_{\max } \mid k\right)-y_{s p, k}-\delta_{y, k}=0
$$

Como o controlador proposto pretende lidar com a estabilização de sistemas em que algumas saídas são integradoras, é necessário incluir explicitamente uma restrição para evitar que os estados relacionados aos modos integradores divirjam. Isso pode ser feito impondo-se que esses modos devem estar zerados quando cessar a influência das ações de controle, ou seja, $m+\theta_{\max }$ intervalos após o instante atual. Contudo, novamente tem-se a situação em que esse objetivo pode não ser factível para todas as condições de operação e assim é preferível formular esta restrição de maneira relaxada através da variável de folga $\delta_{i, k}$ :

$$
x^{i}\left(k+m+\theta_{\max } \mid k\right)-\delta_{i, k}=0
$$

Uma função objetivo adequada para atingir os propósitos expostos anteriormente inclui penalizações sobre:

- desvios entre as saídas previstas e seus respectivos set-points

- desvios entre as entradas calculadas e seus respectivos targets

- amplitude das ações de controle, permitindo regular a intensidade das movimentações dos atuadores através de um parâmetro de sintonia (fator de supressão)

- valor de qualquer uma das variáveis de folga 
Escrevendo esses termos em forma de funções quadráticas, chega-se à seguinte função objetivo:

$$
\begin{aligned}
& \min _{\Delta u_{k}, y_{s p, k}, \delta_{y, k}, \delta_{i, k}, \delta_{u, k}} V_{1, k}=\sum_{j=0}^{\infty}\left\|y(k+j / k)-y_{s p, k}-\delta_{y, k}\right\|_{Q_{y}}^{2}+\sum_{j=0}^{\infty}\left\|u(k+j / k)-u_{d e s}-\delta_{u, k}\right\|_{Q_{u}}^{2} \\
& +\sum_{j=0}^{m-1}\|\Delta u(k+j / k)\|_{R}^{2}+\left\|\delta_{y, k}\right\|_{S_{y}}^{2}+\left\|\delta_{u, k}\right\|_{S_{u}}^{2}+\left\|\delta_{i, k}\right\|_{S_{i}}^{2}
\end{aligned}
$$

A notação $\|\cdot\|_{X}$ indica a norma euclidiana do vetor $\cdot$ ponderada pela matriz simétrica positiva definida $X$.

Para tornar mais sucinta a lista de variáveis de decisão, as ações de controle $\Delta u(k / k), \ldots, \Delta u(k+m-1 / k)$ foram agrupadas no vetor $\Delta u_{k}$ conforme a definição a seguir:

$$
\Delta u_{k}=\left[\begin{array}{c}
\Delta u(k \mid k) \\
\vdots \\
\Delta u(k+m-1 \mid k)
\end{array}\right]
$$

\subsection{FORMULAÇÃO DO PROBLEMA EM 2 PASSOS}

É conveniente para a demonstração de estabilidade do controlador proposto dividir o problema de otimização exposto anteriormente em dois passos: no primeiro deles, tenta-se zerar os modos integradores. O valor calculado para as ações de controle é então usado como restrição sobre as mesmas ações de controle num segundo problema, em que o objetivo é levar as entradas aos seus targets e as saídas às suas faixas.

$\mathrm{O}$ primeiro problema, doravante citado como $\mathrm{P} 2 \mathrm{a}$, pode ser escrito da seguinte forma:

$$
\min _{\Delta u_{k}^{a}} V_{2 a, k}=\left\|x^{i}\left(k+m+\theta_{\max } \mid k\right)\right\|_{Q_{i}}^{2}+\left\|\Delta u_{k}^{a}\right\|_{\bar{R}^{\prime}}^{2}
$$

Sujeito às restrições: 


$$
\begin{gathered}
-\Delta u_{\max } \leq \Delta u^{a}(k+j \mid k) \leq \Delta u_{\max }, j=0,1, \ldots, m-1 \\
u_{\min } \leq u^{a}(k+j \mid k) \leq u_{\max }, j \in \mathbb{N}
\end{gathered}
$$

Deseja-se que o efeito líquido sobre os modos integradores das ações de controle calculadas em P2b seja idêntico ao de P2a. Em particular, se em P2a foi calculada uma solução em que $x^{i}\left(k+m+\theta_{\max } \mid k\right)=0$, deseja-se que isso também ocorra em P2b. Este feito é obtido através da restrição (3-52), em que $x_{a}^{i}$ (respectivamente $x_{b}^{i}$ ) indica o valor dos modos integradores calculados no problema P2a (respectivamente P2b). Assim, o problema P2b é:

$$
\begin{aligned}
& \min _{\Delta u_{k}^{b}, y_{s p, k}, \delta_{y, k}, \delta_{u, k}} V_{2 b, k}=\sum_{j=0}^{\infty}\left\|y(k+j / k)-y_{s p, k}-\delta_{y, k}\right\|_{Q_{y}}^{2}+\sum_{j=0}^{\infty}\left\|u^{b}(k+j / k)-u_{d e s}-\delta_{u, k}\right\|_{Q_{u}}^{2} \\
& +\sum_{j=0}^{m-1}\left\|\Delta u^{b}(k+j / k)\right\|_{R}^{2}+\left\|\delta_{y, k}\right\|_{S_{y}}^{2}+\left\|\delta_{u, k}\right\|_{S_{u}}^{2}
\end{aligned}
$$

Sujeito às restrições:

$$
\begin{gathered}
-\Delta u_{\max } \leq \Delta u^{b}(k+j \mid k) \leq \Delta u_{\max }, j=0,1, \ldots, m-1 \\
u_{\min } \leq u^{b}(k+j \mid k) \leq u_{\max }, j \in \mathbb{N} \\
u^{b}(k+m-1 \mid k)-u_{d e s}-\delta_{u, k}=0 \\
y_{\min } \leq y_{s p, k} \leq y_{\max } \\
x^{s}\left(k+m+\theta_{\max } \mid k\right)-y_{s p, k}-\delta_{y, k}=0 \\
x_{a}^{i}\left(k+m+\theta_{\max } \mid k\right)=x_{b}^{i}\left(k+m+\theta_{\max } \mid k\right)
\end{gathered}
$$




\subsection{REPRESENTAÇÃO DOS PROBLEMAS DE OTIMIZAÇÃO COMO PROGRAMAÇAO QUADRÁTICA}

Todos os problemas de otimização descritos anteriormente podem ser caracterizados como programação quadrática $(\mathrm{QP})$ pois as restrições são lineares e a função objetivo é quadrática. A forma geral desse tipo de problema é:

$$
\begin{gathered}
\min _{x} V=x^{T} H x+2 c f^{T} x+c \\
\text { sujeito a } \\
\qquad \begin{array}{c}
A_{1} x=0 \\
A_{2} x \leq 0
\end{array}
\end{gathered}
$$

A vantagem de utilizar programação quadrática é a maior robustez dos algoritmos comerciais e a garantia de que todos os pontos de ótimo são globais, pois o problema é convexo. Algumas manipulações algébricas devem ser feitas com as restrições e funções objetivos para colocar os problemas na forma (3-53). As próximas seções indicam com todos os detalhes os procedimentos realizados para o controlador em dois passos.

Os desenvolvimentos expostos são apenas os relevantes ao caso sem distúrbios medidos e utilizando variáveis complexas. Como para o problema em um passo a metodologia é a mesma, neste caso apresenta-se apenas a forma final da função objetivo e das restrições

\subsubsection{Transformando P2a em um problema de programação quadrática}

O problema de otimização P2a é definido pela função-objetivo indicada em (3-43) e pelas restrições (3-44)-(3-45).

Para escrever a função-objetivo de P2a em termos das variáveis de decisão, primeiramente é necessário relacionar as predições dos estados futuros com as ações de controle. De acordo com o modelo do sistema (3-6): 


$$
\begin{aligned}
x(k+1 \mid k) & =A x(k)+B \Delta u(k \mid k) \\
x(k+2 \mid k) & =A x(k+1 \mid k)+B \Delta u(k+1 \mid k) \\
& =A^{2} x(k)+A B \Delta u(k \mid k)+B \Delta u(k+1 \mid k) \\
& \vdots \\
x(k+m \mid k) & =A^{m} x(k)+A^{m-1} B \Delta u(k \mid k)+A^{m-2} B \Delta u(k+1 \mid k)+\ldots B \Delta u(k+m-1 \mid k)
\end{aligned}
$$

Seja $C o$ a matriz definida por

$$
C o=\left[\begin{array}{lllll}
A^{m-1} B & A^{m-2} B & \ldots & A B & B
\end{array}\right]
$$

Assim, o estado predito após $m$ passos pode ser escrito na seguinte forma compacta:

$$
x(k+m \mid k)=A^{m} x(k)+\operatorname{Co\Delta } u_{k}
$$

A forma de calcular a predição após o instante $m$ deve ser ligeiramente modificada pois $\Delta u(k+j \mid k)=0, j \geq m$. Assim, a evolução do estado passa a depender somente da matriz A:

$$
\begin{aligned}
x(k+m+1 \mid k) & =A x(k+m \mid k) \\
x(k+m+2 \mid k) & =A x(k+m+1 \mid k) \\
& =A^{2} x(k+m \mid k) \\
& \vdots \\
x\left(k+m+\theta_{\max }\right) & =A^{\theta_{\max }} x(k+m \mid k) \\
& =A^{\theta_{\max }}\left(A^{m} x(k)+C o \Delta u_{k}\right)
\end{aligned}
$$

Seja $N_{i}$ a matriz definida por

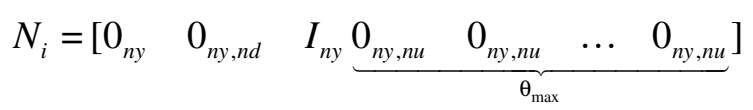

Por construção, $N_{i} x(k)=x^{i}(k)$ e portanto $N_{i} x\left(k+m+\theta_{\text {max }} \mid k\right)=x^{i}\left(k+m+\theta_{\text {max }} \mid k\right)$. Dessa forma, é possível expressar a função-objetivo em função de $\Delta u_{k}^{a}$ : 


$$
\begin{aligned}
\min _{\Delta u_{k}^{a}} V_{2 a, k} & =\left\|x^{i}\left(k+m+\theta_{\max } \mid k\right)\right\|_{Q_{i}}^{2}+\left\|\Delta u_{k}^{a}\right\|_{\bar{R}^{\prime}}^{2} \\
& =\left\|N_{i} A^{\theta_{\max }}\left(A^{m} x(k)+\operatorname{Co} \Delta u_{k}^{a}\right)\right\|_{Q_{i}}^{2}+\left\|\Delta u_{k}^{a}\right\|_{\bar{R}^{\prime}}^{2}
\end{aligned}
$$

As restrições de P2a são limites nas ações de controle e nos valores das entradas do sistema. Reescrevendo essas inequações:

$$
\begin{gathered}
-\Delta u_{\max } \leq \Delta u^{a}(k+j \mid k) \leq \Delta u_{\max }, j=0,1, \ldots, m-1 \\
u_{\min } \leq u^{a}(k+j \mid k) \leq u_{\max }, j \in \mathbb{N}
\end{gathered}
$$

As desigualdades contidas em (3-44) podem ser escritas em termos de $\Delta u_{k}^{a}$ de forma bastante direta:

$$
\underbrace{\left[\begin{array}{c}
-\Delta u_{\max } \\
\vdots \\
-\Delta u_{\max }
\end{array}\right]}_{-\Delta U_{\max }} \leq \Delta u_{k}^{a} \leq \underbrace{\left[\begin{array}{c}
\Delta u_{\max } \\
\vdots \\
\Delta u_{\max }
\end{array}\right]}_{\Delta U_{\max }}
$$

Já para deixar (3-45) em função de $\Delta u_{k}^{a}$, é preciso relacionar primeiramente o vetor de ações de controle com as entradas calculadas:

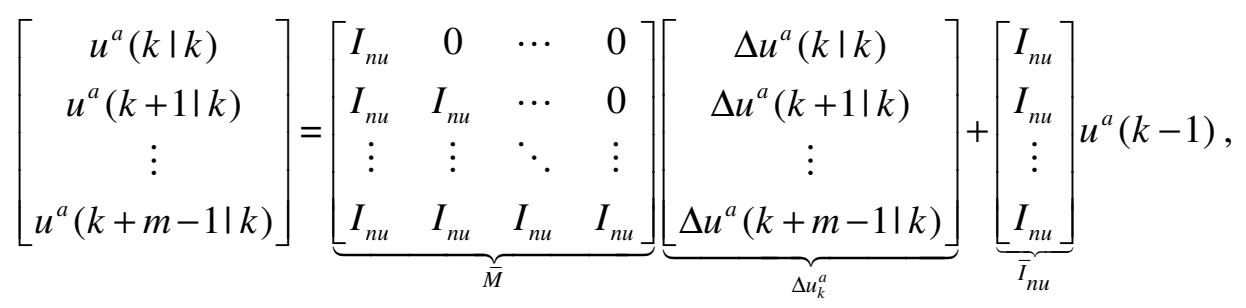

Logo, os limites podem ser impostos através da restrição: 


$$
\underbrace{\left[\begin{array}{c}
I_{n u} \\
I_{n u} \\
\vdots \\
I_{n u}
\end{array}\right]}_{I_{n u}} u_{\min } \leq \bar{M} \Delta u_{k}^{a}+\bar{I}_{n u} u^{a}(k-1) \leq \underbrace{\left[\begin{array}{c}
I_{n u} \\
I_{n u} \\
\vdots \\
I_{n u}
\end{array}\right]}_{I_{n u}} u_{\max }
$$

Reescrevendo na forma $A x \leq b$,

$$
\left[\begin{array}{c}
\bar{M} \\
-\bar{M}
\end{array}\right] \Delta u_{k}^{a} \leq\left[\begin{array}{l}
\bar{I}_{n u}\left(u_{\max }-u^{a}(k-1)\right) \\
\bar{I}_{n u}\left(u(k-1)-u_{\min }\right)
\end{array}\right]
$$

Portanto, o problema P2a pode ser implementado utilizando uma rotina de programação quadrática ao considerar (3-59) como função-objetivo, (3-60) e (3-63) como restrições de desigualdade.

\subsubsection{Transformando P2b num problema de programação quadrática}

Procedimento análogo deve ser feito para o problema P2b, definido originalmente pela função-objetivo indicada em (3-46) e pelas restrições (3-47) a (3-52). Primeiramente, pode-se explicitar a forma quadrática da função-objetivo através de algumas manipulações elementares. Escrevendo os somatórios de forma ligeiramente diferente de (3-46), tem-se: 


$$
\begin{aligned}
V_{2 b, k}= & \underbrace{\sum_{j=0}^{m+\theta_{\max }}\left\|y(k+j \mid k)-y_{s p, k}-\delta_{y, k}\right\|_{Q_{y}}^{2}}_{T_{1}} \\
& +\underbrace{\sum_{j=m+\theta_{\max }+1}^{\infty}\left\|y(k+j \mid k)-y_{s p, k}-\delta_{y, k}\right\|_{Q_{y}}^{2}}_{T_{2}} \\
& +\underbrace{\sum_{j=0}^{m-1}\left\|u^{b}(k+j \mid k)-u_{d e s}-\delta_{u, k}\right\|_{Q_{u}}^{2}}_{T_{3}} \\
& +\underbrace{\sum_{j=m}^{\infty}\left\|u^{b}(k+j \mid k)-u_{d e s}-\delta_{u, k}\right\|_{Q_{u}}^{2}}_{T_{4}} \\
& +\underbrace{\sum_{j=0}^{m-1}\left\|\Delta u^{b}(k+j \mid k)\right\|_{R}^{2}}_{T_{5}} \\
& +\left\|\delta_{u, k}\right\|_{S_{u}}^{2}+\left\|\delta_{y, k}\right\|_{S_{y}}^{2}
\end{aligned}
$$

Para escrever o termo $T_{1}$ num formato apropriado, nota-se que a relação entre as ações de controle e saídas previstas é similar a (3-57), em que as ações de controle eram relacionadas aos estados previstos. Portanto, as predições de saídas entre o instante atual e os próximos $m+\theta_{\max }$ períodos de amostragem são:

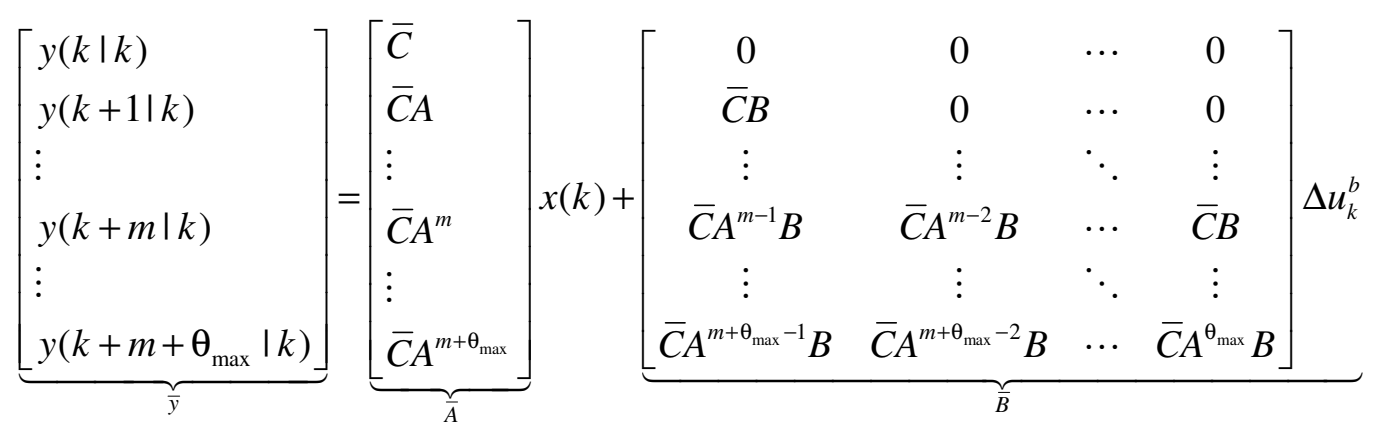

Na equação anterior, $\bar{C}$ corresponde à parte de $\mathrm{C}$ relativa apenas as saídas físicas de fato, ou

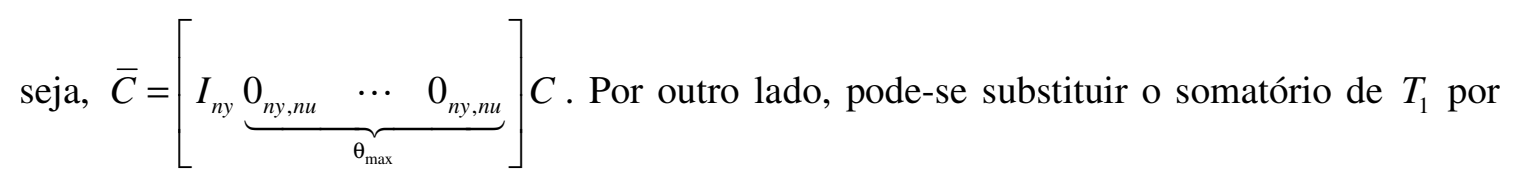
um produto escalar conveniente: 


$$
\begin{aligned}
T_{1} & =\sum_{j=0}^{m+\theta_{\max }}\left\|y(k+j \mid k)-y_{s p, k}-\delta_{y, k}\right\|_{Q_{y}}^{2} \\
& =\left\|\bar{y}(k \mid k)-\bar{I}_{n y} y_{s p, k}-\bar{I}_{n y} \delta_{y, k}\right\|_{\bar{Q}_{y}}^{2}
\end{aligned}
$$

sendo

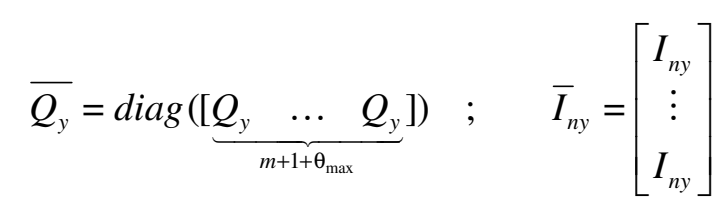

Usando a relação (3-65) em (3-66), chega-se à seguinte expressão para $T_{1}$ :

$$
T_{1}=\left\|\bar{A} x(k)+\bar{B} \Delta u_{k}^{b}-\bar{I}_{n y} y_{s p, k}-\bar{I}_{n y} \delta_{y, k}\right\|_{\bar{Q}_{y}}^{2}
$$

Para o termo $T_{2}$, pode-se inicialmente relacionar as predições de saída em função dos estados previstos usando a definição do modelo feita em (3-8), ou seja:

$$
y(k)=x^{s}(k)+\Psi x^{d}(k)
$$

Portanto,

$$
\begin{aligned}
T_{2} & =\sum_{j=m+\theta_{\max }+1}^{\infty}\left\|y(k+j \mid k)-y_{s p, k}-\delta_{y, k}\right\|_{Q_{y}}^{2} \\
& =\sum_{j=1}^{\infty}\left\|x^{s}\left(k+j+m+\theta_{\max } \mid k\right)+\Psi x^{d}\left(k+j+m+\theta_{\max } \mid k\right)-y_{s p, k}-\delta_{y, k}\right\|_{Q_{y}}^{2}
\end{aligned}
$$

Lembrando que a restrição (3-51) impõe que $x^{s}\left(k+m+\theta_{\max } \mid k\right)-y_{s p, k}-\delta_{y, k}=0$; tem-se simplesmente: 


$$
\begin{aligned}
T_{2} & =\sum_{j=1}^{\infty}\left\|\Psi x^{d}\left(k+j+m+\theta_{\max } \mid k\right)\right\|_{Q_{y}}^{2} \\
& =\sum_{j=1}^{\infty}\left\|\Psi F^{j} x^{d}\left(k+m+\theta_{\max } \mid k\right)\right\|_{Q_{y}}^{2} \\
& =x^{d}\left(k+m+\theta_{\max } \mid k\right)^{T}(\underbrace{\sum_{j=1}^{\infty} F^{j^{T}} \Psi^{T} Q_{y} \Psi F^{j}}_{\bar{Q}}) x^{d}\left(k+m+\theta_{\max } \mid k\right)
\end{aligned}
$$

A série infinita que define $\bar{Q}$ pode ser calculada ao resolver a seguinte equação de Lyapunov,

$$
\bar{Q}=F^{T} \Psi^{T} Q_{y} \Psi F+F^{T} \bar{Q} F
$$

Definindo $N_{d}$ de forma análoga a $N_{i}$, ou seja,

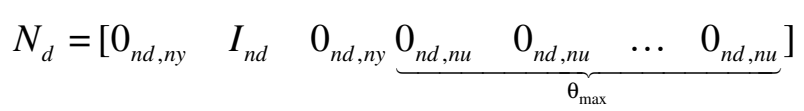

Assim, as predições dos estados $x^{d}$ podem ser calculadas usando-se (3-57):

$$
x^{d}\left(k+m+\theta_{\max } \mid k\right)=N_{d} A^{\theta_{\max }}\left[A^{m} x(k \mid k)+C o \Delta u_{k}^{b}\right]
$$

Logo, a expressão de $T_{2}$ em termos das variáveis de decisão é:

$$
T_{2}=\left\|N_{d} A^{\theta_{\max }}\left[A^{m} x(k)+\operatorname{Co\Delta } u_{k}^{b}\right]\right\|_{\bar{Q}}^{2}
$$

O termo $T_{3}$ pode ser escrito de forma sintética utilizando-se a relação entre as ações de controle e as entradas da planta calculada em (3-61):

$$
\begin{aligned}
T_{3} & =\sum_{j=0}^{m-1}\left\|u(k+j \mid k)-u_{d e s}-\delta_{u, k}\right\|_{\varrho_{u}}^{2} \\
& =\left\|\bar{M} \Delta u_{k}^{b}+\bar{I}_{n u} u(k-1)-\bar{I}_{n u} u_{d e s}-\bar{I}_{n u} \delta_{u, k}\right\|_{\bar{Q}_{u}}^{2}
\end{aligned}
$$


sendo $\overline{Q_{u}}=\operatorname{diag}(\underbrace{Q_{u} \quad \ldots \quad Q_{u}}_{m})$.

Já o termo $T_{4}$ é igual a 0 de acordo com a restrição (3-49), ou seja, como $\Delta u(k+j \mid k)=0$ para $j \geq m$, se

$$
u^{b}(k+m-1 \mid k)-u_{d e s}-\delta_{u, k}=0
$$

então

$$
u^{b}(k+m+j-1 \mid k)-u_{\text {des }}-\delta_{u, k}=0, j \geq 0
$$

Logo, todas as parcelas de $T_{4}$ são de fato nulas.

Finalmente, da definição de $\Delta u_{k}^{b}$ tem-se que

$$
T_{5}=\sum_{j=0}^{m-1}\|\Delta u(k+j \mid k)\|_{R}^{2}=\left\|\Delta u_{k}^{b}\right\|_{\bar{R}}^{2}
$$

sendo $\bar{R}=\operatorname{diag}(\underbrace{R \quad \ldots \quad R}_{m})$.

Escrevendo todos os termos, a função-objetivo é dada por:

$$
\begin{aligned}
V_{2 b, k}= & \left\|\bar{A} x(k \mid k)+\bar{B} \Delta u_{k}^{b}-\bar{I}_{n y} y_{s p, k}-\bar{I}_{n y} \delta_{y, k}\right\|_{\bar{Q}_{y}}^{2} \\
& +\left\|N_{d} A^{\theta_{\max }}\left[A^{m} x(k)+C o \Delta u_{k}^{b}\right]\right\|_{\bar{Q}}^{2} \\
& +\left\|\bar{M} \Delta u_{k}^{b}+\bar{I}_{n u} u(k-1)-\bar{I}_{n u} u_{d e s}-\bar{I}_{n u} \delta_{u, k}\right\|_{\bar{Q}_{u}}^{2} \\
& +\left\|\Delta u_{k}^{b}\right\|_{\bar{R}}^{2} \\
& +\left\|\delta_{u, k}\right\|_{S_{u}}^{2}+\left\|\delta_{y, k}\right\|_{s_{y}}^{2}
\end{aligned}
$$




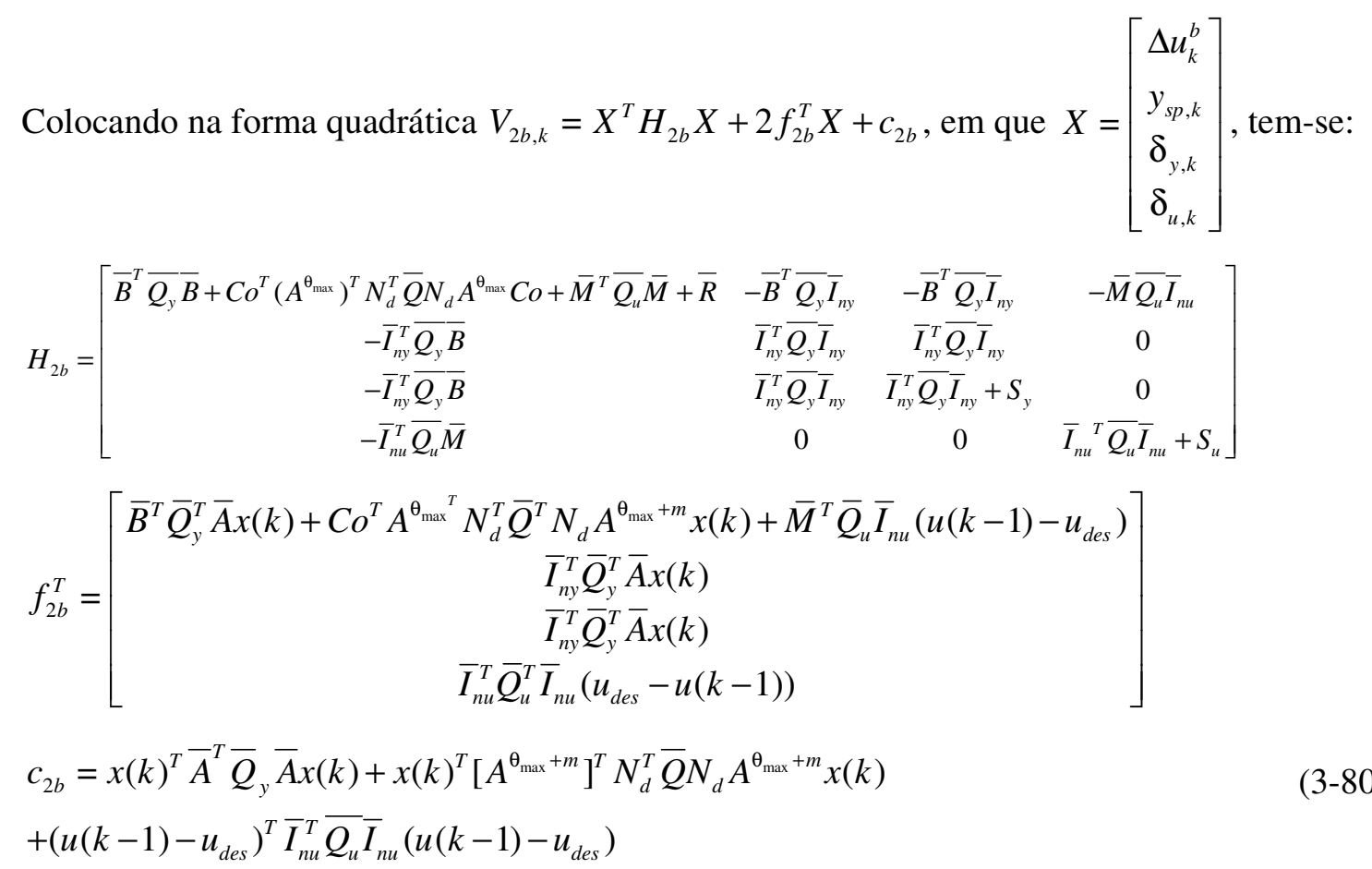

As restrições do problema já foram definidas anteriormente. Reescrevendo-as para tornar mais clara a situação, tem-se:

$$
\begin{gathered}
-\Delta u_{\max } \leq \Delta u^{b}(k+j \mid k) \leq \Delta u_{\max }, j=0,1, \ldots, m-1 \\
u_{\min } \leq u^{b}(k+j \mid k) \leq u_{\max }, j \in \mathbb{N} \\
u^{b}(k+m-1 \mid k)-u_{d e s}-\delta_{u, k}=0 \\
y_{\min } \leq y_{s p, k} \leq y_{\max } \\
x^{s}\left(k+m+\theta_{\max } \mid k\right)-y_{s p, k}-\delta_{y, k}=0 \\
x_{a}^{i}\left(k+m+\theta_{\max } \mid k\right)=x_{b}^{i}\left(k+m+\theta_{\max } \mid k\right)
\end{gathered}
$$

É preciso expressar cada uma delas em função das variáveis de decisão. As restrições de desigualdade desse problema advêm de duas fontes: valores mínimos e máximos para as entradas do sistema e limites sobre as variáveis de decisão $\Delta u_{k}^{b}$ e $y_{s p, k}$.

Para representar o primeiro tipo (restrição (3-48)), procede-se de maneira similar ao realizado com a restrição (3-45) do problema $\mathrm{P} 2 \mathrm{a}$ : 


$$
\begin{gathered}
u_{\min } \leq u^{b}(k+j \mid k) \leq u_{\max }, j \in \mathbb{N} \\
\bar{I}_{n u} u_{\min } \leq \bar{M} \Delta u_{k}^{b}+\bar{I}_{n u} u(k-1) \leq \bar{I}_{n u} u_{\max } \\
{\left[\begin{array}{cccc}
\bar{M} & 0 & 0 & 0 \\
-\bar{M} & 0 & 0 & 0
\end{array}\right]\left[\begin{array}{c}
\Delta u_{k}^{b} \\
y_{s p, k} \\
\delta_{y, k} \\
\delta_{u, k}
\end{array}\right] \leq\left[\begin{array}{l}
\bar{I}_{n u}\left(u_{\max }-u(k-1)\right) \\
\bar{I}_{n u}\left(u(k-1)-u_{\min }\right)
\end{array}\right]}
\end{gathered}
$$

Por outro lado, os limites sobre as variáveis são dados diretamente por:

$$
\left[\begin{array}{c}
-\Delta u_{\max } \\
y_{\min } \\
-\infty \\
-\infty
\end{array}\right] \leq\left[\begin{array}{c}
\Delta u_{k}^{b} \\
y_{s p, k} \\
\delta_{y, k} \\
\delta_{u, k}
\end{array}\right] \leq\left[\begin{array}{c}
\Delta u_{\max } \\
y_{\max } \\
+\infty \\
+\infty
\end{array}\right]
$$

Procedendo agora à análise das restrições de igualdade, considere-se a relação entre estados previstos e entradas calculadas deduzida anteriormente,

$$
x\left(k+m+\theta_{\max }\right)=A^{\theta_{\max }}\left(A^{m} x(k)+C o \Delta u_{k}^{b}\right)
$$

As relações que envolvem as componentes $x^{s}$, são obtidas de forma análoga ao feito com $x^{i}$ no problema P2a. Seja $N_{s}$ dada por:

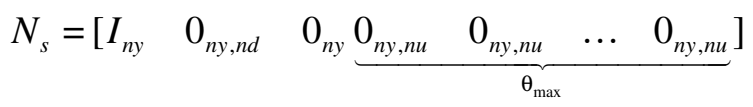

Assim, a restrição (3-51) é equivalente a

$$
N_{s}\left(A^{\theta_{\max }+m} x(k)+A^{\theta_{\max }} \operatorname{Co\Delta } \Delta u_{k}^{b}\right)-I_{n y} y_{s p, k}-I_{n y} \delta_{y, k}=0
$$

Da mesma forma, (3-52) é equivalente a: 


$$
\begin{aligned}
& x_{a}^{i}\left(k+m+\theta_{\max } \mid k\right)=x_{b}^{i}\left(k+m+\theta_{\max } \mid k\right) \\
& N_{i} A^{\theta_{\max }}\left(A^{m} x(k)+C o \Delta u_{k}^{a}\right)=N_{i} A^{\theta_{\max }}\left(A^{m} x(k)+C o \Delta u_{k}^{b}\right) \\
& N_{i} A^{\theta_{\max }} C o \Delta u_{k}^{a^{*}}=N_{i} A^{\theta_{\max }} \operatorname{Co\Delta } u_{k}^{b}
\end{aligned}
$$

Note que $\Delta u_{k}^{a^{*}}$ é o valor ótimo de $\Delta u_{k}^{a}$ obtido na solução do problema P2a correspondente ao instante $\mathrm{k}$, de maneira que a única variável de decisão presente em (3-85) é $\Delta u_{k}^{b}$.

Por fim, a restrição (3-49) pode ser expressa em função de $\Delta u_{k}^{b}$ da seguinte forma:

$$
\begin{aligned}
& u^{b}(k+m-1 \mid k)-u_{d e s}-\delta_{u, k}=0 \\
& u(k-1)+\sum_{j=0}^{m-1} \Delta u^{b}(k+j \mid k)-u_{d e s}-\delta_{u, k}=0 \\
& u(k-1)+\underbrace{\left[\begin{array}{llll}
I_{n u} & I_{n u} & \ldots & I_{n u}
\end{array}\right]}_{\bar{I}_{n u}^{T}}\left[\begin{array}{c}
\Delta u^{b}(k \mid k) \\
\Delta u^{b}(k+1 \mid k) \\
\vdots \\
\Delta u^{b}(k+m-1 \mid k)
\end{array}\right]-u_{d e s}-\delta_{u, k}=0 \\
& \bar{I}_{n u}^{T} \Delta u_{k}^{b}-I_{n u} \delta_{u, k}=u_{d e s}-u(k-1)
\end{aligned}
$$

Finalmente, as restrições de igualdade podem ser agrupadas numa única equação matricial:

$$
\left[\begin{array}{cccc}
N_{i} A^{\theta_{\max }} C o & 0 & 0 & 0 \\
N_{s} A^{\theta_{\max }} C o & -I_{n y} & -I_{n y} & 0 \\
\bar{I}_{n u}^{T} & 0 & 0 & -I_{n u}
\end{array}\right]\left[\begin{array}{c}
\Delta u_{k}^{b} \\
y_{s p, k} \\
\delta_{y, k} \\
\delta_{u, k}
\end{array}\right]=\left[\begin{array}{c}
N_{i} A^{\theta_{\max }} C o \Delta u_{k}^{a^{*}} \\
-N_{s} A^{\theta_{\max }+m} x(k) \\
u_{d e s}-u(k-1)
\end{array}\right]
$$

Por fim, considera-se que a segunda restrição em (3-87) deve ser escrita apenas para as saídas que se deseja manter na faixa, enquanto a última restrição deve ser implementada apenas para as entradas com target. 


\subsubsection{Transformando P1 em um problema de programação quadrática}

Colocando (3-41) na forma quadrática $V_{1}=X^{T} H_{1} X+2 c f_{1}^{T} X+c 1$, em que $X=\left[\begin{array}{c}\Delta u_{k} \\ y_{s p, k} \\ \delta_{y, k} \\ \delta_{u, k} \\ \delta_{i, k}\end{array}\right]$, tem-se:

$H_{1}=\left[\begin{array}{ccccc}\bar{B}^{T} \overline{Q_{y}} \bar{B}+C O^{T}\left(A^{\theta_{\max }}\right)^{T} N_{d}^{T} \overline{Q^{\prime}} N_{d} A^{\theta_{\max }} C o+\bar{M}^{T} \overline{Q_{u}} \bar{M}+\bar{R} & -\bar{B}^{T} \bar{Q}_{y} \bar{I}_{n y} & -\bar{B}^{T} \bar{Q}_{y} \bar{I}_{n y} & -\bar{M} \bar{Q}_{u} \bar{I}_{n u} & 0 \\ -\bar{I}_{n y}^{T} \overline{Q_{y}} \bar{B} & \bar{I}_{n y}^{T} \overline{Q_{y}} \bar{I}_{n y} & \bar{I}^{T}{ }^{T} \bar{Q}_{y} \bar{I}_{n y} & 0 & 0 \\ -\bar{I}_{n y}^{T} \overline{Q_{y}} \bar{B} & \bar{I}_{n y}^{T} \overline{Q_{y}} \bar{I}_{n y} & \bar{I}_{n y}^{T} \overline{Q_{y}} \bar{I}_{n y}+S_{y} & 0 & 0 \\ -\bar{I}_{n u}^{T} \overline{Q_{u}} \bar{M} & 0 & 0 & \bar{I}_{n u}{ }^{T} \overline{Q_{u}} \bar{I}_{n u}+S_{u} & 0 \\ 0 & 0 & 0 & 0 & S_{i}\end{array}\right]$

$f_{1}^{T}=\left[\begin{array}{c}x(k)^{T} \bar{A}^{T} \bar{Q}_{y} \bar{B}+x(k)^{T}\left[A^{\theta_{\max }+m}\right]^{T} N_{d}^{T} \bar{Q} N d A^{\theta_{\max }} C o+\left(u(k-1)-u_{d e s}\right)^{T} \bar{I}_{n u}^{T} \overline{Q_{u}} \bar{M} \\ -x(k)^{T} \bar{A}^{T} \bar{Q}_{y} \bar{I}_{n y} \\ -x(k)^{T} \bar{A}^{T} \bar{Q}_{y} \bar{I}_{n y} \\ \left(u_{d e s}-u(k-1)\right)^{T} \bar{I}_{n u}^{T} \bar{Q}_{u} \bar{I}_{n u} \\ 0\end{array}\right]$

$$
\begin{aligned}
c_{1}= & x(k)^{T} \bar{A}^{T} \bar{Q}_{y} \bar{A} x(k)+x(k)^{T}\left[A^{\theta_{\max }+m}\right]^{T} N_{d}^{T} \bar{Q} N_{d} A^{\theta_{\max }+m} x(k) \\
& +\left(u(k-1)-u_{d e s}\right)^{T} \bar{I}_{n u}^{T} \overline{Q_{u}} \bar{I}_{n u}\left(u(k-1)-u_{\text {des }}\right)
\end{aligned}
$$
se:

$$
\left[\begin{array}{ccccc}
\bar{M} & 0 & 0 & 0 & 0 \\
-\bar{M} & 0 & 0 & 0 & 0
\end{array}\right]\left[\begin{array}{c}
\Delta u_{k} \\
y_{s p, k} \\
\delta_{y, k} \\
\delta_{u, k} \\
\delta_{i, k}
\end{array}\right] \leq\left[\begin{array}{l}
\bar{I}_{n u}^{T}\left(u_{\max }-u(k-1)\right) \\
\bar{I}_{n u}^{T}\left(u(k-1)-u_{\min }\right)
\end{array}\right]
$$
matricial:

Finalmente, as restrições de igualdade podem ser agrupadas numa única equação 


$$
\left[\begin{array}{ccccc}
N_{i} A^{\theta_{\max }} C o & 0 & 0 & 0 & -I_{n y} \\
N_{s} A^{\theta_{\max }} C o & -I_{n y} & -I_{n y} & 0 & 0 \\
\bar{I}_{n u} & 0 & 0 & -I_{n u} & 0
\end{array}\right]\left[\begin{array}{c}
\Delta u_{k} \\
y_{s p, k} \\
\delta_{y, k} \\
\delta_{u, k} \\
\delta_{i, k}
\end{array}\right]=\left[\begin{array}{c}
N_{i} A^{\theta_{\max }+m} x(k) \\
-N_{s} A^{\theta_{\max }+m} x(k) \\
u_{d e s}-u(k-1)
\end{array}\right]
$$

Por fim, os limites sobre as variáveis são dados por:

$$
\left[\begin{array}{c}
-\Delta u_{k \max } \\
y_{\min } \\
-\infty \\
-\infty \\
-\infty
\end{array}\right] \leq\left[\begin{array}{c}
\Delta u_{k} \\
y_{s p, k} \\
\delta_{y, k} \\
\delta_{u, k} \\
\delta_{i, k}
\end{array}\right] \leq\left[\begin{array}{c}
\Delta u_{k \max } \\
y_{\max } \\
+\infty \\
+\infty \\
+\infty
\end{array}\right]
$$




\section{DESENVOLVIMENTO DE UM OBSERVADOR HEURÍSTICO}

O controlador proposto neste trabalho utiliza o estado $x$ para suas predições. Este estado deve ser estimado a partir da dinâmica da planta. Como o modelo utilizado pelo controlador é sempre diferente do comportamento real do sistema, sabe-se que essa estimativa será intrinsecamente inexata.

Ao se sintetizar um observador, uma das providências é garantir que a diferença entre o estado estimado e o estado real convirja assintoticamente para 0 . No entanto, outros requisitos podem ser adicionados a esse problema. Por exemplo, é possível buscar um desempenho ótimo diante de um padrão esperado para os ruídos do processo e de medida, levando ao Filtro de Kalman. Essa estratégia está descrita na seção 4.1.

Outra possibilidade, não abordada no escopo desta dissertação, seria a implementação de um filtro robusto a erros de modelagem. Dessa forma seria possível ter garantias mínimas da performance do sistema em função da incerteza esperada. Neste trabalho analisou-se uma formulação heurística de um observador, sem garantias de otimalidade, mas com uma dinâmica inicial mais rápida. Em outros termos, o erro da estimação nos primeiros instantes de estimação tende a ser menor, permitindo que o controlador tome decisões mais acertadas quando o desvio entre as condições real e desejada da planta for grande. Este estimador é uma extensão da técnica proposta em Rodrigues (2001). A descrição completa de como esse observador é obtido está exposta na seção 4.2.

\subsection{FILTRO DE KALMAN}

O modelo utilizado pelo controlador é da forma

$$
\begin{aligned}
& x(k+1)=A \cdot x(k)+B \cdot \Delta u(k) \\
& y(k)=C \cdot x(k) \\
& x \in \mathbb{R}^{n x}, \Delta u \in \mathbb{R}^{n u}, y \in \mathbb{R}^{n y}
\end{aligned}
$$

O filtro de Kalman deverá estimar todo o estado $x$, utilizando-se uma relação de retroalimentação do erro de estimação: 


$$
\begin{aligned}
& \hat{x}(k+1 \mid k)=A \hat{x}(k \mid k)+B \Delta u(k \mid k) \\
& \hat{x}(k+1 \mid k+1)=\hat{x}(k+1 \mid k)+K_{K}(y(k+1)-C \hat{x}(k+1 \mid k))
\end{aligned}
$$

Como visto no capítulo para o cálculo do ganho do filtro, $K_{K}$, é necessário conhecer as matrizes de covariância $R_{1}, R_{2}$ e $R_{12}$, definidas na seção 2.3. Em todas as simulações realizadas, supôs-se que os ruídos não eram correlacionados, ou seja, fez-se $R_{12}=0$.

Por outro lado, para representar a situação prática em que a covariância do ruído real na planta é desconhecida, fixaram-se $R_{1}$ e $R_{2}$ como matrizes identidade $\left(R_{1}=I_{n x}, R_{2}=I_{n y}\right)$. Também a matriz $N$, que associa o ruído de processo ao estado, foi considerada igual a $I_{n x}$. As expressões utilizadas no cálculo do filtro foram, portanto:

$$
\begin{gathered}
P=A P A^{T}+I_{n x}-\left(A P C^{T}\right)\left(C P C^{T}+I_{n y}\right)^{-1}\left(C P A^{T}\right) \\
K=\left(A P C^{T}\right)\left(C P C^{T}+I_{n y}\right)^{-1}
\end{gathered}
$$

\subsection{OBSERVADOR HEURÍSTICO}

Consideremos um sistema linear cuja dinâmica é descrita pelas equações (4-1). O observador proposto é baseado na realocação dos pólos de um sistema fictício associado ao sistema definido em (4-1):

$$
x_{F}(k+1)=\left(I-K_{F} C\right) A \cdot x_{F}(k)
$$

em que $I$ é a matriz identidade de dimensão $n x$ e $K_{F} \in \mathbb{R}^{n x \times n y}$ é uma matriz a ser determinada. Para que o erro de estimação convirja a zero, é necessário que os autovalores da matriz $\left(I-K_{F} C\right) A$ estejam contidos no interior do disco de raio unitário. Isso é equivalente à condição de estabilidade via equação de Lyapunov, ou seja, à existência de uma matriz $P_{F}=P_{F}^{T}>0$ tal que

$$
\left[\left(I-K_{F} C\right) A\right]^{T} P_{F}\left[\left(I-K_{F} C\right) A\right]-P_{F}<0
$$


Com o intuito de transformar o problema de encontrar $K_{F}, P_{F}$ através de técnicas de LMI, é conveniente introduzir uma variável $\lambda \in \mathbb{R}$ de modo que:

$$
\left[\left(I-K_{F} C\right) A\right]^{T} P_{F}\left[\left(I-K_{F} C\right) A\right]-P_{F}<\lambda I
$$

Portanto, o sistema será estável se for possível encontrar $K_{F}, P_{F}, \lambda$ que satisfaçam (4-6) e com $\lambda<0$. O procedimento para encontrar um observador pode ser escrito em termos de um problema otimização da seguinte forma:

$$
\begin{aligned}
& \min _{K_{F}, P_{F}, \lambda} \lambda \\
& \text { sujeito a } \\
& \lambda I+P_{F}-\left[\left(I-K_{F} C\right) A\right]^{T} P_{F}\left[\left(I-K_{F} C\right) A\right]>0
\end{aligned}
$$

Utilizando o Complemento de Schur, pode-se reescrever a restrição de (4-7) na forma:

$$
\left[\begin{array}{cc}
P_{F} & P_{F}\left(I-K_{F} C\right) A \\
\left(\left(I-K_{F} C\right) A\right)^{T} P_{F} & P_{F}+\lambda I
\end{array}\right]>0
$$

No entanto, a expressão resultante não é linear em relação às variáveis pois há multiplicações entre $P_{F}$ e $K_{F}$. Esta formulação foi originalmente proposta em Rodrigues (2001). Tem-se uma BMI, um problema não convexo e de tratamento numérico mais instável. Neste trabalho, é proposta uma técnica alternativa para a resolução dessa BMI.

Fixando-se $P_{F}$ ou $K_{F}$, tem-se uma LMI na outra variável. Portanto, um possível algoritmo heurístico consiste em resolver alternadamente problemas com $P_{F}$ ou $K_{F}$ mantidas constantes. O algoritmo detalhado é dado a seguir:

1. Uma estimativa inicial para $K_{F}$ é obtida através da equação que define o Filtro de Kalman estacionário. Seja $K_{F}^{i}$ essa estimativa.. Continua-se no passo 2. 
2. Resolve-se o problema

$$
\begin{aligned}
& \min _{P_{F}, \lambda} \lambda \\
& \text { sujeito a } \\
& {\left[\begin{array}{cc}
P_{F} & P_{F}\left(I-K_{F}^{i} C\right) A \\
\left(\left(I-K_{F}^{i} C\right) A\right)^{T} P_{F} & P_{F}+\lambda I
\end{array}\right]>0} \\
& P_{F}=P_{F}^{T}>0 \\
& P_{F} P_{F}^{T}<\alpha I
\end{aligned}
$$

O valor de $\alpha \in \mathbb{R}$ é escolhido arbitrariamente para limitar os autovalores de $P_{F}$, de modo a melhorar o condicionamento numérico do problema.

Define-se $P_{F}^{i}=P_{F}$. Continua-se no passo 3.

3. Resolve-se o problema

$$
\begin{aligned}
& \min _{K_{F}, \lambda} \lambda \\
& \text { sujeito a } \\
& {\left[\begin{array}{cc}
P_{F}^{i} & P_{F}^{i}\left(I-K_{F} C\right) A \\
\left(\left(I-K_{F} C\right) A\right)^{T} P_{F}^{i} & P_{F}^{i}+\lambda I
\end{array}\right]>0}
\end{aligned}
$$

O valor de $K_{F}$ encontrado será utilizado como ganho do observador.

As alterações no procedimento de cálculo desenvolvido por Rodrigues (2001) foram a inclusão da restrição sobre o maior autovalor da matriz $P_{F}$, a inicialização de $K_{F}$ com um valor que garante a viabilidade da restrição (4-6) caso o sistema $(A, C)$ seja detectável. Além disso, o algoritmo proposto naquele trabalho sugere que os passos 2 e 3 definidos anteriormente sejam iterados até que ocorre a convergência para certo par $\left(K_{F}, P_{F}\right)$. Experimentos numéricos sugeriram que durante esse processo de convergência ocorria um aumento na soma dos módulos dos autovalores da matriz $\left(I-K_{F} C\right) A$, levando o observador 
a ter uma dinâmica mais lenta. Assim, substituiu-se o procedimento iterativo pela resolução de (4-9) e (4-10) apenas uma vez.

\subsubsection{Aplicação Ao Modelo Proposto}

A técnica desenvolvida anteriormente pode ser aplicada às representações em espaço de estados propostas na seção 3. O desenvolvimento será detalhado para o modelo da seção 3.2, ou seja, contendo distúrbios medidos.

Para tanto, é conveniente separar o estado $x$ em dois blocos. No primeiro deles, composto pelas componentes $x^{s}, x^{d}, x^{w}, x^{i}$, tem-se a informação da dinâmica de fato do sistema, ou seja, a velocidade de decaimento dos pólos, os modos integradores incrementando a saída, etc. Por outro lado, o segundo bloco é composto por $z_{1}, \cdots, z_{\theta_{\max }} ; z_{1}^{w}, \cdots, z_{\tau_{\max }}^{w}$ e registra apenas um histórico das últimas ações de controle e variações dos distúrbios.

Uma estratégia possível para a síntese do observador é considerar o vetor $X=\left[\begin{array}{llll}x^{s^{T}} & x^{d^{T}} & x^{w^{T}} & x^{i^{T}}\end{array}\right]$ como um estado que realmente precisa ser estimado, enquanto $Z=\left[\begin{array}{llllll}z_{1}^{T} & \cdots & z_{\theta_{\max }}^{T} & z_{1}^{w^{T}} & \cdots & z_{\tau_{\max }^{T}}^{T}\end{array}\right]$ é diretamente mensurável. As dimensões desses estados são, respectivamente, $n X=2 n y+n d+n p$ e $n Z=n u \cdot \theta_{\max }+n w \cdot \tau_{\max }$.

O vetor de saídas também pode ser dividido entre uma componente das saídas de fato do sistema ( $y$ ) e uma componente relativa ao histórico das entradas antigas, dada pelo vetor $Z$ previamente definido.

Com essa divisão, a evolução do sistema pode ser escrita na forma:

$$
\begin{aligned}
& {\left[\begin{array}{c}
X(k+1) \\
Z(k+1)
\end{array}\right]=\left[\begin{array}{cc}
A_{X X} & A_{X Z} \\
0 & A_{Z Z}
\end{array}\right] \cdot\left[\begin{array}{c}
X(k) \\
Z(k)
\end{array}\right]+B \cdot \Delta u(k)+B w \cdot \Delta w(k)} \\
& {\left[\begin{array}{c}
y(k) \\
Z(k)
\end{array}\right]=\left[\begin{array}{cc}
C_{y X} & 0 \\
0 & I_{n Z}
\end{array}\right] \cdot\left[\begin{array}{c}
X(k) \\
Z(k)
\end{array}\right]}
\end{aligned}
$$

Utilizando o símbolo ${ }^{\wedge}$ para indicar valores estimados, a predição de um passo a frente no controlador é dada por 


$$
\begin{aligned}
& {\left[\begin{array}{l}
\hat{X}(k+1 \mid k) \\
\hat{Z}(k+1 \mid k)
\end{array}\right]=\left[\begin{array}{cc}
A_{X X} & A_{X Z} \\
0 & A_{Z Z}
\end{array}\right] \cdot\left[\begin{array}{c}
\hat{X}(k \mid k) \\
\hat{Z}(k \mid k)
\end{array}\right]+B \cdot \Delta u(k)+B w \cdot \Delta w(k)} \\
& {\left[\begin{array}{c}
\hat{y}(k) \\
\hat{Z}(k)
\end{array}\right]=\left[\begin{array}{cc}
C_{y X} & 0 \\
0 & I_{n Z}
\end{array}\right] \cdot\left[\begin{array}{c}
\hat{X}(k \mid k) \\
\hat{Z}(k \mid k)
\end{array}\right]}
\end{aligned}
$$

A notação $\hat{x}(k+1 \mid k)$ indica a estimação do valor que $x$ terá no instante $k+1$, calculada apenas com as informações disponíveis no instante $k$. Essa predição pode ser corrigida com a leitura da evolução real:

$$
\left[\begin{array}{c}
\hat{X}(k+1 \mid k+1) \\
\hat{Z}(k+1 \mid k+1)
\end{array}\right]=\left[\begin{array}{c}
\hat{X}(k+1 \mid k) \\
\hat{Z}(k+1 \mid k)
\end{array}\right]+L\left(\left[\begin{array}{c}
y(k+1) \\
Z(k+1)
\end{array}\right]-\left[\begin{array}{cc}
C_{y X} & 0 \\
0 & I_{n Z}
\end{array}\right] \cdot\left[\begin{array}{c}
\hat{X}(k+1 \mid k) \\
\hat{Z}(k+1 \mid k)
\end{array}\right]\right)
$$

O observador para o sistema completo seria dado por uma matriz $L$ tal que:

$$
L=\left[\begin{array}{cc}
K_{F} & 0 \\
0 & I_{n Z}
\end{array}\right]
$$

Portanto, o procedimento descrito para o cálculo da matriz $K_{F}$ pode ser realizado substituindo as matrizes $A$ e $C$ em (4-1) por $A_{X X}$ e $C_{y X}$, respectivamente.

Para obter maiores informações a respeito da dinâmica desse observador, pode-se substituir (4-12) em (4-13):

$$
\begin{aligned}
& {\left[\begin{array}{c}
\hat{X}(k+1 \mid k+1) \\
\hat{Z}(k+1 \mid k+1)
\end{array}\right]=\left[\begin{array}{cc}
A_{X X} & A_{X Z} \\
0 & A_{Z Z}
\end{array}\right] \cdot\left[\begin{array}{c}
\hat{X}(k \mid k) \\
\hat{Z}(k \mid k)
\end{array}\right]+B \cdot \Delta u(k)+B w \cdot \Delta w(k) } \\
&+ L\left(\left[\begin{array}{c}
y(k+1) \\
Z(k+1)
\end{array}\right]-\left[\begin{array}{cc}
C_{y X} & 0 \\
0 & I_{n Z}
\end{array}\right] \cdot\left[\begin{array}{cc}
A_{X X} & A_{X Z} \\
0 & A_{Z Z}
\end{array}\right] \cdot\left[\begin{array}{c}
\hat{X}(k \mid k) \\
\hat{Z}(k \mid k)
\end{array}\right]+B \cdot \Delta u(k)+B w \cdot \Delta w(k)\right) \\
&=\left(\left[\begin{array}{cc}
I_{n X} & 0 \\
0 & I_{n Z}
\end{array}\right]-L\left[\begin{array}{cc}
C_{y X} & 0 \\
0 & I_{n Z}
\end{array}\right]\right) \cdot\left[\begin{array}{cc}
A_{X X} & A_{X Z} \\
0 & A_{Z Z}
\end{array}\right] \cdot\left[\begin{array}{l}
\hat{X}(k \mid k) \\
\hat{Z}(k \mid k)
\end{array}\right]+ \\
& L\left[\begin{array}{c}
y(k+1) \\
Z(k+1)
\end{array}\right]+\left(I_{n X+n Z}-L C\right)(B \Delta u(k)+B w \cdot \Delta w(k))
\end{aligned}
$$


Usando a forma definida para $L$ em (4-14), tem-se

$$
\begin{aligned}
& {\left[\begin{array}{c}
\hat{X}(k+1 \mid k+1) \\
\hat{Z}(k+1 \mid k+1)
\end{array}\right]=\left[\begin{array}{cc}
I_{n X}-K_{F} C_{y X} & 0 \\
0 & 0
\end{array}\right] \cdot\left[\begin{array}{cc}
A_{X X} & A_{X Z} \\
0 & A_{Z Z}
\end{array}\right] \cdot\left[\begin{array}{c}
\hat{X}(k \mid k) \\
\hat{Z}(k \mid k)
\end{array}\right]} \\
& +\left[\begin{array}{c}
K_{F} \cdot y(k+1) \\
Z(k+1)
\end{array}\right]+\left[\begin{array}{cc}
I_{n X}-K_{F} C_{y X} & 0 \\
0 & 0
\end{array}\right] \cdot\left(\left[\begin{array}{c}
B_{X} \\
B_{Z}
\end{array}\right] \Delta u(k)+\left[\begin{array}{c}
B w_{X} \\
B w_{Z}
\end{array}\right] \Delta w(k)\right) \\
& {\left[\begin{array}{c}
\hat{X}(k+1 \mid k+1) \\
\hat{Z}(k+1 \mid k+1)
\end{array}\right]=\left[\begin{array}{cc}
\left(I_{n X}-K_{F} C_{y X}\right) A_{X X} & 0 \\
0 & 0
\end{array}\right] \cdot\left[\begin{array}{c}
\hat{X}(k \mid k) \\
\hat{Z}(k \mid k)
\end{array}\right]+\left[\begin{array}{c}
K_{F} \cdot y(k+1) \\
Z(k+1)
\end{array}\right]} \\
& +\left[\begin{array}{c}
\left(I_{n X}-K_{F} C_{y X}\right) \\
0
\end{array}\right]\left(B_{X} \Delta u(k)+B w_{X} \Delta w(k)\right) \\
& {\left[\begin{array}{c}
\hat{X}(k+1 \mid k+1) \\
\hat{Z}(k+1 \mid k+1)
\end{array}\right]=\left[\begin{array}{c}
\left(I_{n X}-K_{F} C_{y X}\right) A_{X X} \hat{X}(k \mid k)+K_{F} y(k+1)+\left(I_{n X}-K_{F} C_{y X}\right)\left(B_{X} \Delta u(k)+B w_{X} \Delta w(k)\right) \\
Z(k+1)
\end{array}\right]}
\end{aligned}
$$

A expressão final para a estimação dos estados utilizando (4-16) mostra que o estado $Z$ é diretamente medido, diferentemente do que ocorre com o estado $X$. A vantagem dessa expressão é oferecer ao controlador uma atualização mais rápida dos estados $Z$. Contudo, o comportamento esperado desse observador é bastante sensível a alterações bruscas nesses estados, portanto seu uso não é recomendável quando o sinal medido das entradas for muito ruidoso. 


\section{ANÁLISE DE ESTABILIDADE DO CONTROLADOR PROPOSTO}

$\mathrm{Na}$ formulação em dois passos, graças à inclusão das variáveis de folga na formulação, obtêm-se dois problemas de otimização que serão sempre viáveis pois essas slacks $\left(\delta_{u, k} \mathrm{e}\right.$ $\left.\delta_{y, k}\right)$ são irrestritas. Contudo, não há garantia a priori de que o controlador será estável. É possível prová-lo admitindo-se que o sistema já se encontra num estado em que os modos integradores podem ser zerados com as ações de controle previstas em um horizonte de controle, ou seja $x^{i}\left(k+m+\theta_{\max } \mid k\right)=0$.

A metodologia empregada consiste em mostrar primeiramente que uma vez calculada uma solução ótima num instante $\mathrm{k}$, existe uma solução viável no instante posterior k+1 de maneira que a função-objetivo não aumenta. Além disso, pode-se mostrar que a seqüência $V_{2 b, k}$ é estritamente decrescente quando $V_{2 b, k} \neq 0$ e portanto converge assintoticamente para 0 .

A demonstração deste fato repousa fundamentalmente na hipótese de que o modelo empregado pelo controlador reproduz com exatidão o comportamento da planta na ausência de distúrbios não medidos. Assim, caso os distúrbios medidos mantenham-se constantes, temos

$$
\hat{x}(k+j \mid k)=\hat{x}(k+j \mid k+1), \forall j \geq 1
$$

A validade de (5-1) decorre do seguinte: no instante k, o controlador estima, a partir de seu modelo e do estado conhecido $x(k)$, o estado futuro $\hat{x}(k+1 \mid k)$, através de (3-31). Porém, após a implementação da primeira ação de controle calculada, a evolução do sistema real também é dada por (3-8), de maneira que $\hat{x}(k+1 \mid k)=x(k+1)$. Para os instantes posteriores, (5-1) será verdadeira apenas se as ações de controle usadas na predição dos estados futuros em $\mathrm{k}+1$ forem exatamente aquelas calculadas no instante $\mathrm{k}$.

Isto posto, mostrar a recursividade da solução não é um grande obstáculo. Seja $\Delta u_{k}^{b^{*}}, y_{s p, k}^{*}$, $\delta_{u, k}^{*}, \delta_{y, k}^{*}$ a solução ótima de P2b calculada no instante k e seja $\Delta u_{k}^{a^{*}}$ a solução ótima de P2a calculada no mesmo instante k. O símbolo $\Delta u_{k}^{b^{*}}$ representa um vetor com as ações de controle calculadas, ou seja, 


$$
\Delta u_{k}^{b^{*}}=\left[\begin{array}{c}
\Delta u^{b^{*}}(k \mid k) \\
\Delta u^{b^{*}}(k+1 \mid k) \\
\vdots \\
\Delta u^{b^{*}}(k+m-1 \mid k)
\end{array}\right]
$$

A solução ótima é, em particular, viável. Então se tem, trivialmente, que ela satisfaz as restrições, ou seja, para o problema P2a:

$$
\begin{gathered}
-\Delta u_{\max } \leq \Delta u^{a^{*}}(k+j \mid k) \leq \Delta u_{\max }, j=0,1, \ldots, m-1 \\
u_{\min } \leq u^{a^{*}}(k+j \mid k) \leq u_{\max }, j \in \mathbb{N}
\end{gathered}
$$

Para o problema $\mathrm{P} 2 \mathrm{~b}$,

$$
\begin{gathered}
-\Delta u_{\max } \leq \Delta u^{b^{*}}(k+j \mid k) \leq \Delta u_{\max }, j=0,1, \ldots, m-1 \\
u_{\min } \leq u^{b^{*}}(k+j \mid k) \leq u_{\max }, j \in \mathbb{N} \\
u^{b^{*}}(k+m-1 \mid k)-u_{d e s}-\delta_{u, k}=0 \\
y_{\min } \leq y_{s p, k}^{*} \leq y_{\max } \\
x^{s}\left(k+m+\theta_{\max } \mid k\right)-y_{s p, k}^{*}-\delta_{y, k}^{*}=0 ; \\
N_{i} A^{\theta_{\max }} \operatorname{Co\Delta }_{u_{k}^{a^{*}}}=N_{i} A^{\theta_{\max }} \operatorname{Co\Delta }_{u_{k}^{b^{*}}}
\end{gathered}
$$

No instante $\mathrm{k}+1$, propõe-se como solução o conjunto $\Delta \tilde{u}_{k+1}^{a}, \Delta \tilde{u}_{k+1}^{b}, \tilde{y}_{s p, k+1}, \tilde{\delta}_{u, k+1}, \tilde{\delta}_{y, k+1}$, herdado da solução anterior da seguinte forma:

$$
\Delta \tilde{u}_{k+1}^{a}=\left[\begin{array}{c}
\Delta u^{b^{*}}(k+1 \mid k) \\
\Delta u^{b^{*}}(k+2 \mid k) \\
\vdots \\
0
\end{array}\right]
$$




$$
\begin{gathered}
\Delta \tilde{u}_{k+1}^{b}=\left[\begin{array}{c}
\Delta u^{b^{*}}(k+1 \mid k) \\
\Delta u^{b^{*}}(k+2 \mid k) \\
\vdots \\
0
\end{array}\right] \\
\tilde{y}_{s p, k+1}=y_{s p, k}^{*} \\
\tilde{\delta}_{y, k+1}=\delta_{y, k}^{*} \\
\tilde{\delta}_{u, k+1}=\delta_{u, k}^{*}
\end{gathered}
$$

A solução herdada consiste, portanto, em aplicar em $k+1$ a mesma seqüência de ações de controle calculada em $k$, além de manter o valor das variáveis de folga e de $y_{s p}$. Desta maneira, ocorre a situação descrita pela equação (5-1), em que a predição dos estados em um instante continua válida no instante posterior.

Com essa proposta de solução, observa-se que as restrições (3-44) e (3-45), que dizem respeito às entradas do sistema respeitarem os limites e variações admissíveis, serão obedecidas pela solução herdada. Portanto, a existência de uma solução ótima para P2a no instante $k$ implica na existência de pelo menos uma solução viável em $k+1$.

Da mesma forma, como (3-47) e (3-48) são análogas a (3-44) e (3-45), também serão satisfeitas no instante $k+1$ pela solução herdada. No entanto, para provar a factibilidade recursiva do problema $\mathrm{P} 2 \mathrm{~b}$, é preciso analisar com mais detalhe as demais restrições.

A restrição (3-49) escrita no instante $k+1$ será igual a

$$
u(k)+\sum_{j=0}^{m-1} \Delta \tilde{u}^{b}(k+j \mid k+1)-u_{d e s}-\tilde{\delta}_{u, k+1}=a
$$

sendo a uma constante. Ora, $u(k)=u(k-1)+\Delta u^{b^{*}}(k \mid k)$. Além disso, $\sum_{j=0}^{m-1} \Delta \tilde{u}^{b}(k+1+j \mid k+1)=\sum_{j=1}^{m-1} \Delta u^{b^{*}}(k+j \mid k)$ pela forma com que é construída a solução herdada. Logo, (5-13) é equivalente a 


$$
\begin{aligned}
& u(k-1)+\Delta u^{b^{*}}(k \mid k)+\sum_{j=0}^{m-1} \Delta \tilde{u}^{b}(k+1+j \mid k+1)-u_{d e s}-\tilde{\delta}_{u, k+1}=a \\
& u(k-1)+\sum_{j=0}^{m-1} \Delta u^{b^{*}}(k+j \mid k)-u_{d e s}-\tilde{\delta}_{u, k+1}=a
\end{aligned}
$$

Supôs-se que $\tilde{\delta}_{u, k+1}=\delta_{u, k}^{*}$. Então, desde que $u_{\text {des }}$ não seja alterado entre k e k+1, tem-se que o primeiro membro de (5-14) é idêntico ao primeiro membro de (5-4), portanto $a=0$ e esta restrição será atendida pela nova solução.

Finalmente, pode-se mostrar que a restrição (3-51) será satisfeita pela solução herdada usando-se o mesmo tipo de raciocínio. Escrevendo-a no instante $\mathrm{k}+1$,

$$
x^{s}\left(k+m+\theta_{\max }+1 \mid k+1\right)-\tilde{y}_{s p, k+1}-\tilde{\delta}_{y, k+1}=b
$$

Usando o modelo para relacionar a predição em estado estacionário da saída $\left(x^{s}\right)$ nos instantes $k$ e $k+1$,

$$
x^{s}(k+1)=I_{n y} \cdot x^{s}(k)+\Delta t I_{n i}^{*} \cdot x^{i}(k)+\sum_{l=0}^{\theta_{\max }} B_{l}^{s} \cdot \Delta u(k-l)
$$

Particularizando para os instantes $k+m+\theta_{\text {max }}$ e $k+m+\theta_{\max }+1$ :

$$
\begin{aligned}
& x^{s}\left(k+m+\theta_{\max }+1 \mid k\right)=I_{n y} \cdot x^{s}\left(k+m+\theta_{\max } \mid k\right)+\Delta t I_{n i}^{*} \cdot x^{i}\left(k+m+\theta_{\max } \mid k\right) \\
& +\sum_{l=0}^{\theta_{\max }} B_{l}^{s} \cdot \Delta u\left(k+m+\theta_{\max }-l \mid k\right)
\end{aligned}
$$

As ações de controle são todas iguais a zero nesse intervalo e portanto o somatório não interfere no resultado. Substituindo de (5-17) em (5-15):

$$
I_{n y} \cdot x^{s}\left(k+m+\theta_{\max } \mid k\right)+\Delta t I_{n i}^{*} \cdot x^{i}\left(k+m+\theta_{\max } \mid k\right)-\tilde{y}_{s p, k+1}-\tilde{\delta}_{y, k+1}=b
$$

Lembrando-se que $\tilde{y}_{s p, k+1}=y_{s p, k}^{*}$ e $\tilde{\delta}_{y, k+1}=\delta_{y, k}^{*}$, a última equação é equivalente a 


$$
I_{n y} \cdot x^{s}\left(k+m+\theta_{\max } \mid k\right)+\Delta t I_{n i}^{*} \cdot x^{i}\left(k+m+\theta_{\max } \mid k\right)-y_{s p, k}^{*}-\delta_{y, k}^{*}=b
$$

Porém, usando o fato de que a solução no instante k era viável, como explicitado em (5-6), é possível reduzir essa equação a

$$
I_{n i}^{*} \cdot x^{i}\left(k+m+\theta_{\max } \mid k\right)=b
$$

Por hipótese, $x^{i}\left(k+m+\theta_{\max } \mid k\right)=0$ e conseqüentemente $b=0$. Resta apenas mostrar que a restrição (3-52) também é respeitada pelas soluções herdadas. Isso no entanto é trivial pela forma com que essas soluções são definidas, isto é, como $\Delta \tilde{u}_{k+1}^{a}=\Delta \tilde{u}_{k+1}^{b}$, então as predições para $x^{i}$ nos problemas P2a e P2b tem que ser iguais. Logo, conclui-se que a solução herdada é realmente uma solução factível para o problema.

Da comparação entre as funções objetivo nos instantes $k$ e $k+1$ (com a solução ótima e a solução herdada, respectivamente), tem-se:

$$
V_{2 b, k}^{*}-\tilde{V}_{2 b, k+1}=\left\|y(k / k)-y_{s p, k}^{*}-\delta_{y, k}^{*}\right\|_{Q_{y}}^{2}+\left\|u(k / k)-u_{d e s}-\delta_{u, k}^{*}\right\|_{Q_{u}}^{2}+\left\|\Delta u^{*}(k / k)\right\|_{R}^{2}
$$

Como as matrizes $Q_{y}, Q_{u}, R$, são definidas positivas, a diferença $V_{2 b, k}^{*}-\tilde{V}_{2 b, k+1}$ será igual a zero se e somente se o sistema estiver em estado estacionário. Caso contrário, $V_{2 b, k}^{*}-\tilde{V}_{2 b, k+1}>0 \Rightarrow \tilde{V}_{2 b, k+1}<V_{2 b, k}^{*}$. Como a solução herdada é apenas uma solução possível e não necessariamente a ótima, tem-se que o cálculo da solução ótima em k+1 produzirá um novo resultado para a função objetivo de maneira que $V_{2 b, k+1}^{*} \leq \tilde{V}_{2 b, k+1}<V_{2 b, k}^{*}$. Como $\mathrm{V}_{2 \mathrm{~b}}$ é ilimitada, conclui-se que é uma função de Lyapunov e o sistema é assintoticamente estável. No entanto, duas situações possíveis podem ocorrer com relação ao estado estacionário alcançado pela planta: todas as saídas encontram-se dentro de suas respectivas faixas ou ao menos uma saída está fora da faixa. A primeira circunstância corresponde à configuração em que as entradas atingem seus targets de maneira que os valores correspondentes das saídas estejam dentro das faixas. Nesse caso, é evidente que os termos $T_{1}$ e $T_{2}$ da função objetivo definida em (3-64) são iguais a zero pois as saídas estão dentro da faixa. Analogamente, $T_{3}$ é igual a zero pois as entradas atingem seus targets. $\mathrm{O}$ termo $T_{4}$ é sempre nulo como 
demonstrado anteriormente, enquanto $T_{5}$ é zero em qualquer estado estacionário. Por fim, as contribuições das slacks são iguais a zero pois as condições propostas para entradas e saídas foram alcançáveis. Isso garante que, nesse caso, o ponto de ótimo global do problema de otimização corresponde a um valor 0 da função objetivo. Vale ressaltar que esse ponto não é único já que a variação de uma saída no interior de sua faixa não produz nenhum impacto na função objetivo.

O caso em que os targets e as faixas das saídas são incompatíveis pode acontecer devido a imprecisões do modelo de otimização responsável pela determinação desses valores. Quando se tem condições operacionais em que ou todas as saídas estão dentro de suas faixas ou todas as entradas atingem seus targets, de modo mutuamente exclusivo, pode-se deduzir que alguma parcela dos termos $T_{1}, T_{2}$ ou $T_{3}$ contribuirá com uma quantidade positiva à função custo, além do acréscimo referente à presença de algum valor diferente de zero nas slacks (já que nem todas as restrições originais podem ser respeitadas simultaneamente). Assim, o valor ótimo global de $V_{2 b, k}$ será estritamente maior do que 0 . O estado estacionário alcançado será aquele em que os desvios das entradas e saídas com relação aos valores especificados sejam mínimos conforme a ponderação decorrente das matrizes $Q_{y}, Q_{u}, S_{y}$ e $S_{u}$. 


\section{SIMULAÇÕES E ANÁLISES NUMÉRICAS}

\subsection{ESTUDO DE CASO BASEADO NUM SISTEMA REAL}

Para avaliar numericamente a performance do controlador proposto, foram realizadas simulações a partir de um modelo de uma planta de produção de óxido de etileno. A Figura 6-1, extraída de Carrapiço (2004) representa esquematicamente o processo:

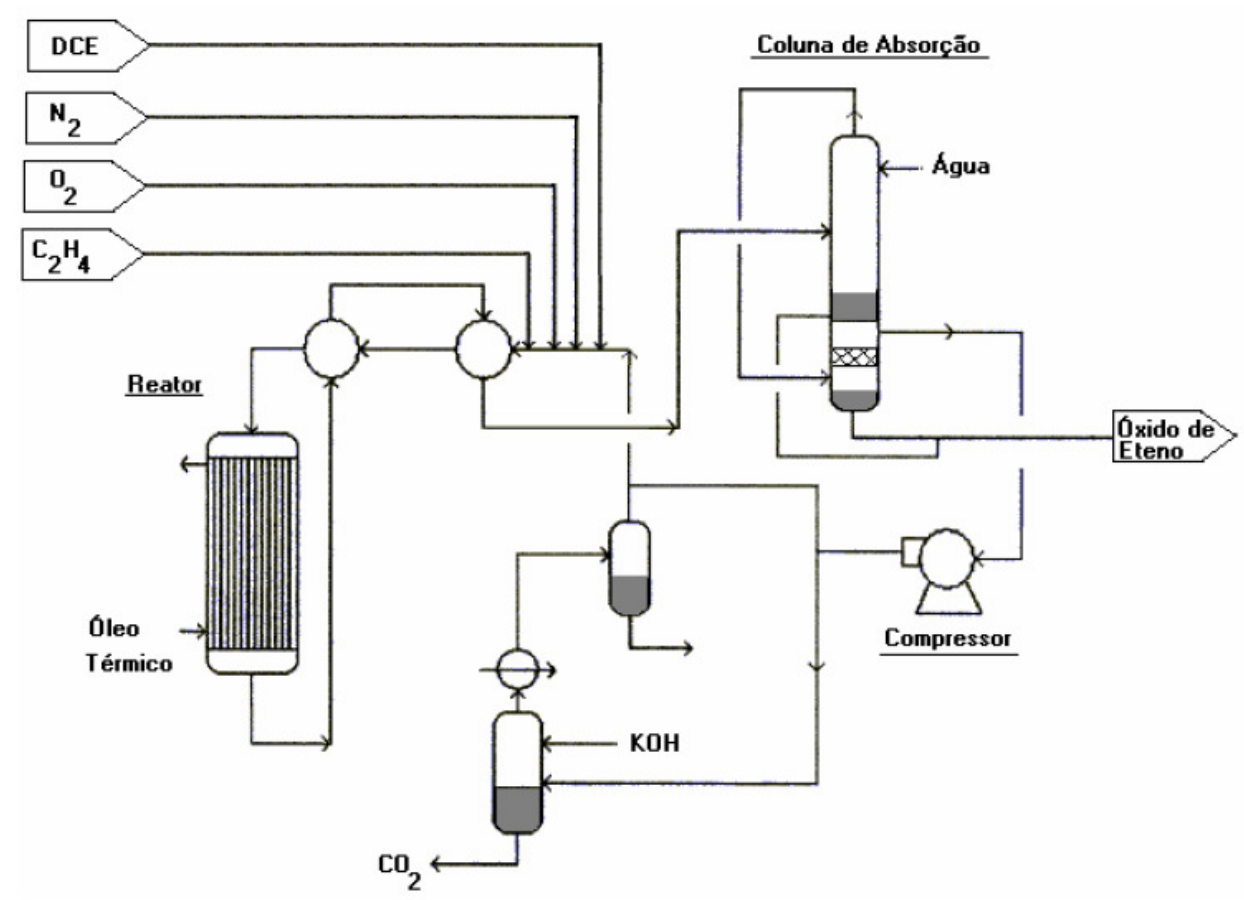

Figura 6-1: Visão geral de uma planta de óxido de etileno - Carrapiço (2004)

Maiores detalhes sobre o processo podem ser vistos em Carrapiço (2004) e Rodrigues e Odloak (2003). Um modelo para esse sistema considerando 4 saídas (variáveis controladas) e 4 entradas (variáveis manipuladas) é apresentado em Carrapiço (2004). As variáveis consideradas são apresentadas nas tabelas 6-1 e 6-2: 
Tabela 6-1: Conjunto de variáveis controladas

\begin{tabular}{ccc}
\hline & Variáveis Controladas & Unidade \\
\hline$y_{1}$ & Teor de $\mathrm{O}_{2}$ no gás de reciclo na entrada do reator & $\%$ \\
$y_{2}$ & Teor de $\mathrm{C}_{2} \mathrm{H}_{4}$ no gás de reciclo na entrada do reator & $\%$ \\
$y_{3}$ & Temperatura do óleo térmico na entrada do reator & ${ }^{\circ} \mathrm{C}$ \\
$y_{4}$ & Pressão do gás de reciclo na entrada do reator & $\mathrm{kgf} / \mathrm{cm}^{2}$ \\
\hline
\end{tabular}

Tabela 6-2: Conjunto de variáveis manipuladas

\begin{tabular}{ccc}
\hline & Variáveis Manipuladas & Unidade \\
\hline$u_{1}$ & Vazão de entrada de oxigênio no reator & $\mathrm{kg} / \mathrm{h}$ \\
$u_{2}$ & Vazão de entrada de etileno no reator & $\mathrm{kg} / \mathrm{h}$ \\
$u_{3}$ & Abertura da válvula pequena de óleo térmico & $\%$ \\
$u_{4}$ & Vazão de entrada de nitrogênio no reator & $\mathrm{kg} / \mathrm{h}$ \\
\hline
\end{tabular}

O modelo identificado em termos de função de transferência em tempo contínuo para esse sistema foi:

$$
G(s)=\left[\begin{array}{cccc}
\frac{-10^{-4}(-95 s+1) e^{-s}}{32,16 s^{2}+4,65 s+1} & \frac{-2,3 \cdot 10^{-3}}{s} & \frac{-3,2 \cdot 10^{-3}(-s+1) e^{-2 s}}{64,55 s^{2}+8,83 s+1} & \frac{-7,5 \cdot 10^{-6}}{s} \\
\frac{-1,69 \cdot 10^{-4} e^{-3 s}}{s} & \frac{2,1 \cdot 10^{-4} e^{-8 s}}{s} & \frac{-1,9 \cdot 10^{-3}(1,47 s+1)}{9,67 s^{2}+13,55 s+1} & \frac{-1,07 \cdot 10^{-4}}{s} \\
\frac{8,1 \cdot 10^{-3}(-0,02 s+1) e^{-4 s}}{52,45 s^{2}+11,92 s+1} & \frac{-5,5 \cdot 10^{-5} e^{-15 s}}{s} & \frac{9,6 \cdot 10^{-3}(s+1) e^{-2 s}}{54,42 s^{2}+6,58 s+1} & \frac{-2,53 \cdot 10^{-3} e^{-10 s}}{s} \\
\frac{-3,9 \cdot 10^{-5} e^{-4 s}}{s} & \frac{5,7 \cdot 10^{-5} e^{-8 s}}{s} & \frac{-1,4 \cdot 10^{-3}(s+1)}{8,67 s^{2}+14,48 s+1} & \frac{-7,6 \cdot 10^{-5} e^{-6 s}}{s}
\end{array}\right]
$$

Todas as saídas apresentam funções de transferência integradoras com relação, pelo menos, às vazões de etileno e nitrogênio. Nota-se ainda que os tempos mortos são significativos, atingindo até 15 minutos no caso da função de transferência que relaciona a temperatura do óleo térmico e a vazão de etileno. As relações entrada-saída que não são integradoras foram representadas por funções de transferência de segunda ordem, portanto $n a=2$. Não foram considerados distúrbios medidos. A dimensão do estado $X$ é igual a $n y+n d+n y=4+4 \cdot 4 \cdot 2+4=40$ e do estado $Z$ é $n u \cdot \theta_{\max }=4 \cdot 15=60$, totalizando 100 componentes para o estado completo $x$. 
Apesar da pequena dimensão do caso tratado e da estrutura de um problema de programação quadrática não ser extremamente complexa para tratamento computacional, notou-se que em algumas situações ocorria um mau condicionamento do problema, levando o solver inicialmente utilizado (rotina de programação quadrática do MATLAB ${ }^{\circledR}$ ) a identificar erroneamente algumas instâncias como infactíveis, apesar da presença de variáveis de folga que garantem a viabilidade em todos os instantes.

Para contornar problemas numéricos, optou-se por uma implementação do problema de otimização na plataforma GAMS ${ }^{\circledR}$, utilizando-se CONOPT como solver. Graças a essa mudança de algoritmo, todas as instâncias simuladas puderam ser resolvidas adequadamente, comprovando que se tratava apenas de erros numéricos e não de formulação.

\subsection{COMPARAÇÃO ENTRE AS FORMULAÇÕES COM 1 E 2 PASSOS}

A formulação desenvolvida anteriormente permite que se escolham zonas para as saídas e targets para as entradas. Os valores escolhidos para a simulação estão apresentados nas Tabelas 6-3 a 6-5:

Tabela 6-3: Faixas desejáveis para as saídas

\begin{tabular}{ccc}
\hline Variável Controlada & Valor mínimo & Valor máximo \\
\hline Teor de $\mathrm{O}_{2}$ no gás de reciclo & $6 \%$ & $6,5 \%$ \\
Teor de $\mathrm{C}_{2} \mathrm{H}_{4}$ no gás de reciclo & $12 \%$ & $19,7 \%$ \\
Temperatura do óleo térmico & $270^{\circ} \mathrm{C}$ & $320^{\circ} \mathrm{C}$ \\
Pressão do gás de reciclo & $16 \mathrm{kgf} / \mathrm{cm}^{2}$ & $20 \mathrm{kgf} / \mathrm{cm}^{2}$ \\
\hline
\end{tabular}

Tabela 6-4: Valores desejáveis para as entradas

\begin{tabular}{lcccc}
\hline \multicolumn{1}{c}{ Variáveis Manipuladas } & $\begin{array}{c}\text { Valor } \\
\text { mínimo }\end{array}$ & $\begin{array}{c}\text { Valor } \\
\text { máximo }\end{array}$ & Target & $\begin{array}{c}\text { Variação } \\
\text { máxima }\end{array}$ \\
\hline Vazão de entrada de oxigênio no reator & $5700 \mathrm{~kg} / \mathrm{h}$ & $6900 \mathrm{~kg} / \mathrm{h}$ & $6210 \mathrm{~kg} / \mathrm{h}$ & $25 \mathrm{~kg} / \mathrm{h}$ \\
Vazão de entrada de etileno no reator & $4500 \mathrm{~kg} / \mathrm{h}$ & $5700 \mathrm{~kg} / \mathrm{h}$ & $5612 \mathrm{~kg} / \mathrm{h}$ & $25 \mathrm{~kg} / \mathrm{h}$ \\
Abertura da válvula pequena de óleo & $0 \%$ & $100 \%$ & $50 \%$ & $2 \%$ \\
térmico & & & & \\
Vazão de entrada de nitrogênio no & $25 \mathrm{~kg} / \mathrm{h}$ & $95 \mathrm{~kg} / \mathrm{h}$ & $75 \mathrm{~kg} / \mathrm{h}$ & $10 \mathrm{~kg} / \mathrm{h}$ \\
reator & & & & \\
\hline
\end{tabular}


Tabela 6-5: Valores iniciais

\begin{tabular}{|c|c|c|c|}
\hline Variáveis Controladas & Valor inicial & Variáveis Manipuladas & Valor inicial \\
\hline Teor de $\mathrm{O}_{2}$ no gás de reciclo & $5,7 \%$ & $\begin{array}{l}\text { Vazão de entrada de } \\
\text { oxigênio no reator }\end{array}$ & $6300 \mathrm{~kg} / \mathrm{h}$ \\
\hline $\begin{array}{c}\text { Teor de } \mathrm{C}_{2} \mathrm{H}_{4} \text { no gás de } \\
\text { reciclo }\end{array}$ & $21 \%$ & $\begin{array}{l}\text { Vazão de entrada de } \\
\text { etileno no reator }\end{array}$ & $5600 \mathrm{~kg} / \mathrm{h}$ \\
\hline Temperatura do óleo térmico & $268^{\circ} \mathrm{C}$ & $\begin{array}{l}\text { Abertura da válvula } \\
\text { pequena de óleo térmico }\end{array}$ & $60 \%$ \\
\hline Pressão do gás de reciclo & $19,5 \mathrm{kgf} / \mathrm{cm}^{2}$ & $\begin{array}{l}\text { Vazão de entrada de } \\
\text { nitrogênio no reator }\end{array}$ & $42 \mathrm{~kg} / \mathrm{h}$ \\
\hline
\end{tabular}

O tempo de amostragem considerado na simulação foi equivalente a 1 minuto, de modo que todos os tempos mortos da planta fossem múltiplos desse valor. Para horizonte de controle foi escolhido $m=7$, tendo sido este valor determinado através de tentativa e erro.

Num primeiro momento, a sintonia foi feita de forma a considerar apenas as faixas das saídas, ou seja, a matriz $\mathrm{Q}_{\mathrm{u}}$ de pesos dos targets de entradas foi colocada igual a 0. Como o sistema possui o mesmo número de entradas e saídas, há graus de liberdade suficientes para levar todas as saídas a suas faixas. A sintonia utilizada foi determinada através de tentativa e erro e é apresentada na Tabela 6-6:

\begin{tabular}{cc} 
Tabela 6-6: Sintonia utilizada sem considerar targets nas entradas \\
\hline Parâmetro de sintonia & Valor escolhido \\
\hline$Q_{y}$ & $\operatorname{diag}\left(\left[\begin{array}{llll}2 \cdot 10^{3} & 5 \cdot 10^{2} & 5 \cdot 10^{2} & 5 \cdot 10^{1}\end{array}\right]\right)$ \\
$Q_{i}$ & $\operatorname{diag}\left(\left[\begin{array}{llll}10^{3} & 10^{3} & 10^{2} & 10^{1}\end{array}\right]\right)$ \\
$Q_{u}$ & $\operatorname{diag}\left(\left[\begin{array}{llll}0 & 0 & 0 & 0\end{array}\right]\right)$ \\
$R$ & $\operatorname{diag}\left(\left[\begin{array}{llll}10^{-1} & 10^{-1} & 10^{1} & 10^{1}\end{array}\right]\right)$ \\
$\bar{R}^{v}$ & $\operatorname{diag}\left(\left[\begin{array}{llll}10^{-5} & 10^{-5} & 10^{-5} & 10^{-5}\end{array}\right]\right)$ \\
$S_{y}$ & $\operatorname{diag}\left(\left[\begin{array}{llll}2 \cdot 10^{5} & 5 \cdot 10^{4} & 5 \cdot 10^{4} & 5 \cdot 10^{3}\end{array}\right]\right)$ \\
$S_{u}$ & $\operatorname{diag}\left(\left[\begin{array}{lllll}0 & 0 & 0 & 0\end{array}\right]\right)$ \\
$S_{i}$ & $\operatorname{diag}\left(\left[\begin{array}{llll}10^{3} & 10^{3} & 10^{2} & 10^{1}\end{array}\right]\right)$ \\
\hline
\end{tabular}

Os resultados das simulações estão nas Figuras 6-2 a 6-4: 

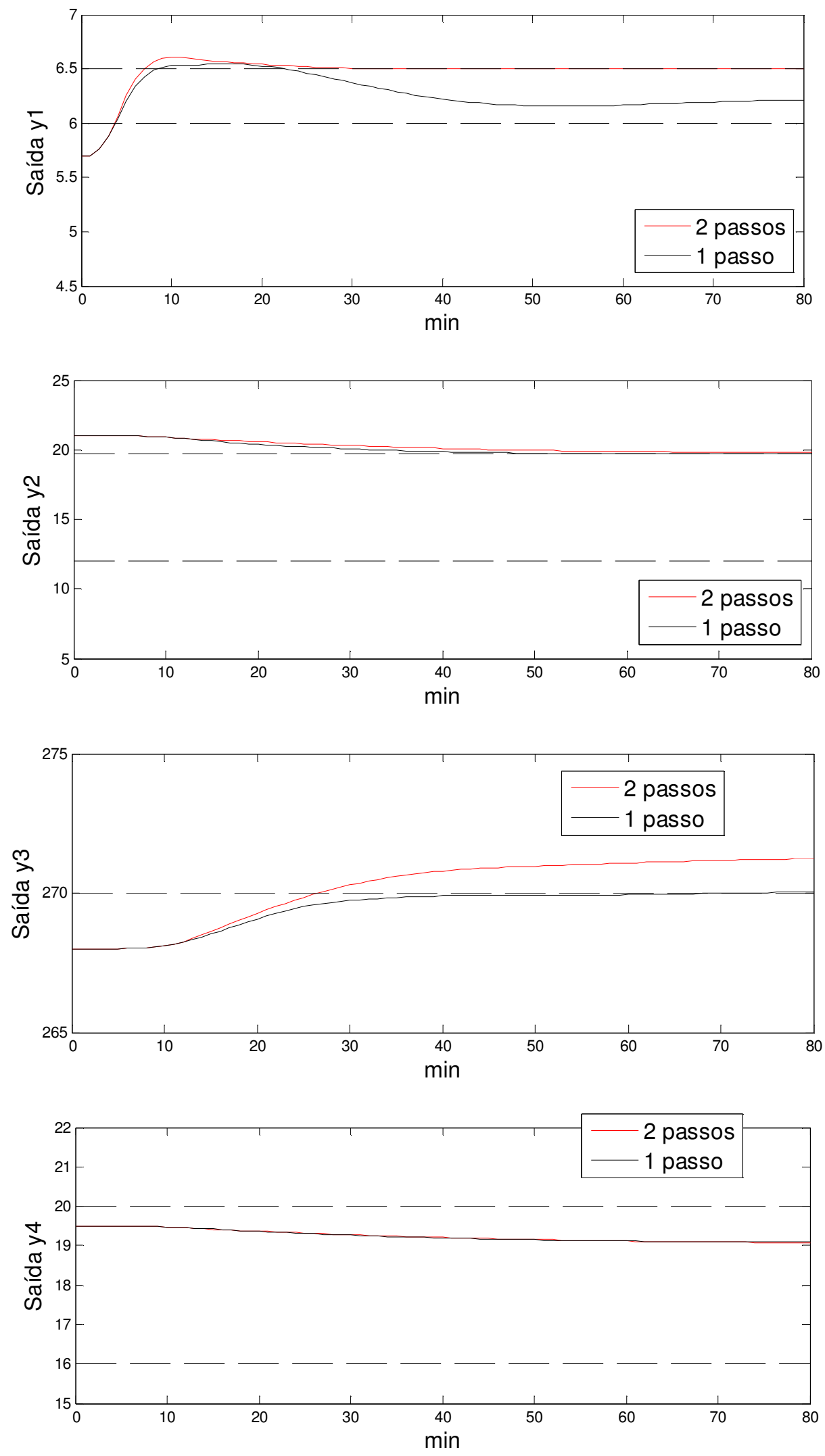

Figura 6-2: Evolução das saídas do sistema - sem target nas entradas 

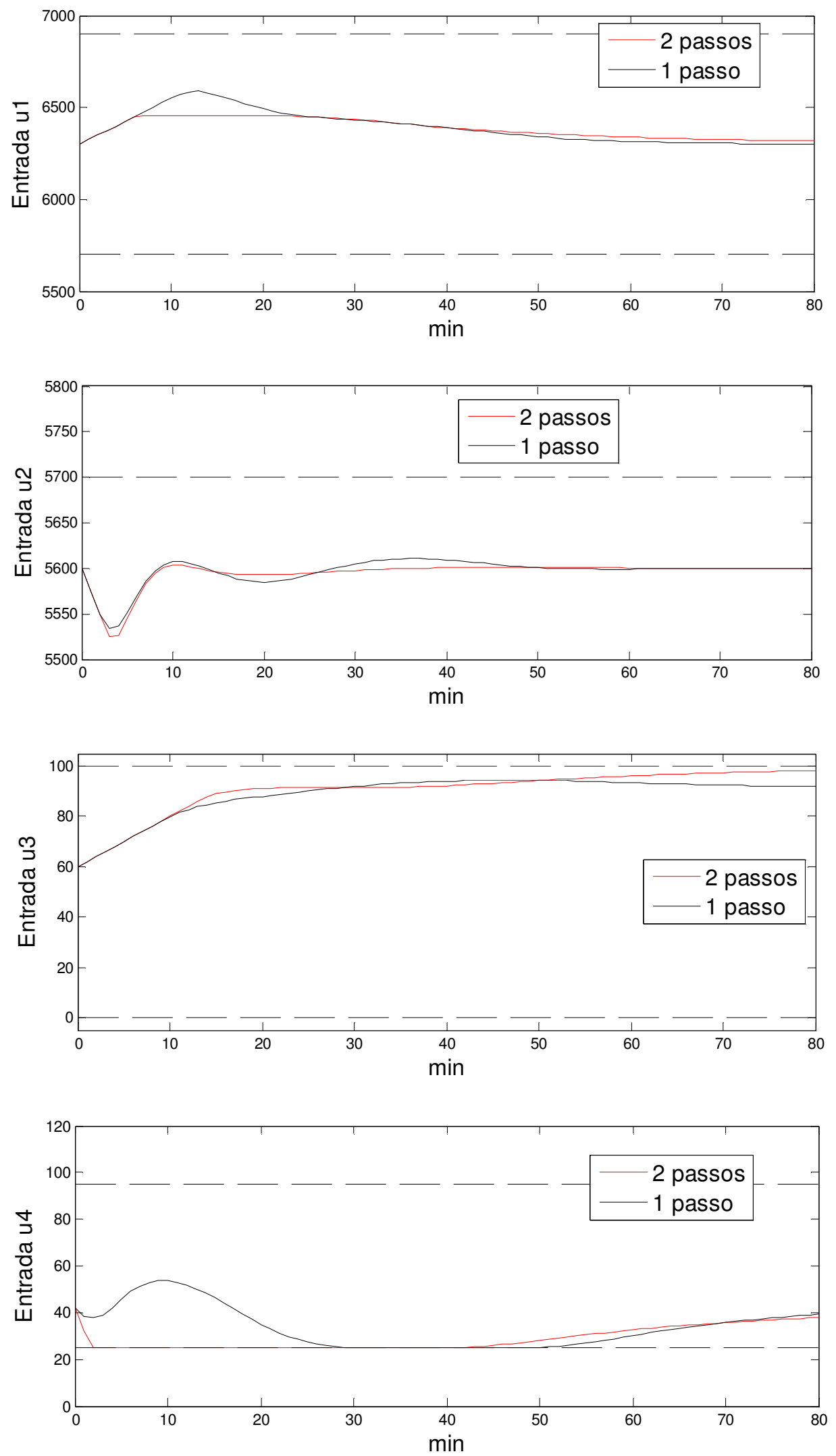

Figura 6-3: Evolução das entradas do sistema - sem target nas entradas 


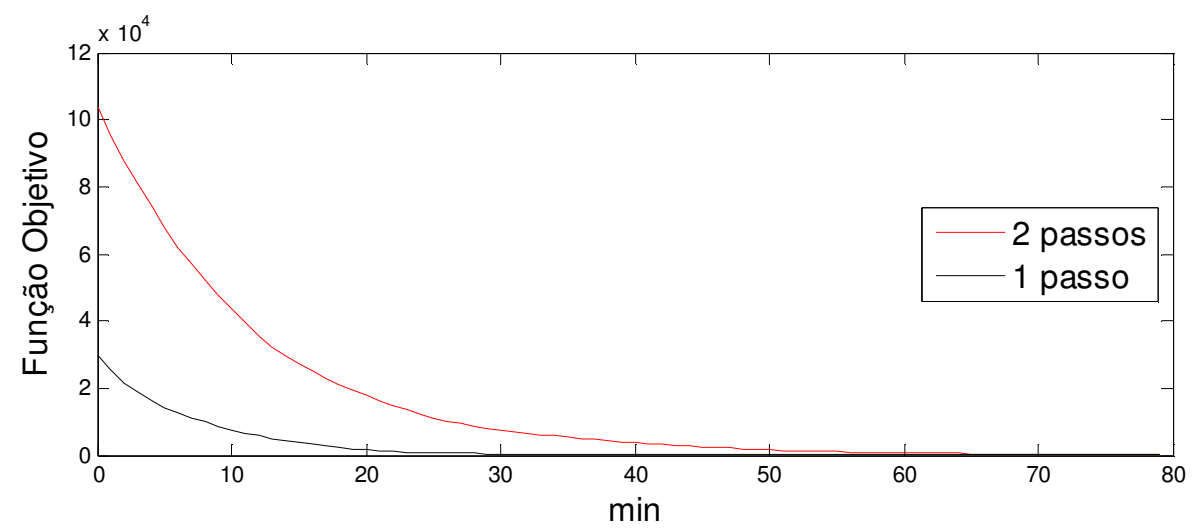

Figura 6-4: Função objetivo - sem targets nas entradas

A análise dos resultados obtidos quando não há targets nas entradas mostra que as saídas que estavam fora da faixa tendem a alcançar essas regiões desejadas. Em ambas as formulações, todas as saídas de fato foram conduzidas às suas faixas dentro do horizonte da simulação (80 períodos de amostragem, equivalente a 80 minutos). Como não há especificações sobre as entradas, ambas as funções objetivo atingiriam o valor 0 se a simulação fosse mais longa. Nota-se que a função objetivo calculada pelo controlador com algoritmo em um passo é menor do que aquela do controlador com algoritmo em dois passos. Neste exemplo, tem-se portanto que o desempenho do controlador foi prejudicado pela quebra artificial em dois passos.

Posteriormente, pode-se analisar o comportamento quando se deseja levar simultaneamente as entradas a seus targets. Neste exemplo, a especificação de targets e zonas não é consistente, ou seja, não é possível alcançar todos os valores desejados de entradas e saídas. Por não haver graus de liberdade suficientes, o sistema atinge um estado estacionário em que a função objetivo é diferente de zero. O valor estacionário das variáveis controladas e manipuladas depende fortemente da ponderação relativa entre elas. A sintonia utilizada nesse caso foi definida através de tentativa e erro e é apresentada na Tabela 6-7:

Tabela 6-7: Sintonia utilizada considerando targets nas entradas

\begin{tabular}{clll}
\hline Parâmetro de sintonia & \multicolumn{3}{c}{ Valor escolhido } \\
\hline$Q_{y}$ & $\operatorname{diag}\left(\left[\begin{array}{llll}2 \cdot 10^{4} & 2 \cdot 10^{4} & 1 \cdot 10^{4} & 1 \cdot 10^{4}\end{array}\right]\right)$ \\
$Q_{i}$ & $\operatorname{diag}\left(\left[\begin{array}{llll}10^{4} & 10^{3} & 10^{2} & 10^{1}\end{array}\right]\right)$ \\
$Q_{u}$ & $\operatorname{diag}\left(\left[\begin{array}{llll}10^{-1} & 10^{1} & 10^{1} & 10^{1}\end{array}\right]\right)$ \\
\hline
\end{tabular}

(Continua) 
(Conclusão)

\begin{tabular}{ll}
$R$ & $\operatorname{diag}\left(\left[\begin{array}{llll}10^{-1} & 10^{-1} & 10^{1} & 10^{1}\end{array}\right]\right)$ \\
$\bar{R}^{v}$ & $\operatorname{diag}\left(\left[\begin{array}{llll}10^{-5} & 10^{-5} & 10^{-5} & 10^{-5}\end{array}\right]\right)$ \\
$S_{y}$ & $\operatorname{diag}\left(\left[\begin{array}{llll}2 \cdot 10^{6} & 2 \cdot 10^{6} & 1 \cdot 10^{6} & 1 \cdot 10^{6}\end{array}\right]\right)$ \\
$S_{u}$ & $\operatorname{diag}\left(\left[\begin{array}{llll}10^{-1} & 10^{1} & 10^{1} & 10^{1}\end{array}\right]\right)$ \\
$S_{i}$ & $\operatorname{diag}\left(\left[\begin{array}{llll}10^{4} & 10^{3} & 10^{2} & 10^{1}\end{array}\right]\right)$ \\
\hline
\end{tabular}

Os resultados das simulações estão nas Figuras 6-5 a 6-7:
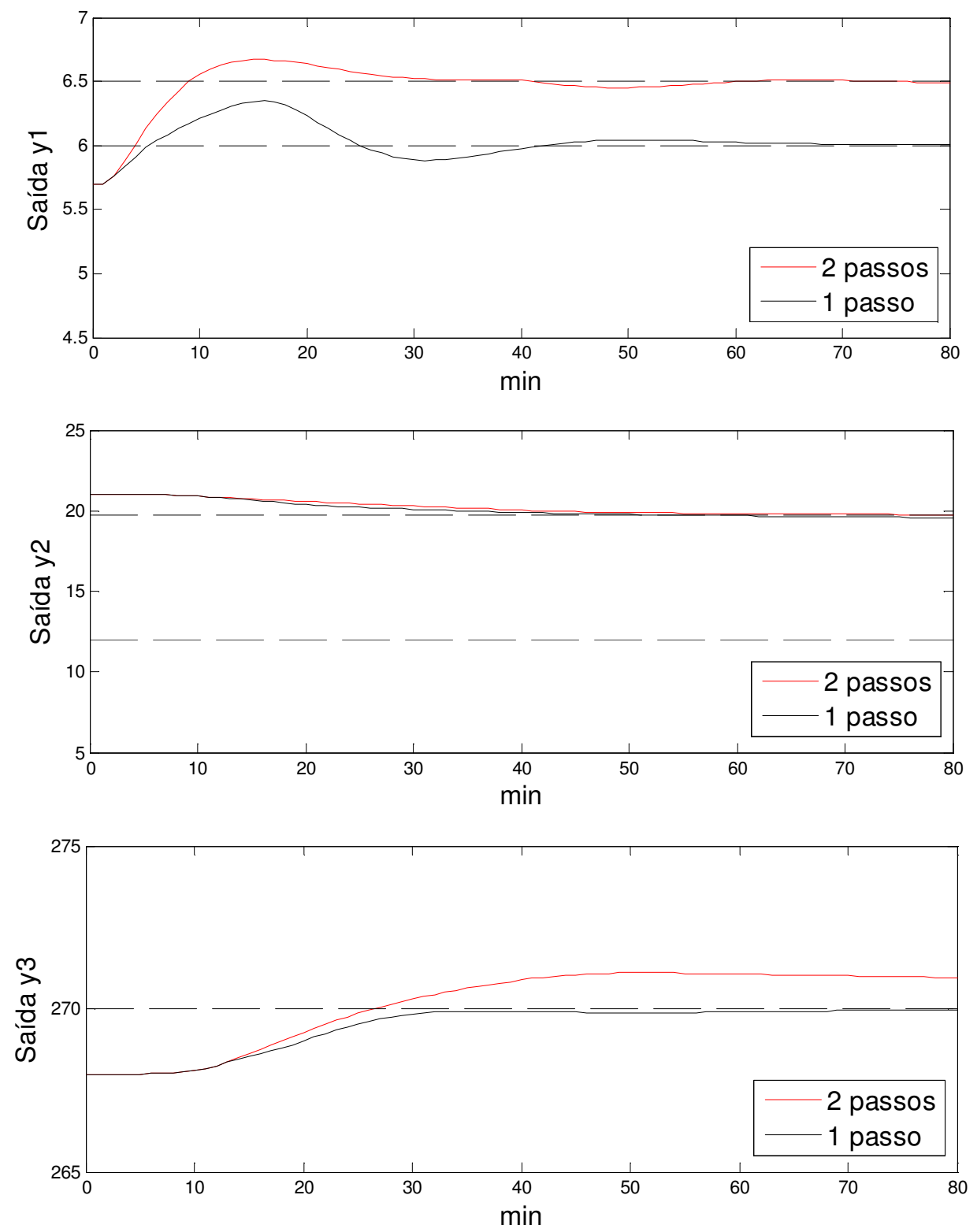


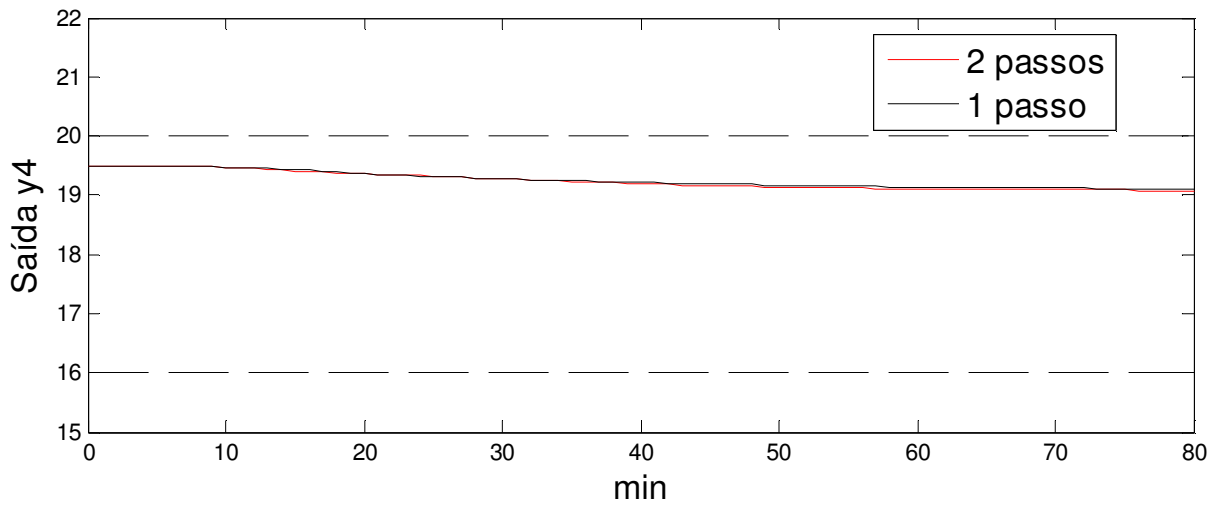

Figura 6-5: Evolução das saídas do sistema - com target nas entradas
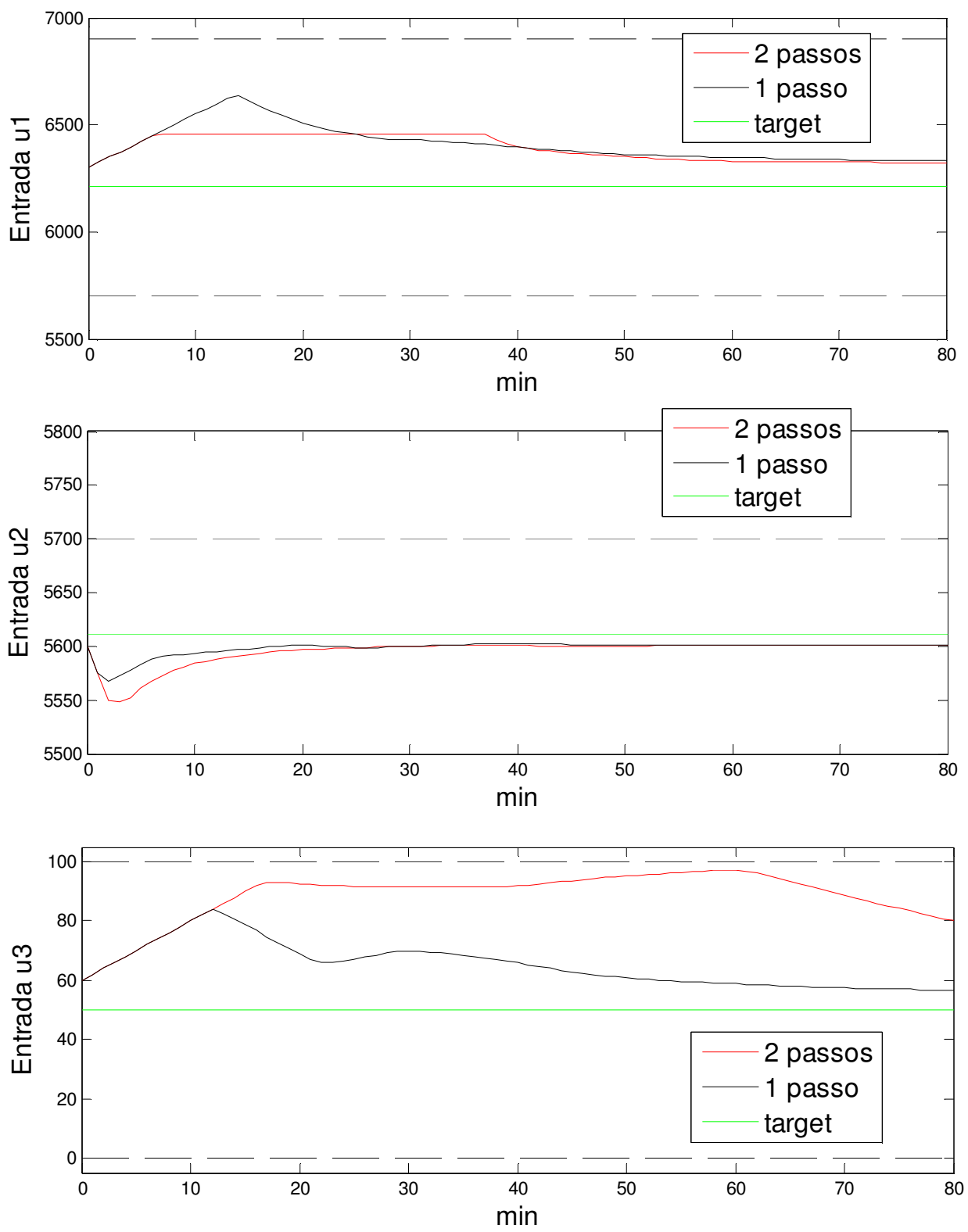


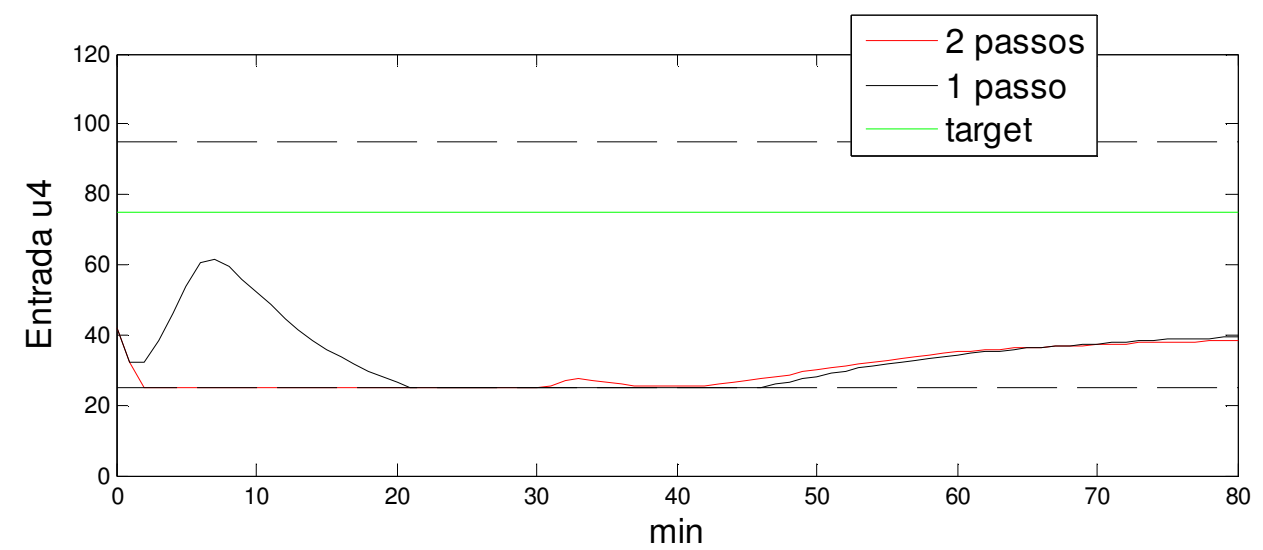

Figura 6-6: Evolução das entradas do sistema - com target nas entradas
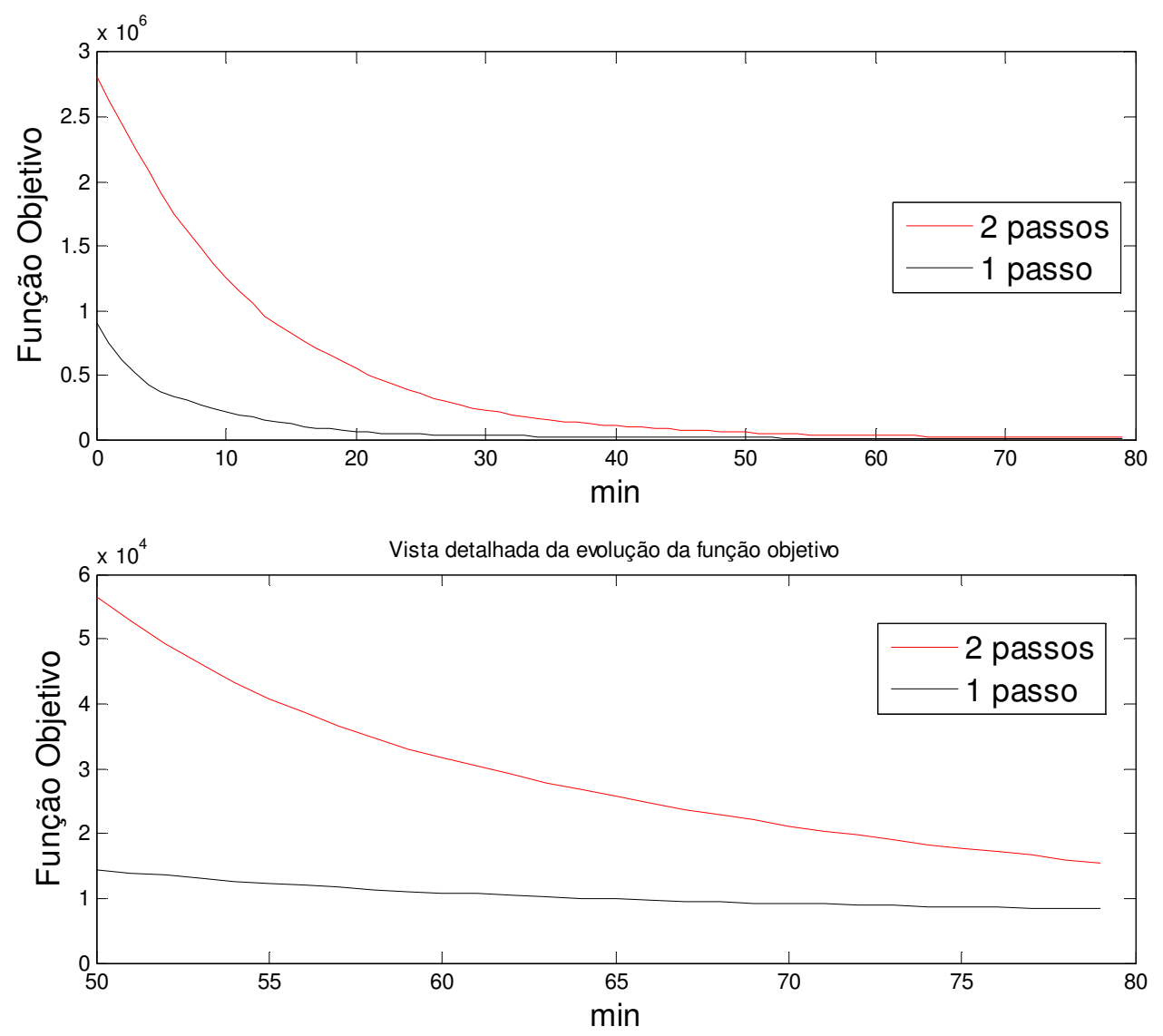

Figura 6-7: Função objetivo - com targets nas entradas

Quando se incorporam targets nas entradas, é possível levar a planta a condições operacionais diferentes, desde que se utilize uma sintonia adequada. No presente exemplo, foi dada prioridade às saídas do sistema. Nota-se que todas as saídas alcançam suas faixas dentro do horizonte de simulação. Por outro lado, nenhuma das entradas atinge seu target previamente especificado. 
Além disso, ressalta-se que a função objetivo em ambos os casos convergiu para um valor diferente de zero, como era de se esperar devido à especificação inconsistente de targets e zonas. Novamente a performance do controlador com a formulação em 1 passo foi melhor, atingindo valores menores para a função objetivo e alcançando um estado estacionário em menos tempo.

\subsection{COMPARAÇÃO ENTRE OBSERVADOR HEURÍSTICO E FILTRO DE KALMAN}

\subsubsection{Caso 1: comparação entre o Filtro de Kalman e estado completamente medido}

Sabe-se que no caso de aplicações reais de controladores preditivos é necessário empregar um observador de estados. $\mathrm{O}$ desempenho do controlador é degradado quando há a introdução de um observador, em relação à situação em que o estado é completamente medido. Foram realizadas simulações comparando-se o comportamento em malha fechada quando o estado é medido ou apenas estimado. Para simplificar as comparações, será utilizado apenas o algoritmo do controlador com problema decomposto em 2 passos.

Escolheu-se como estado inicial da planta um vetor em que todas as componentes, com exceção de $x^{s}$, eram iguais a 0 . Para $x^{s}$, definiu-se o valor igual às condições iniciais apresentadas na Tabela 6-5. Por outro lado, a estimativa inicial usada pelo controlador para o estado da planta era igual a 0 para todas as componentes.

A sintonia utilizada foi definida após tentativa e erro e é apresentada na Tabela 6-8:

\begin{tabular}{cc} 
Tabela 6-8: Sintonia utilizada considerando medição completa do estado \\
\hline Parâmetro de sintonia & $\operatorname{diag}\left(\left[\begin{array}{llll}10^{5} & 9 \cdot 10^{3} & 6 \cdot 10^{4} & 1 \cdot 10^{4}\end{array}\right]\right)$ \\
\hline$Q_{y}$ & $\operatorname{diag}\left(\left[\begin{array}{llll}8 \cdot 10^{5} & 10^{4} & 10^{3} & 10^{2}\end{array}\right]\right)$ \\
$Q_{i}$ & $\operatorname{diag}\left(\left[\begin{array}{llll}10^{-3} & 10^{-3} & 1 & 1\end{array}\right]\right)$ \\
$Q_{u}$ & $\operatorname{diag}\left(\left[\begin{array}{llll}10^{1} & 10^{1} & 10^{2} & 10^{2}\end{array}\right]\right)$ \\
$R$ & $\operatorname{diag}\left(\left[\begin{array}{llll}10^{-4} & 10^{-4} & 10^{-4} & 10^{-4}\end{array}\right]\right)$ \\
$\bar{R}^{v}$ & $\operatorname{diag}\left(\left[\begin{array}{llll}10^{7} & 9 \cdot 10^{5} & 6 \cdot 10^{6} & 1 \cdot 10^{6}\end{array}\right]\right)$ \\
$S_{y}$ & $\operatorname{diag}\left(\left[\begin{array}{llll}10^{-3} & 10^{-3} & 1 & 1\end{array}\right]\right)$ \\
$S_{u}$ &
\end{tabular}


O horizonte de controle é idêntico ao utilizado na seção 6.2, ou seja, $m=7$. Os resultados obtidos estão apresentados nas Figuras de 6-8 a 6-10:
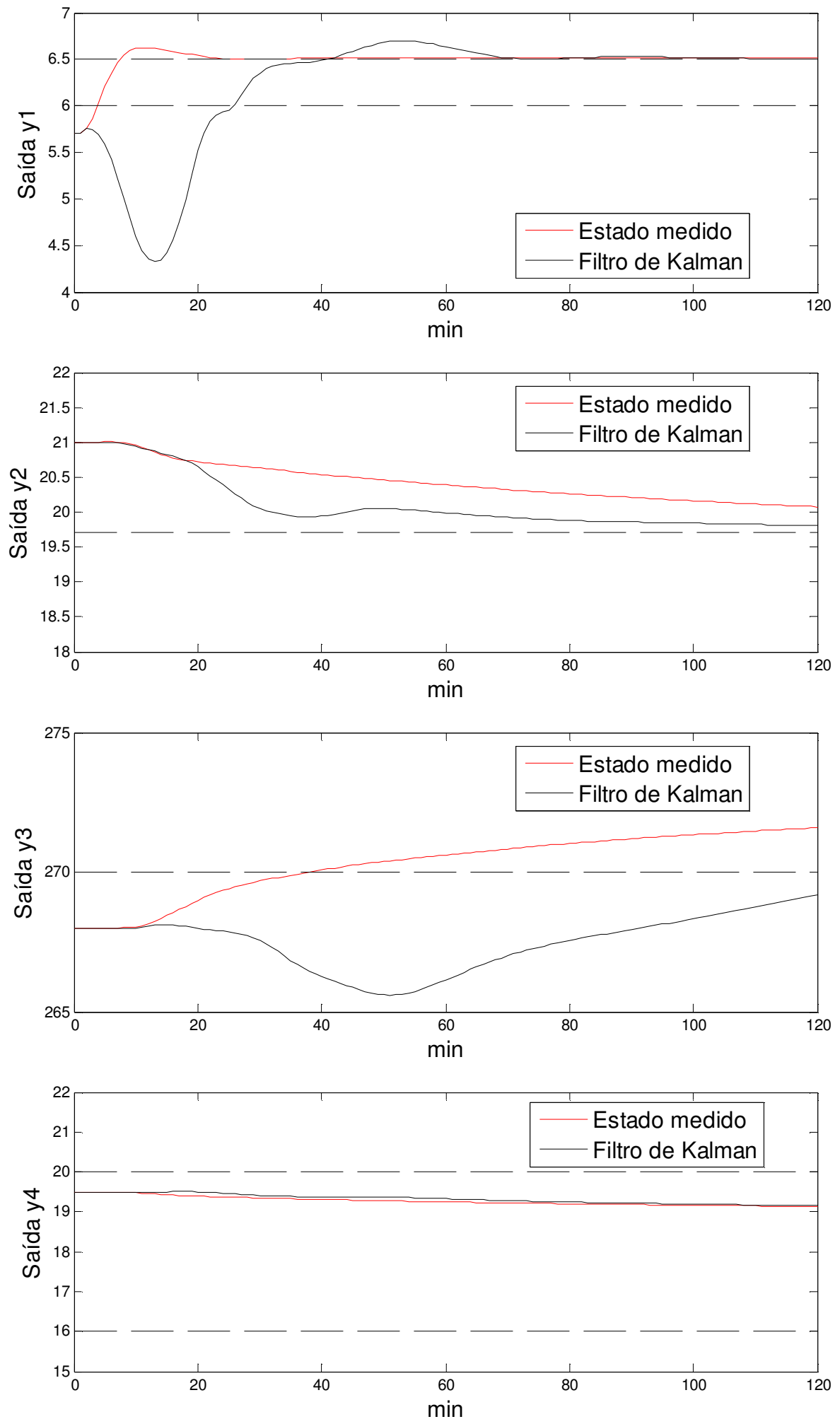

Figura 6-8: Evolução das entradas do sistema com medição do estado ou Filtro de Kalman 

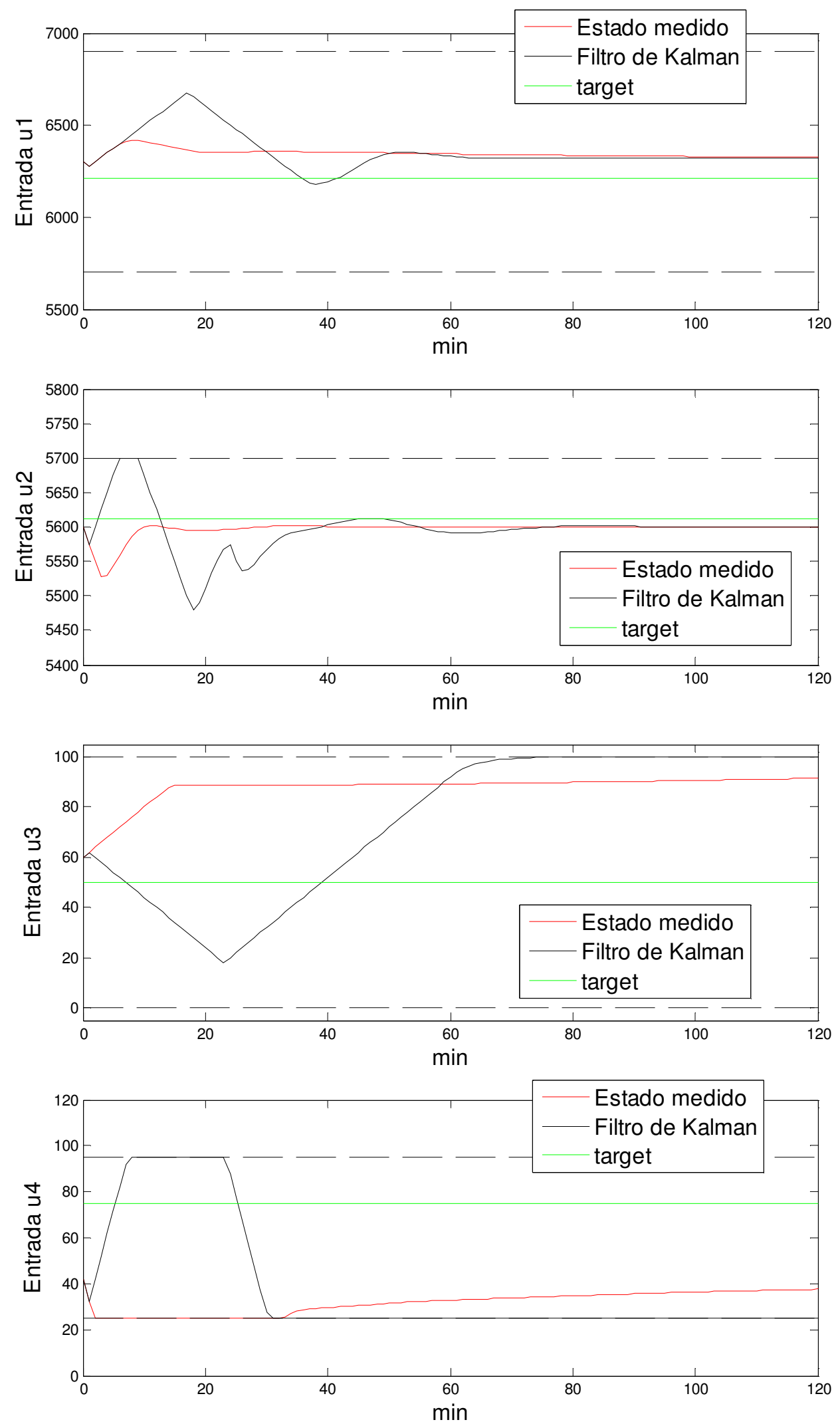

Figura 6-9: Evolução das saídas do sistema com medição do estado ou Filtro de Kalman 

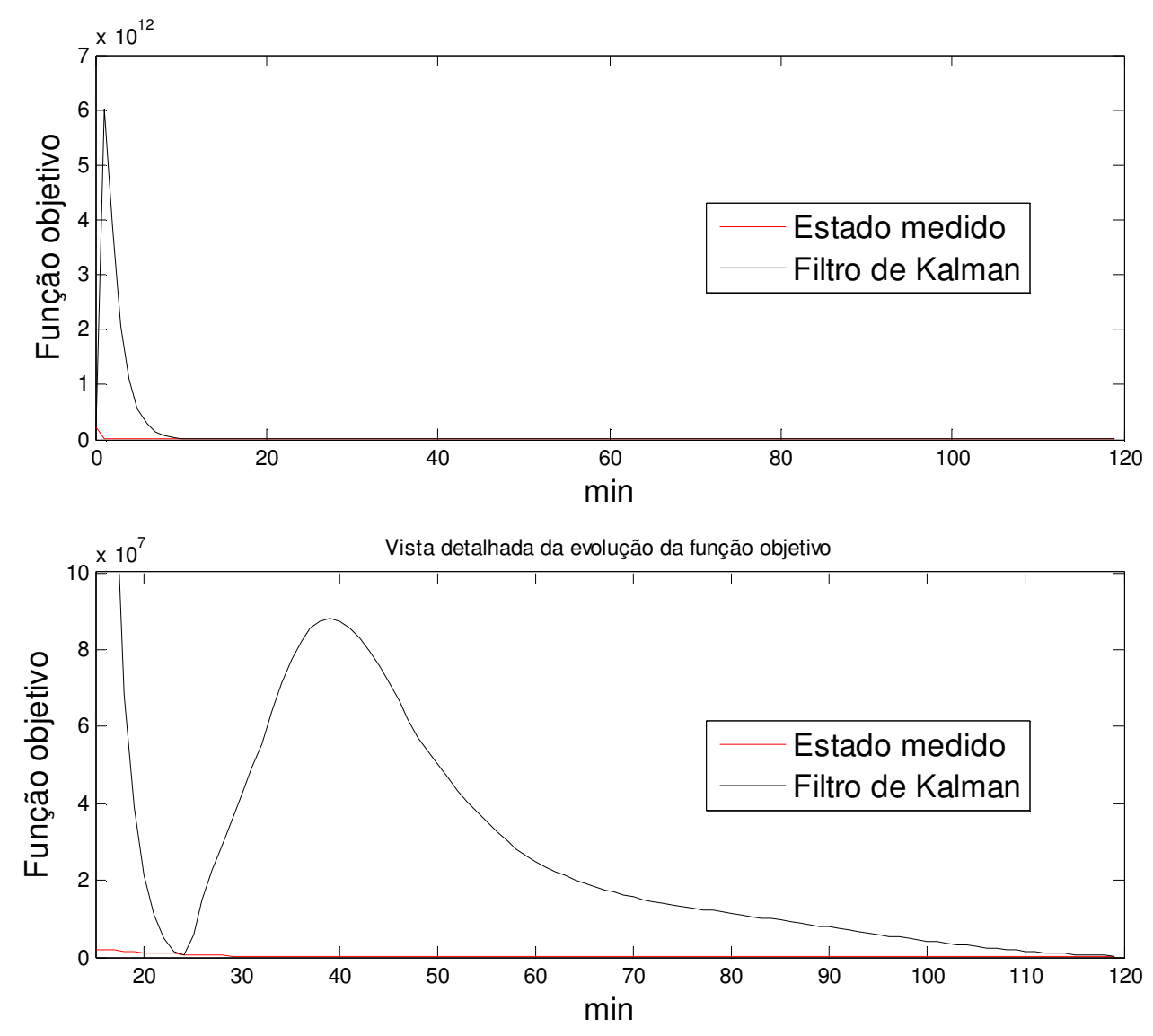

Figura 6-10: Evolução da função objetivo com medição do estado ou Filtro de Kalman

O comportamento das entradas e saídas no Caso 1 mostra como a estimativa pode interferir no desempenho do controlador. A saída $y_{1}$ (teor de oxigênio na entrada do reator) é uma variável determinante para a rentabilidade do processo industrial. Se o estado pudesse ser medido, essa variável controlada alcançaria o estado estacionário desejado em cerca de 25 minutos. Contudo, com a presença do Filtro de Kalman esse tempo eleva-se a cerca de 70 minutos. Com relação à saída $y_{3}$ (temperatura do óleo na camisa do reator), nota-se que o controlador que utiliza a estimativa do Filtro de Kalman não conseguiu alcançar a faixa desejada dentro do horizonte de 120 minutos, enquanto isso teria sido feito em apenas 40 minutos caso o estado fosse mensurável.

O desempenho pior do controlador que utiliza o filtro é retratado pelas funções objetivo: a partir de 20 minutos de simulação, o valor para o controlador com um hipotético estado medido é uma ou duas ordens de grandeza menor do que o controlador real com Filtro de Kalman. Um processo de sintonia das matrizes de covariância dos ruídos poderia levar a uma 
resposta mais adequada do Filtro, porém trata-se de um ajuste que deve ser feito caso a caso e sem garantias formais de sucesso.

\subsubsection{Caso 2: comparação entre o Filtro de Kalman e o observador heurístico com modelo nominal}

O principal objetivo do observador proposto é aumentar a velocidade de convergência do erro de estimação, o que é feito com a diminuição do módulo dos autovalores da matriz $(I-L C) A$, em que $L$ é o ganho do observador. Na Figura 6-11, pode-se comprovar essa redução:

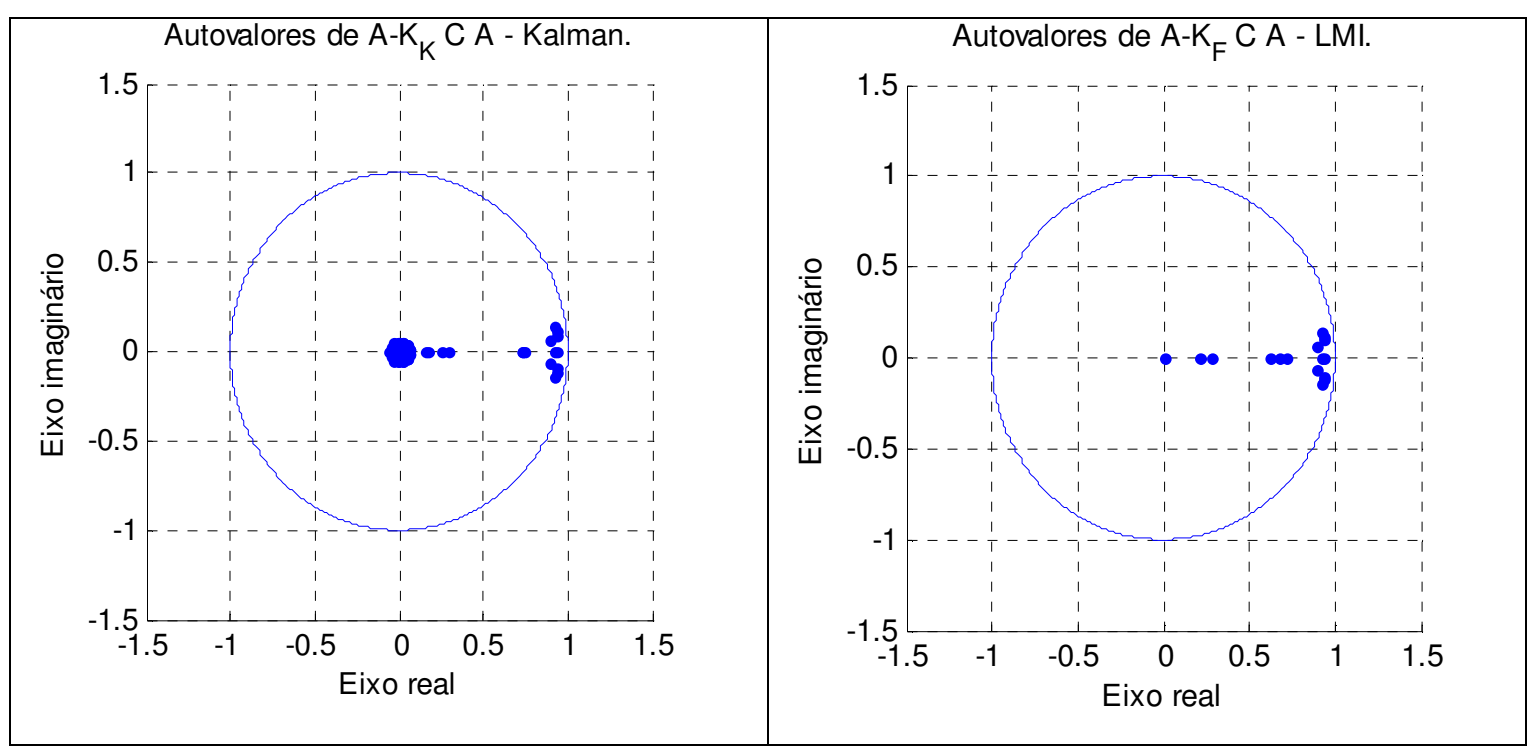

Figura 6-11: Comparação entre os autovalores das matrizes de transição dos erros de estimação

Nota-se que os autovalores próximos à origem presentes na matriz com o ganho do Filtro de Kalman foram levados a zero. Além disso, a soma do módulo de todos os autovalores diminuiu de 3,32 para 3,25. Porém, os autovalores de maior módulo na matriz obtida com o Filtro de Kalman continuam presentes na matriz oriunda do observador heurístico.

A maior velocidade de convergência no caso determinístico foi comprovada através de simulação. De modo idêntico ao Caso 1, considerou-se que a planta estava em estado estacionário, situação em que a componente $x^{s}$ é igual ao valor inicial das saídas e as demais componentes são iguais a zero. A estimativa inicial utilizada pelo controlador também foi a mesma do Caso 1, ou seja, igual a zero em todas as componentes. 
Simulou-se o comportamento do sistema em malha fechada mas impondo-se que as ações de controle seriam iguais a 0 durante todo o horizonte analisado. Desse modo, o estado da planta permaneceria constante e o estado estimado tenderia a alcançá-lo. Esse comportamento está retratado na Figura 6-12:
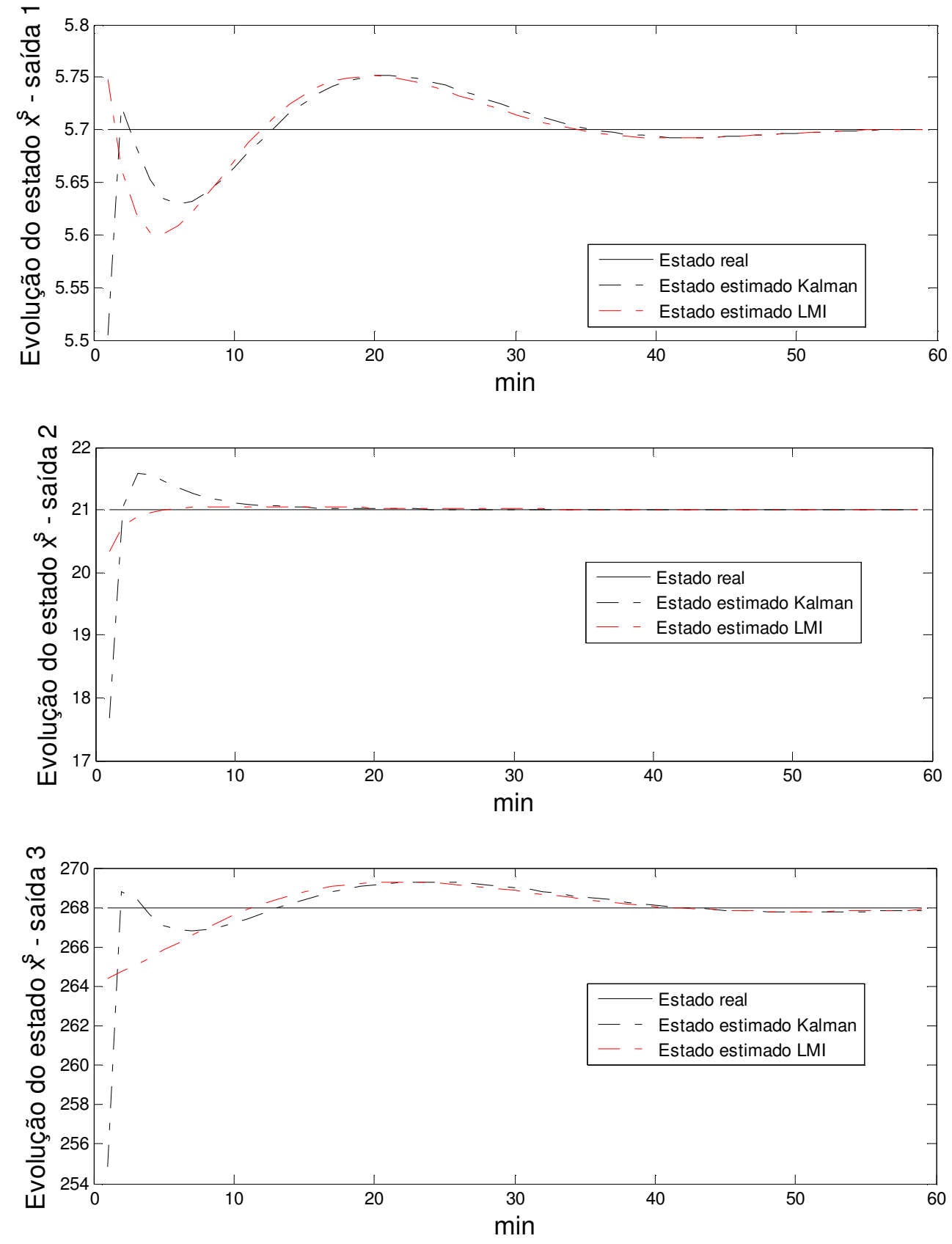


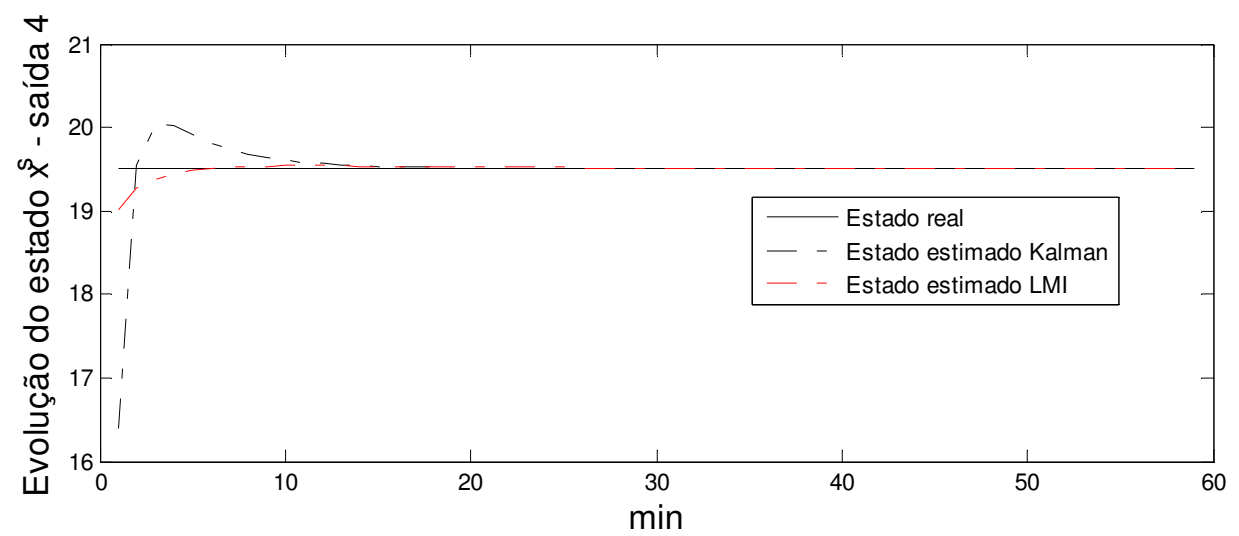

Figura 6-12: Evolução da estimação do estado $x^{s}$

Os estados $x^{2}(k), x^{3}(k)$ e $x^{4}(k)$, referentes às saídas $y_{2}, y_{3}$ e $y_{4}$, respectivamente, alcançam os estados reais em menos tempo e sem oscilações quando utilizado o observador heurístico. Contudo, no caso do estado $x^{1}(k)$, correspondente a $y_{1}$, há menos oscilações quando é utilizado o Filtro de Kalman.

Para avaliar a diferença de desempenho em malha fechada conforme o observador utilizado, é necessário simular situações em que o estado estimado seja diferente do estado real do sistema. Neste Caso 2 utilizaram-se as mesmas condições iniciais do Caso 1, ou seja, admitiuse que a planta estava em estado estacionário, situação em que a componente $x^{s}$ é igual ao valor inicial das saídas e as demais componentes são iguais a zero. Com relação ao observador, definiu-se que a estimativa inicial por ele utilizada seria a origem. A dinâmica da planta é suposta conhecida exatamente, portanto a diferença entre o estado real e o estimado provém unicamente do desconhecimento, por parte do observador, do estado inicial real.

Ao longo da simulação, a estimação de estados é feita seguindo a equação

$$
\begin{aligned}
& \hat{x}(k+1 \mid k)=A \hat{x}(k \mid k)+B \Delta u(k) \\
& \hat{x}(k+1 \mid k+1)=\hat{x}(k+1 \mid k)+L(y(k+1)-C \hat{x}(k+1 \mid k))
\end{aligned}
$$

em que $L$, o ganho do observador, corresponde ao ganho do filtro de Kalman ou ao do observador heurístico.

Para simplificar as comparações, estão ilustrados a seguir apenas os resultados obtidos quando o controlador resolve o problema de otimização em dois passos. A sintonia utilizada é a mesma do Caso 1, já apresentada na Tabela 6-8. O horizonte de controle também é $m=7$. 
Os resultados obtidos podem ser vistos nas Figuras 6-13 a 6-15:
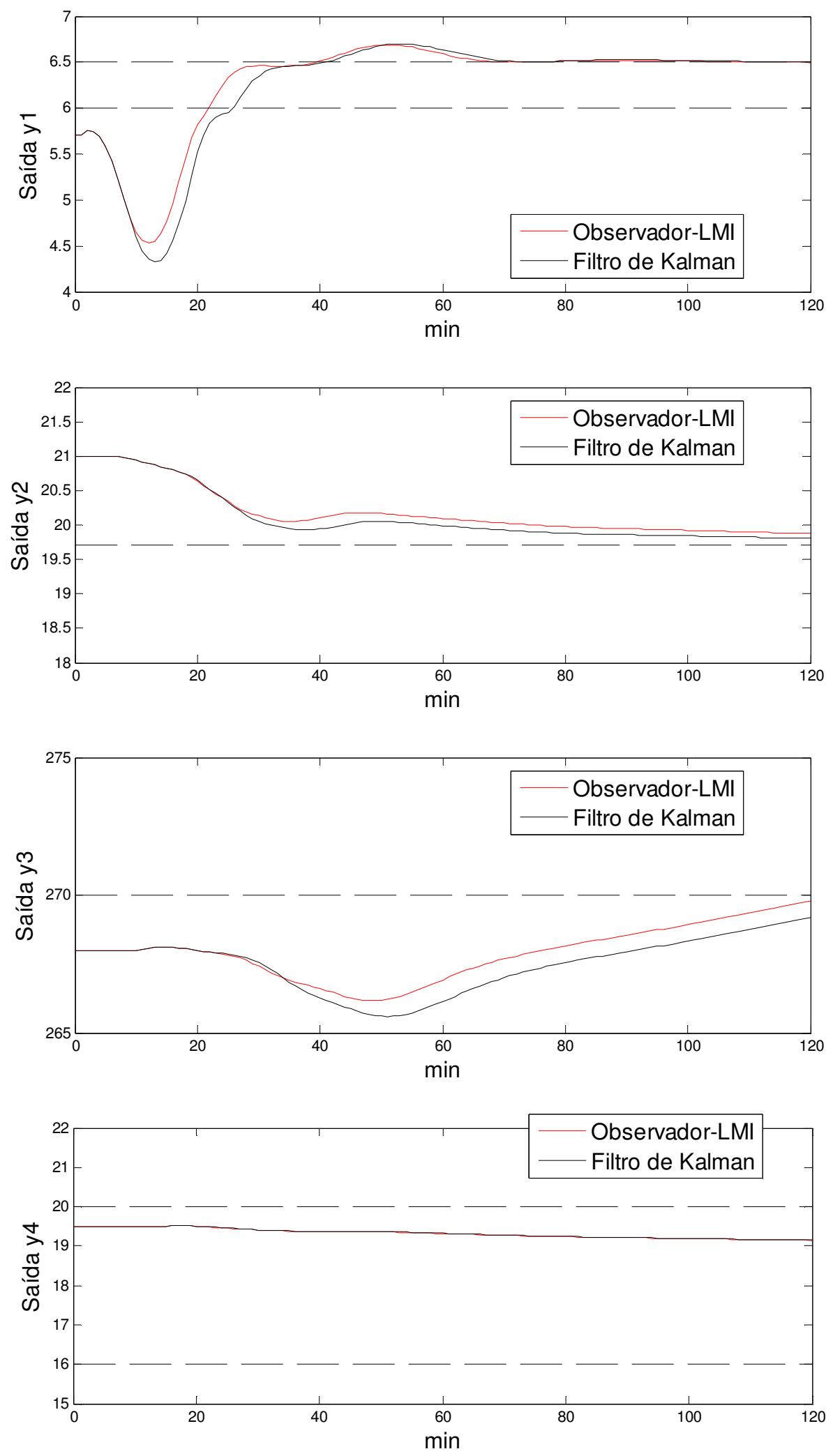

Figura 6-13 : Evolução das saídas quando o estado inicial é desconhecido 

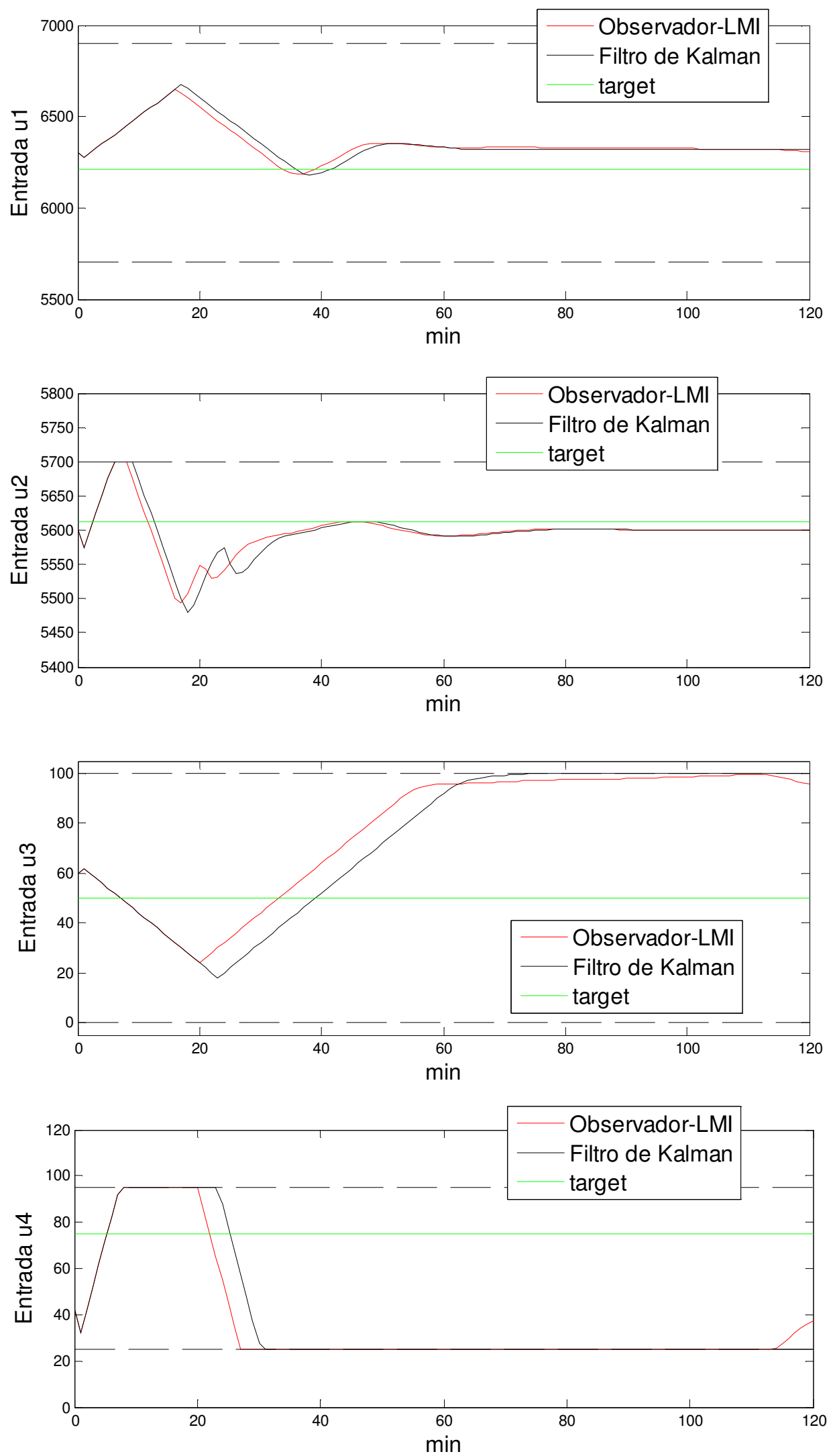

Figura 6-14 : Evolução das entradas quando o estado inicial é desconhecido 

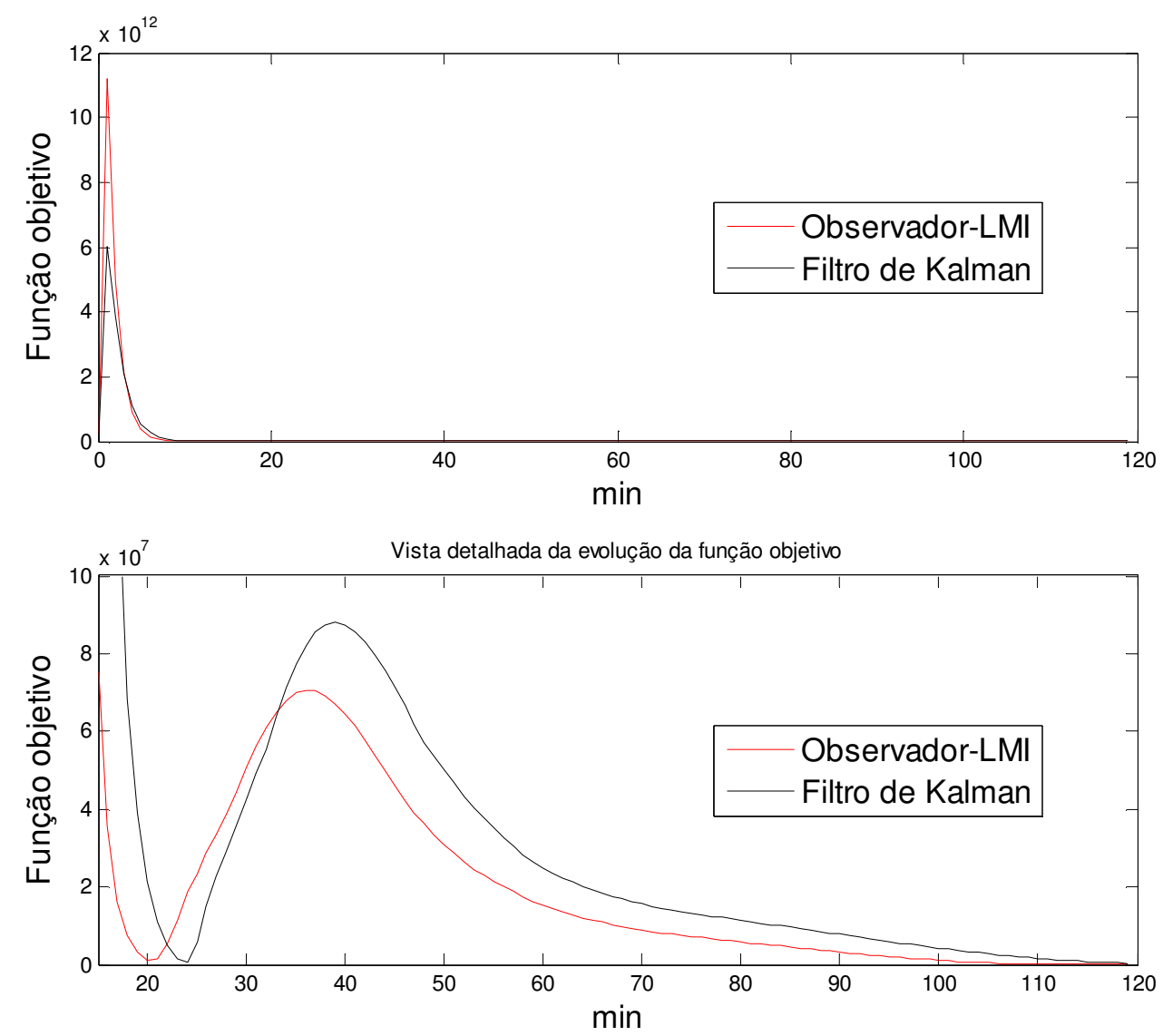

Figura 6-15 : Evolução da função objetivo quando modelo o estado inicial é desconhecido

Ao introduzir o observador heurístico, percebe-se uma melhoria com relação ao comportamento esperado das saídas $y_{1}$ e $y_{3}$. A evolução de $y_{1}$ mostra que ao utilizar esse observador sua faixa é alcançada pela primeira vez cerca de 5 minutos mais rápido do que ao empregar o Filtro de Kalman. No caso de $y_{3}$, nenhum controlador conseguiu alcançar a faixa em 120 minutos mas o baseado no observador heurístico estava cerca de $1^{\circ} \mathrm{C}$ mais próximo. Apesar de um pico inicial, a função objetivo é menor para o controlador com observador heurístico em quase todo o horizonte de simulação, indicando uma melhoria de desempenho.

\subsubsection{Caso 3: comparação entre o Filtro de Kalman e o observador heurístico com modelo incorreto}

Este Caso 3 apresenta duas fontes para a diferença entre o estado estimado e o estado real. A primeira delas é idêntica à realizada nos Casos 1 e 2, ou seja, a estimativa inicial do observador é igual a 0 apesar da planta estar em um estado estacionário em que as 
componentes $x^{s}$ são diferentes de 0 . Além disso, foi considerado que a planta evolui de acordo com constantes de tempo diferentes daquelas utilizadas pelo controlador para realizar suas predições.

Seja $\tau_{i, j, k}$ a constante de tempo do k-ésimo termo da expansão em frações parciais da função de transferência $G_{i, j}(s)$. Para o modelo do controlador, utilizaram-se os valores de $\tau_{i, j, k}$ conforme (6-1). Já para a evolução da planta, consideram-se constantes de tempo da forma

$$
\tau_{i, j, k}=\tau_{i, j, k}+\Delta \tau_{i, j, k}
$$

A incerteza $\Delta \tau_{i, j, k}$ foi gerada de modo aleatório, seguindo uma distribuição uniforme no intervalo $\left[-\tau_{i, j, k}, \tau_{i, j, k}\right]$. A sintonia utilizada nesse Caso é a apresentada na Tabela 6-8 e o horizonte de controle $m=7$ também foi mantido.

Os resultados obtidos podem ser vistos nas Figuras 6-16 a 6-18:
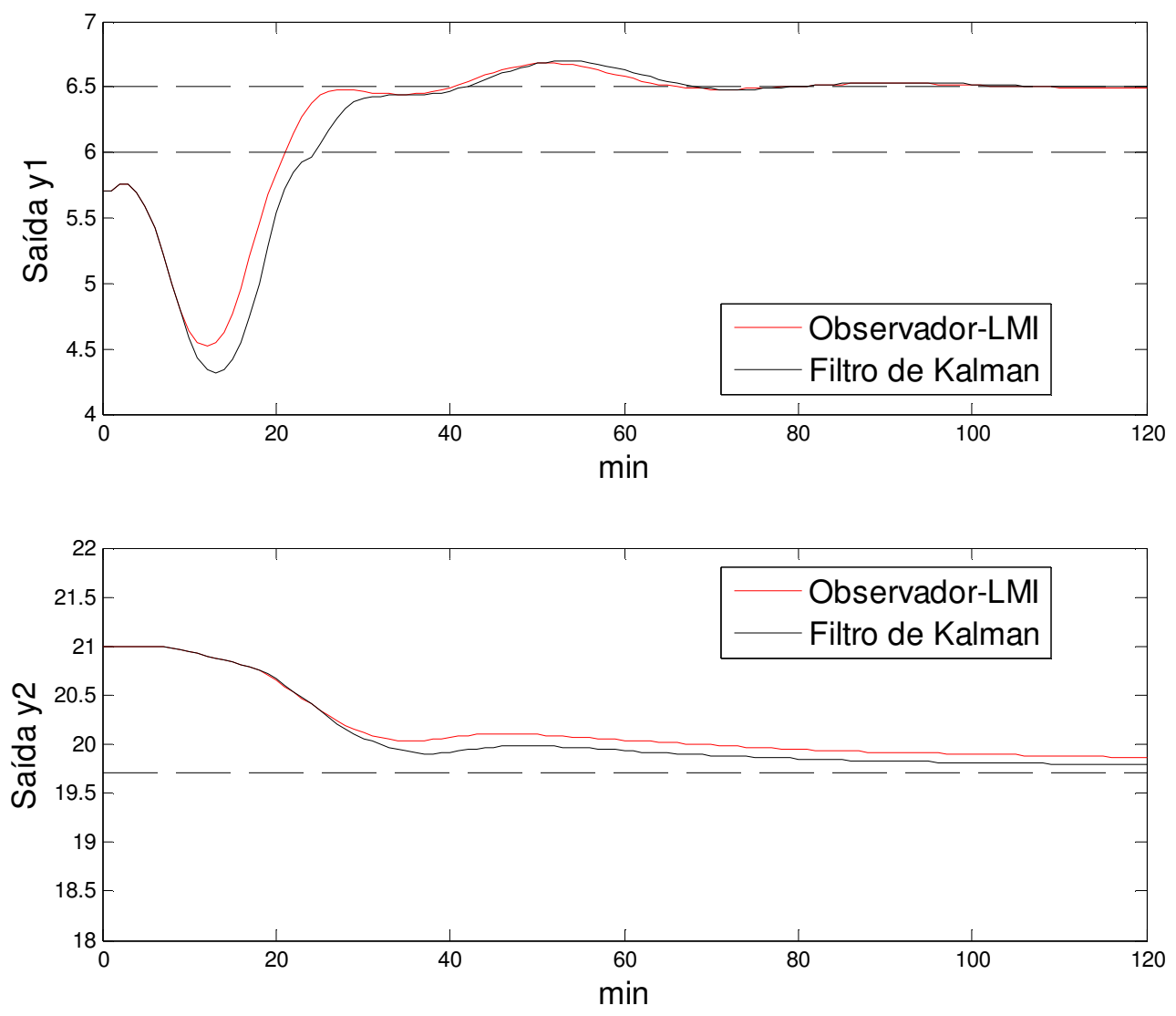

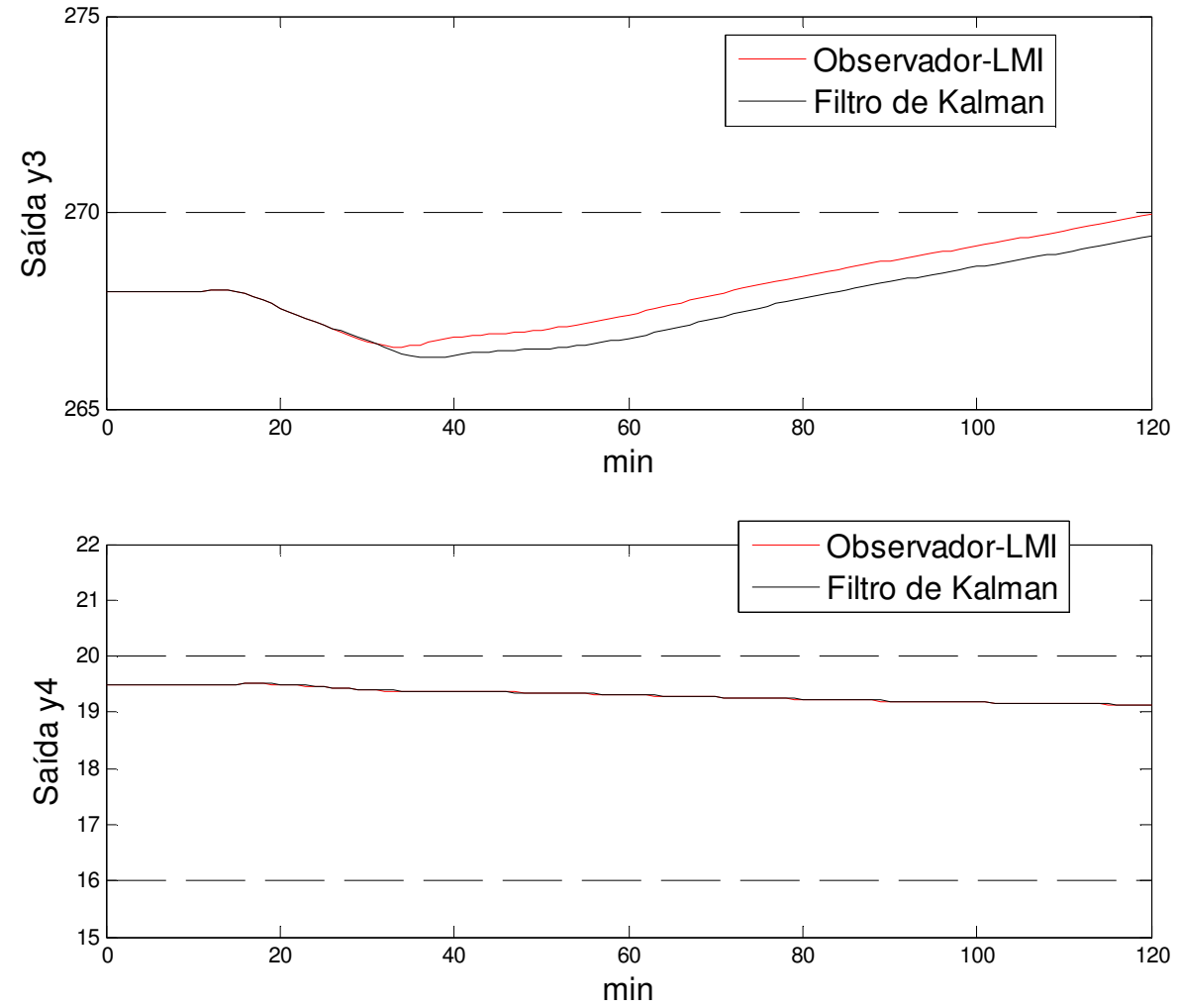

Figura 6-16 : Evolução das saídas quando modelo tem incerteza nas constantes de tempo
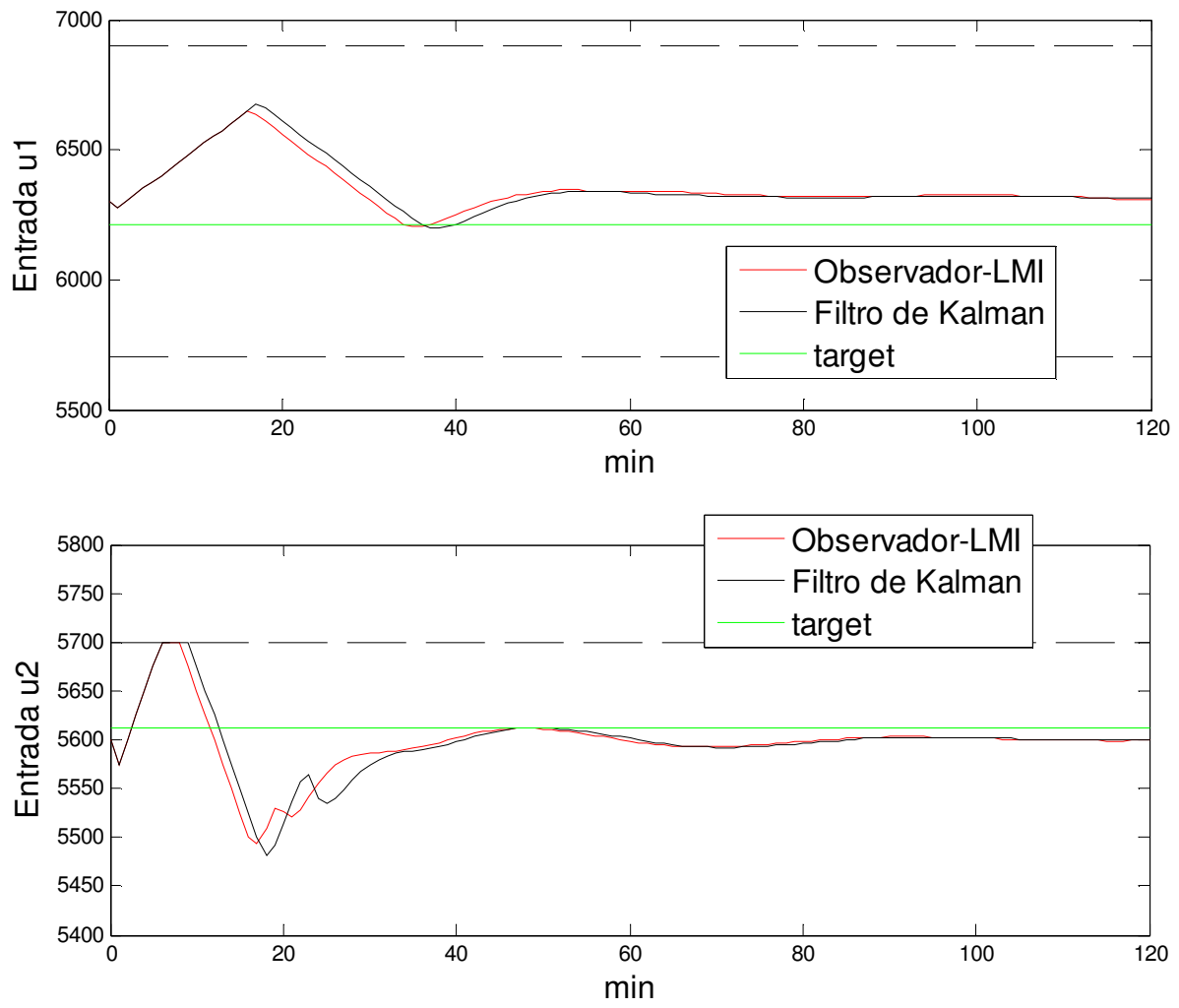

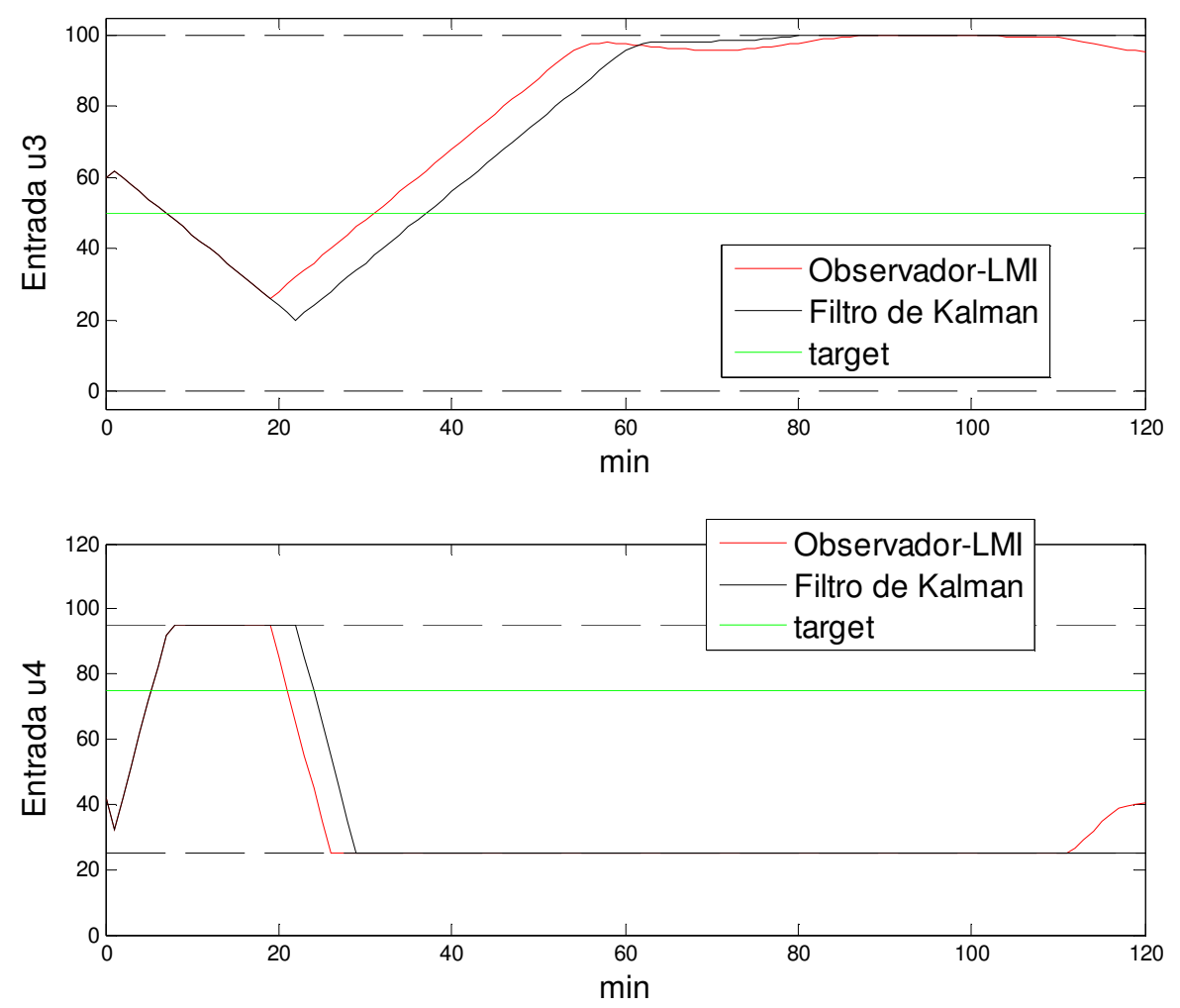

Figura 6-17 : Evolução das entradas quando modelo tem incerteza nas constantes de tempo
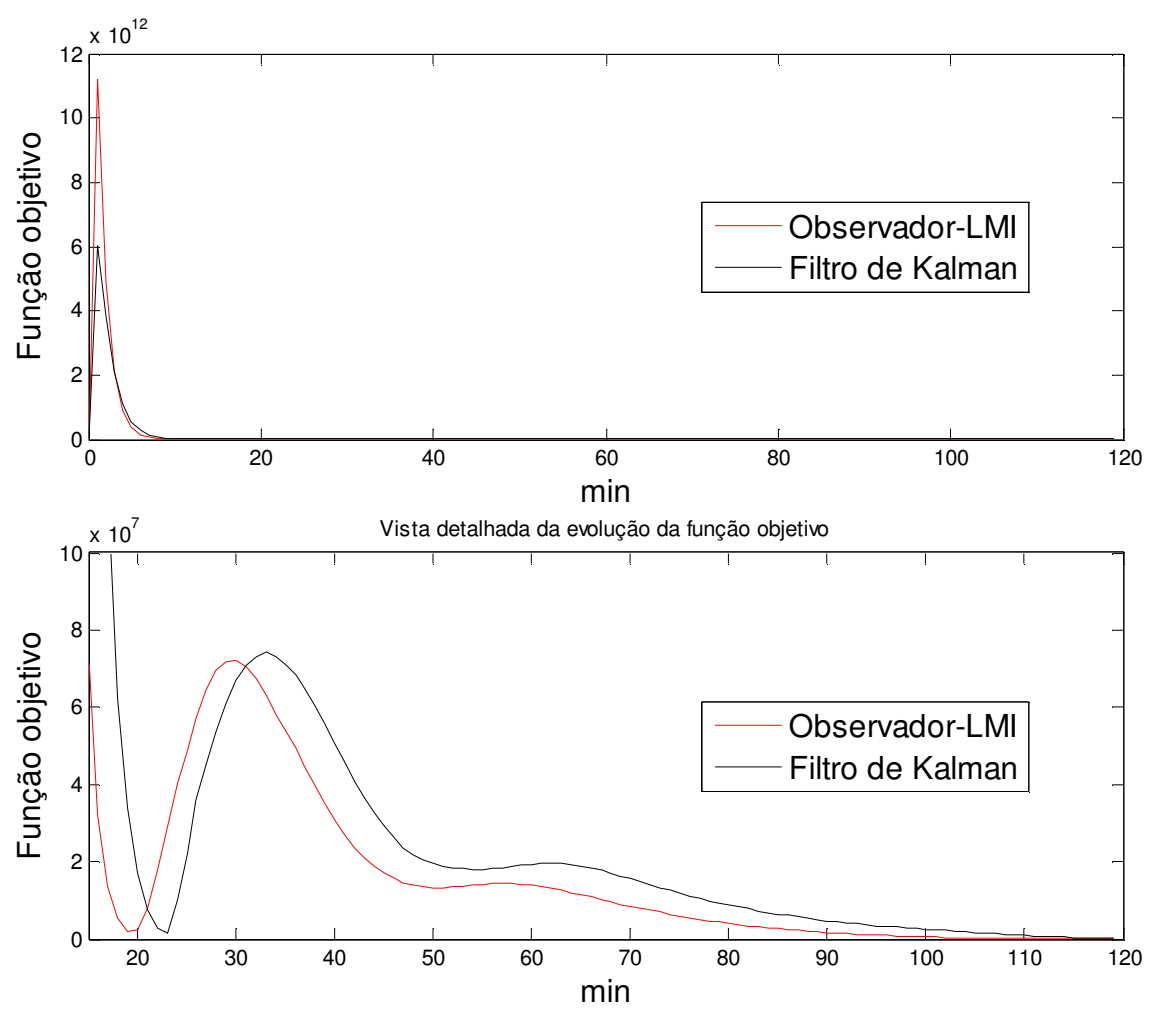

Figura 6-18 : Evolução da função objetivo quando modelo tem incerteza nas constantes de tempo 
A presença de incerteza no modelo praticamente não alterou a dinâmica do sistema, já que a evolução das entradas e saídas nos Casos 2 e 3 é praticamente idêntica. Conclui-se que o desempenho ruim dos controladores advém principalmente da incerteza com relação ao estado inicial e não do erro de modelagem representado pela incerteza nas constantes de tempo. Uma diferença no Caso 3 é a temperatura do óleo térmico ter atingido sua faixa dentro do horizonte simulado quando se empregou o controlador com observador heurístico. Com relação às funções objetivo, novamente tem-se valores mais baixos para o controlador com esse observador em quase toda a simulação. 


\section{CONSIDERAÇÕES FINAIS}

\subsection{CONCLUSÕES}

Neste trabalho foi apresentado um controlador preditivo com estabilidade nominal garantida para processos com pólos estáveis e integradores e presença de tempos mortos entre as entradas e saídas do processo. Este controlador é particularmente apropriado para processos em que haja saídas integradoras e tempos mortos diferentes entre uma saída integradora e várias entradas. Foi desenvolvido um novo modelo em variáveis de estado que representa com exatidão sistemas lineares, integradores ou não, com tempo morto, desde que o tempo morto seja um múltiplo inteiro do período de amostragem. Além disso, a realização do modelo é sempre observável, mesmo quando os tempos mortos para uma mesma entrada são diferentes, ao contrário do que acontecia com abordagens anteriores como a de Carrapiço (2004).

Outro desenvolvimento deste trabalho foi a proposição de um observador heurístico, destinado a situações em que a velocidade de convergência dos estados estimados com Filtro de Kalman não é suficientemente grande. Este observador é uma extensão da proposta inicialmente feita em Rodrigues (2001). Para as matrizes $A$ e $C$ construídas de acordo com o modelo proposto na seção 3 deste trabalho, observou-se que o algoritmo iterativo de Rodrigues (2001) não produziu observadores com dinâmicas suficientemente rápidas. Optouse por modificar aquele algoritmo, o que produz observadores mais parecidos com o Filtro de Kalman mas mais rápidos em simulações determinísticas.

O controlador proposto permite que sejam especificados valores desejados ou targets para as entradas e faixas dentro das quais se deseja manter as saídas, aumentando assim os graus de liberdade e, potencialmente, melhorando o desempenho. Essa maneira de transmitir objetivos econômicos à camada de controle supervisório não causa aumento significativo na complexidade do problema de otimização a ser resolvido, já que este continua sendo um caso de programação quadrática como é habitual na literatura de controle preditivo. Em todo caso, para evitar problemas numéricos, optou-se por realizar as simulações desse controlador utilizando um algoritmo de resolução robusto como CONOPT.

Foram desenvolvidas duas formulações para o controlador, uma mais direta (em um passo) e outra (dois passos) com a qual se pode provar a estabilidade nominal do controlador. As simulações apresentadas neste trabalho indicam que a performance tende a ser melhor no controlador em um passo, com as saídas retornando às suas faixas em menos tempo. No 
entanto, desde que haja uma sintonia específica para o controlador em dois passos, sua performance também pode ser suficientemente boa. No caso de uma aplicação real, possivelmente seria preferível utilizar a formulação em um passo, apesar da falta de garantia formal de estabilidade.

Através de algumas simulações, foi comparado o desempenho do observador proposto em relação ao Filtro de Kalman. Pode-se perceber que, especialmente para a concentração de oxigênio no reator, a utilização do observador heurístico é mais recomendada, já que neste caso essa variável controlada tende a se aproximar mais rapidamente de sua zona préespecificada. Contudo, quando os sinais ou processos da planta estiverem sujeitos a níveis muito intensos de ruídos ou perturbações não medidas, a maior agressividade do controlador quando acoplado ao observador heurístico pode levar à instabilidade da planta.

\subsection{SUGESTÕES DE CONTINUIDADE}

O controlador proposto neste trabalho apresenta apenas estabilidade nominal. Sabendo-se que todo modelo apresenta, na prática, algum desvio em relação ao sistema real que ele busca representar, é necessário saber como seria o comportamento deste controlador quando suas predições diferirem da dinâmica real. Logo, uma extensão possível seria a formulação do controlador em um contexto multi-plantas, ou seja, admitindo-se que o comportamento exato da planta seja desconhecido mas restrito a um politopo determinado por um conjunto de modelos típicos. Nessa situação mais geral, poderia-se mostrar a estabilidade robusta do controlador, de maneira semelhante ao que foi proposto em González, Marchetti e Odloak (2007).

A escolha do observador de estados pode influenciar significativamente o comportamento do sistema em malha fechada, conforme ilustrado na seção 6.3. Por isso, algum esforço pode ser direcionado para a melhoria do observador heurístico proposto. Como o cálculo do ganho do observador heurístico proposto é obtido através da resolução de um problema de otimização em um domínio não-convexo, é possível que a solução obtida seja sub-ótima.

Métodos mais sofisticados de resolução de problemas do tipo BMI são sugeridos em VanAntwerp e Braatz (2000), incluindo, por exemplo, técnicas que permitam a obtenção de valores ótimos globais, como branch and bound. Essencialmente, algoritmos desse tipo também obtêm a solução de uma BMI através da resolução repetida de LMIs. Ao contrário do algoritmo de Rodrigues (2001), contudo, as LMIs auxiliares podem ser formuladas de 
maneira a serem relaxações mais próximas do problema real, permitindo assim um tratamento numérico relativamente fácil mas sem se afastar demais do problema não-convexo original.

Uma alternativa para o desenvolvimento de observadores mais adequados seria a utilização de técnicas de síntese de filtros robustos, em que a representação prévia das incertezas a que o modelo está sujeito permite calcular um ganho do observador que atenda a critérios de performance e de estabilidade, como descrito em Mangoubi (1998). 


\section{REFERÊNCIAS}

BOYD, S.P., GHAOUI, L. E., FERON, E. \& BALAKRISHNAN, V. Linear matrix inequalities in system and control theory, Philadelphia: SIAM, 1994

CARRAPIÇO, O. L., ODLOAK, D. A stable model predictive control for integrating processes. Computers and Chemical Engineering, v. 29, n. 5, p. 1089-1099, 2005.

CARRAPIÇO, O. L. Controle Preditivo de Horizonte Infinito para Processos Integradores com Tempo Morto. 2004. 102 p. Dissertação de mestrado. Escola Politécnica, Universidade de São Paulo, São Paulo, 2004.

CAMACHO, E. F.; BORDONS, C. Control predictivo: pasado, presente y futuro. Revista Iberoamericana de Automática e Informática Industrial, v.1, n. 3, p. 5-28, 2004.

CUTLER, C. R.; RAMAKER, B. L. Dynamic matrix control - a computer control algorithm. Proceedings Joint Automatic Control Conference, San Francisco, CA (EUA). 1980.

GARCIA, C. E., PRETT, D. M.; MORARI, M. Model predictive control: theory and practice-a survey. Automatica, v. 25, n. 3, 335-348, 1989.

GONZÁLEZ, A. H., MARCHETTI, J. L.; ODLOAK, D. Extended robust model predictive control of integrating systems. American Institute of Chemical Engineering Journal, v. 53, n. 7, p. 1758-1769, 2007.

GONZÁlEZ, A. H.; ODLOAK, D. A Stable MPC with zone control. Journal of Process Control, v. 19, n. 1, p. 110-122, 2009

GLAD, T.; LJUNG, L. Control Theory. London: Taylor \& Francis, 2000.

HESPANHA, J. P. Linear Systems Theory. New Jersey: Princeton University Press, 2009. 
KEERTHI, S. S.; GILBERT, E. G. Optimal infinite horizon feedback laws for a general class of constrained discrete time systems: Stability and moving-horizon approximations. Journal of Optimization Theory and Application, v. 57, p. 265-293.

LEE, J. H.; XIAO, J. Use of two-stage optimization in model predictive control of stable and integrating systems. Computers and Chemical Engineering, v. 24, n. 2-7, p. 1591-1596, 2000 .

LEE, J. H., MORARI, M.; GARCIA, C. E. State-space interpretation of model predictive control. Automatica, v. 30, n. 4, p. 707-717, 1994.

MORARI, M.; LEE, J. H. Model predictive control: past, present and future. Computers \& Chemical Engineering, v. 23, n. 4-5, p. 667-682, 1999.

MACIEJOWSKI, J.M. Predictive Control with Constraints. New Jersey: Prentice Hall, 2002.

MANGOUBI, R. S. Robust Estimation and Failure Detection. London: Springer, 1998.

MICHALSKA, H.; MAYNE, D. Q. Robust receding horizon control of constrained nonlinear systems. IEEE Transactions on Automatic Control, v. 38, n. 11, p. 1623-1633, 1993.

MUSKE, K. R.; RAWLINGS, J. B. Model predictive control with linear models. American Institute of Chemical Engineering Journal, v. 39, n. 2, p. 262-287, 1993.

RAWLINGS, J. B.; MUSKE, K. R. The stability of constrained receding horizon control. IEEE Transactions on Automatic Control, v. 38, n. 10, p. 1512-1516, 1993.

RICHALET, J., RAULT, A., TESTUD, J. L.; PAPON, J. Model predictive heuristic control: Applications to industrial processes. Automatica, v.14, p.413-428, 1978.

RODRIGUES, M. A. Estabilidade robusta de controladores preditivos. 2001. 147 p. Tese de doutorado, Escola Politécnica, Universidade de São Paulo, São Paulo, 2001. 
RODRIGUES, M. A.; ODLOAK D. An infinite horizon model predictive control for stable and integrating processes. Computers Chemical Engineering, v.27, n. 8-9, p. 1113-1128, 2003.

SIMON, D. Optimal State Estimation. New Jersey: John Wiley \& Sons. 2006.

VANANTWERP, J. G.; BRAATZ, R. D. A tutorial on linear and bilinear matrix inequalities. Journal of Process Control, v. 10, p. 363-385, 2000.

ZANIN, A.C. TVRZSKA DE GOUVÊA, M.; ODLOAK, D. Integrating real time optimization into the model predictive controller of the FCC system, Control Engineering Practice, v. 10, n. 8, p. 819-831, 2002

ZANIN, A. C. Implementação industrial de um otimizador em tempo real. 2001. 161 p. Tese de doutorado, Escola Politécnica, Universidade de São Paulo, São Paulo, 2001. 